\title{
Silicon-On-Insulator Biosensor using Micro-ring Resonators and Ring-assisted Mach-Zehnder Interferometers
}

\author{
by
}

Owen Marsh

A thesis submitted to the Faculty of Graduate and Postdoctoral Affairs in partial fulfillment of the requirements for the degree of

\author{
Masters of Applied Science \\ in \\ Electrical and Computer Engineering \\ Carleton University \\ Ottawa, Ontario \\ (C) 2016, Owen Marsh
}




\begin{abstract}
The silicon photonic platform is widely used for optical sensing and communications because its high index contrast allows for smaller devices, and is compatible with CMOS manufacturing techniques. In this thesis we examine theoretically and demonstrate experimentally silicon-on-insulator biochemical sensors based on micro-ring resonators (MRR) and ring assisted Mach-Zehnder interferometers (RAMZI). We determine that the sensitivity of the slot waveguide is enhanced compared to the wire waveguide and examine the effect of coupler design on sensing performance using directional couplers and multi mode interference (MMI) couplers. We find that RAMZI devices offer a similar sensitivity while offering a larger index of refraction measurement range than MRR designs. The slot waveguide is found to increase bulk index sensing sensitivity by up to 2.2 times that of the wire waveguide. Directional coupler based MRR and RAMZI devices are shown to have higher bulk index sensitivities than MMI coupled devices.
\end{abstract}




\section{Acknowledgements}

I would like to acknowledge the help and contributions of all the people that made this thesis possible. First I would like to thank Yule Xiong, for his previous work on this project and giving me a basis for my work. I would like to thank Angela McCormick for her assistance in etching the chips for testing. Also I would like to thank Rob Vandusen with his help setting up vibration isolators so that I could perform the testing. I would like to thank Ian Marsh in his assistance in editing the spelling and grammar of my thesis. Finally I would like to express my sincere thanks to my supervisor Dr. Winnie Ye, for her advice, motivation and her time spent revising and editing my thesis. 


\section{Table of Contents}

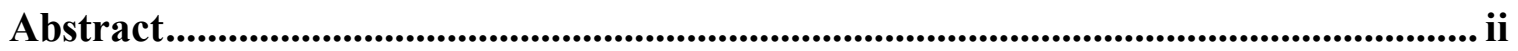

Acknowledgements .............................................................................................................................ii

Table of Contents ....................................................................................................... iv

List of Tables ................................................................................................................................ vi

List of Illustrations...................................................................................................... vii

1 Chapter: Introduction ........................................................................................................ 1

1.1 Thesis Objectives and Organization................................................................... 4

2 Chapter: Literature Review................................................................................... 5

3 Chapter: Theory and Concepts ............................................................................. 13

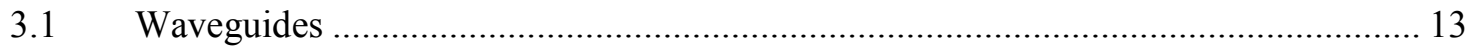

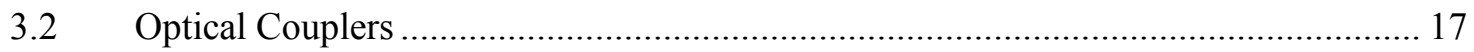

3.2.1 Directional Coupler ........................................................................................ 17

3.2.2 Multimode Interference Coupler ...................................................................... 19

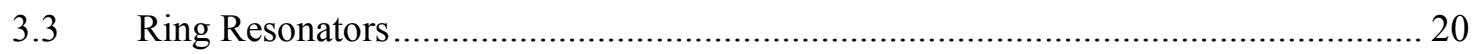

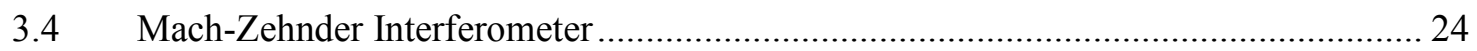

3.5 Ring Assisted Mach-Zehnder Interferometer ............................................................ 26

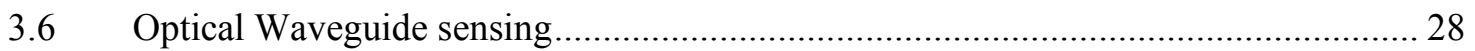

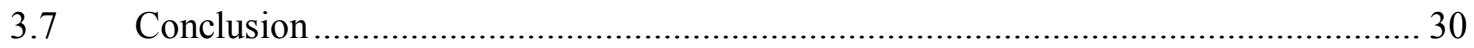

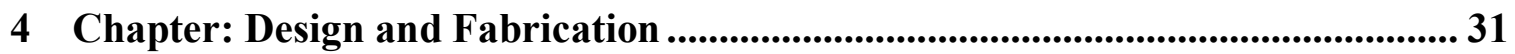

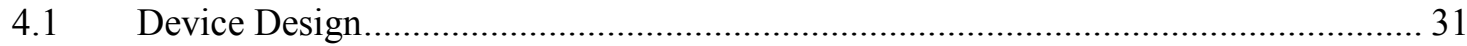

4.2 Fabrication Tolerance Simulations ………………….......................................... 34

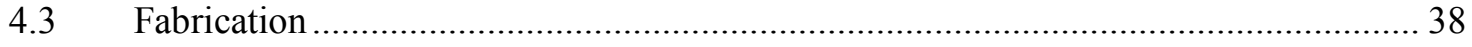

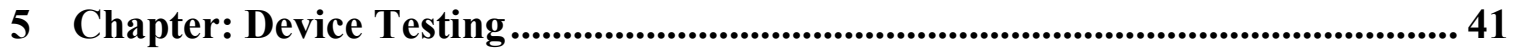




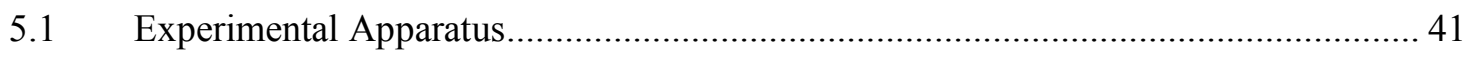

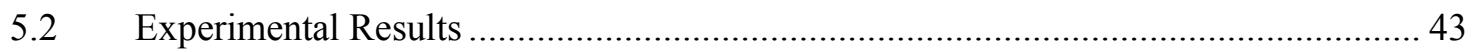

5.2.1 Devices Characteristics - DC- and MMI-based devices using wire waveguides .... 45

5.2.2 Devices Characteristics - DC- and MMI-based devices using slot waveguides ..... 50

5.2.3 Bulk Index Sensing using DC- and MMI-coupled wire waveguides ..................... 56

5.2.4 Bulk Index Sensing using DC- and MMI-coupled slot waveguides .......................59

6 Chapter: Conclusion .......................................................................................................... 65

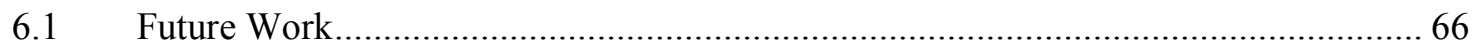

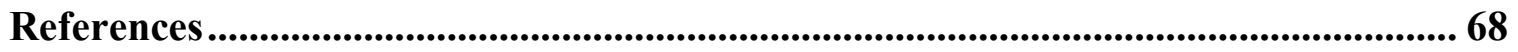




\section{List of Tables}

This is the List of Tables.

Table 1: Simulated Coupling coefficient for the DC with wire waveguides and slot

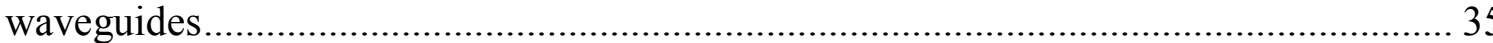

Table 2: Simulated coupling coefficient for the MMI coupler with sire waveguides ...... 37

Table 3: Simulated coupling coefficient for the MMI coupler with slot waveguides ...... 37

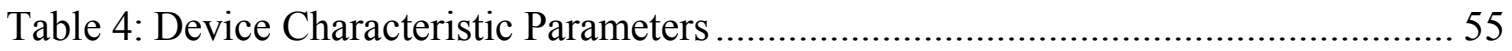

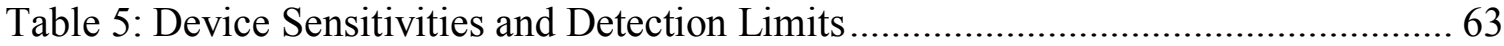




\section{List of Illustrations}

Figure 1: Right, microdisk based optical chemical sensors as demonstrated by E.

Krioukov et al. [39] Left, MRR based optical chemical sensors as demonstrated by A.

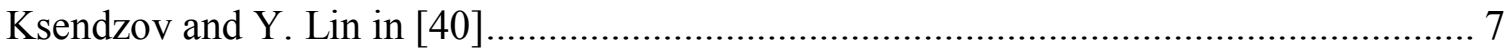

Figure 2: Right, SOI MRR optical biosensor demonstrated by Vos et al. in [43] Left, (a) regular MRR based optical sensors, (b) Folded cavity MRR optical cavity senor as

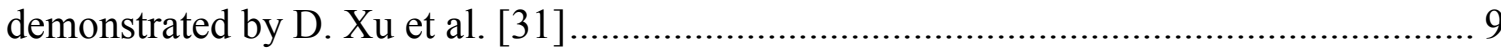

Figure 3: Left, slot based MRR chemical sensors demonstrated by T. Claes et al.[28]

Right, RAMZI based chemical sensor demonstrated by D. Dai in [33] .......................... 11

Figure 4: Schematic drawing for the four common waveguide designs........................... 13

Figure 5: Schematic of a Slab waveguide ................................................................... 14

Figure 6: Schematic of the Directional Coupler ........................................................ 18

Figure 7: Left, Schematic of the MMI coupler. Right. Simulation of the MMI coupler. . 20

Figure 8: A) single coupler design, B) double coupler design [55] ................................ 21

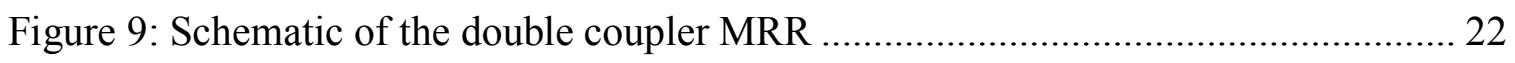

Figure 10: Schematic of the MZI device[49] ............................................................. 25

Figure 11: Schematic of ring-assisted MZI (RAMZI) racetrack design. The couplers can be directional couplers (DCs) or multimode interference (MMI) couplers. $\backslash \ldots \ldots \ldots \ldots \ldots \ldots \ldots . . . .26$ Figure 12: Cross section of the wire waveguide and slot waveguide designs implemented.

Figure 13: FDTD Simulated power cross coupling ratios (blue) and power coupling loss (red) as a function of wavelength for (a) DC coupler and (b) MMI coupler 32

Figure 14: MRR and RAMZI wire waveguide sensor designs; all with a radius of $6 \mu m 33$ 
Figure 15: MRR and RAMZI slot waveguide sensor designs with a radius of $20 \boldsymbol{\mu m} \ldots . . .34$ Figure 19: Simulated Electric field profiles; A) Electric field profile for the wire waveguide B) Electric Field profile for the Slot Waveguide C) Cross section of the electric field profile for the wire waveguide along $\mathrm{z}=0.11 \mathrm{~nm} \mathrm{D)} \mathrm{Cross} \mathrm{section} \mathrm{of} \mathrm{the}$

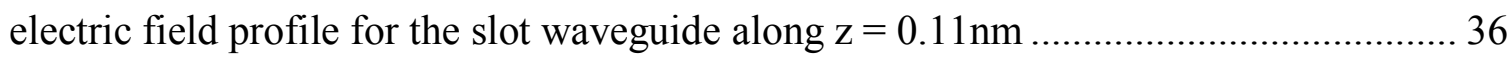
Figure 16: Fabricated MRR and RAMZI wire waveguide sensors; all with a radius of

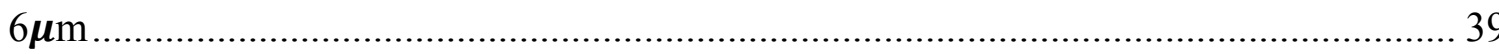

Figure 17: Fabricated MRR and RAMZI slot waveguide sensors with a radius of $20 \mu \mathrm{m}$,

Figure 18: Left: OZ optics tapered and lensed optical Fiber[60], Right: Inverse nanotaper coupler design[61] 40

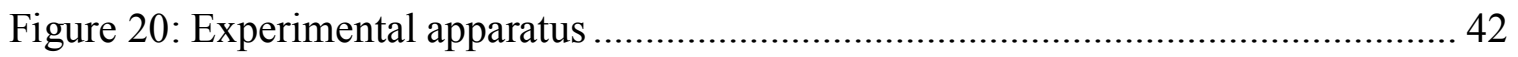

Figure 21: Transmission spectrum and transmission of a single peak for a DC-coupled MRR with wire waveguides.

Figure 22: Transmission spectrum and transmission of a single peak for the MMI-coupled MRR with wire waveguides. 47

Figure 23: Transmission spectrum and transmission of a single peak for the DC-coupled RAMZI device with wire waveguides. The difference in arm lengths is $\boldsymbol{\Delta} \boldsymbol{L}=12.05 \boldsymbol{\mu m} .48$ Figure 24: Transmission spectrum and transmission of a single peak for the MMI-coupled RAMZI device with wire waveguides. The difference in arm length is $\boldsymbol{\Delta} \boldsymbol{L}=13.14 \boldsymbol{\mu} \mathrm{m} \ldots .49$ Figure 25: Transmission spectrum and transmission of a single peak for the DC-coupled MRR device with slot waveguides 51 
Figure 26: Transmission spectrum and transmission of a single peak for the MMI-coupled MRR device with slot waveguides. 51

Figure 27: Transmission spectrum and transmission of a single peak for the DC -coupled RAMZI device with slot waveguide with an arm length difference of $\boldsymbol{\Delta L}=13.08 \boldsymbol{\mu m} \ldots 52$ Figure 28: Transmission spectrum and transmission of a single peak for the MMI-coupled RAMZI with slot waveguides. The arm length difference of $\boldsymbol{\Delta} \boldsymbol{L}=13.14 \boldsymbol{\mu m}$ 53

Figure 29: Transmission of One Peak for various sugar concentrations and the Change of resonant peak location vs sugar concentration for the DC-coupled MRR with wire waveguides. 57

Figure 30: Transmission of One Peak for various sugar concentrations and the Change of resonant peak location vs sugar concentration for the MMI-coupled MRR with wire waveguides.

Figure 31: Transmission of One Peak for various sugar concentrations and the Change of resonant peak location vs. sugar concentration for the DC-coupled RAMZI with wire waveguides . The difference in arm length is $\boldsymbol{\Delta L}=12.05 \boldsymbol{\mu m}$. 58 Figure 32: Transmission of One Peak for various sugar concentrations and the Change of resonant peak location vs. sugar concentration for the MMI-coupled RAMZI with wire waveguides. The difference in arm length is $\boldsymbol{\Delta} \boldsymbol{L}=13.14 \boldsymbol{\mu m}$ 59 Figure 33: Transmission of One Peak for various sugar concentrations and the Change of resonant peak location vs. sugar concentration for the DC-coupled MRR with slot waveguides. 60 
Figure 34: Transmission of One Peak for various sugar concentrations and the Change of resonant peak location vs. sugar concentration for the MMI-coupled MRR with slot waveguides. 60

Figure 35: Transmission of One Peak for various sugar concentrations and the Change of resonant peak location vs sugar concentration for the DC-coupled RAMZI with slot waveguides. The difference in arm length is $\Delta \boldsymbol{L}=13.08 \mu \boldsymbol{m}$ 62 Figure 36: Transmission of One Peak for various sugar concentrations and the Change of resonant peak location vs sugar concentration for the MMI-coupled RAMZI with slot waveguides. The difference in arm length is $\Delta \boldsymbol{L}=13.14 \mu \mathrm{m}$ 62 


\section{List of Abbreviations}

- ER: extinction ratio

- RIU: refractive index units

- MRR: micro-ring resonator

- RAMZI: ring assisted Mach-Zehnder interferometer

- SOI: silicon-on-insulator

- DC: directional coupler

- MMI: multimode interference

- FWHM: full width half maximum

- Q-factor: quality factor

- FSR: free spectral range

- MZI: Mach-Zehnder interferometer 


\section{Chapter: Introduction}

In recent years photonic devices have become fundamental components in a wide range of fields from the optical routers that are the backbone of the internet[1], to laser wielding systems which are found in widespread use in automotive and other manufacturing industries [2], and many other fields around the globe. Sensing is another common application in the photonics field with a wide variety of designs and uses; from biological and chemical sensors, to sensors to detect structural flaws in materials [3]. With global market value of $\$ 315.9$ million in 2015 and a projected to growth to $\$ 971.8$ million by 2020 [4] photonic sensing is a promising field for research and development.

Since the early years of photonics in the 1960s, an important goal research and technical development has been the realization of large scale optical integrated circuit [5]. The dominance of silicon based integrated circuits in the electronics industry led to the investigation of silicon photonic circuits in the early 1980s [6], [7], [8] The silicon platform, specifically the high refractive index contrast of the Silicon-on-Insulator (SOI) platform, offers high optical confinement, allowing for micrometer and nanometer sized device footprints. In addition, SOI's compatibility with the mature CMOS fabrication techniques from the microelectronics industry offer great potential for low cost mass production of high density photonic integrated circuits. The SOI platform is highly appealing to researchers and has attracted a large amount of research and development activity in recent years. Numerous active and passive devices have been demonstrated, such as low loss waveguides [9], optical filters [10], [11], modulators [12], [13], detectors [14], [15] and most recently silicon based Raman lasers [16], [17]. 
Optical based sensors were first demonstrated in late 1980s [18] based on grating couplers and interferometers. Since then much work has been done developing a wide variety designs for a broad variety of applications. Many devices have been designed for biological and chemical sensing, and this subject is of interest for this thesis. The overarching goal of all work on biological and chemical sensors is the realization of a fully integrated sensing system, known as a 'lab-on-a-chip'. A lab-on-a-chip system contains multiple sensing elements and micro-fluidic channels to control the flow of reagents into reaction chambers, allowing for controlled reactions, with the sensor arrays measuring the outputs. Advantages of lab-on-a-chip implementations include low reagent volumes, quicker analysis and production of results, more precise process control, and lower cost of testing. [19], [20]

The majority of the chemical and biological sensors that have been developed are based on evanescent field sensing, in which a waveguide is coated with a layer of a chemically sensitive material which interacts with specific chemicals or biomolecules. The changed surface chemistry alters how the light passes through the waveguide by either changing the phase or absorption of the light through the waveguide [21], [22]. The waveguide can be integrated as an arm of a interferometer, a ring resonator, Bragg grating, or an input or output coupler [23]-[26].

Recently biochemical sensors based on micro-ring resonators (MRRs) built on the SOI platform have been demonstrated with both wire waveguides and slot waveguides [27]-[29]. Q-factor is a measure of sharpness of the resonant peaks relative to the resonant wavelength. The high Q-factor of these devices allows for high sensitivity and low detection limit sensing with micron scale size devices. The slot waveguides devices 
have been shown to increase the sensitivity of a factor of 3.5 when compared to the conventional wire waveguide design [28]. Most designs implemented use a directional coupler due to its lower optical loss. This produces higher Q-factor devices. However the directional coupler is highly susceptible to variations in fabrication and has low bandwidth. Compared to directional couplers, the performance of a multimode interferometer coupler is less sensitive to variations in fabrication and has higher bandwidth [30]. However the multimode interferometers suffer higher losses that result in lower Q-factors in micro-ring resonators.

The simplest method for increasing the $\mathrm{Q}$ factor of a MRR is to increase its radius [31]. However the increase in radius produces a longer optical path which causes a decrease in the free spectral range (free spectral range (FSR) is the distance between adjacent resonant peaks). The decrease in the FSR limits the range of distinguishable measurements. Recently, a unique design was proposed [32] and demonstrated [33] by integrating a Mach-Zehnder Interferometer with a MRR, referred to as a Ring Assisted Mach-Zehnder Interferometer (RAMZI). The RAMZI device can be designed in such a way that a large quasi-FSR can be obtained from dominant resonant peaks with large extinction ratios. It was also shown that the device increases the overall sensitivity of the MRR.

Integrating the slot waveguide with the RAMZI device would result in a device with both a high sensitivity and a high sensing range, making it an improved device for a variety of sensing purposes. The effects of the coupler design on the sensing with microring resonator and RAMZI devices has not been fully investigated. Knowledge resulting 
from such an investigation could allow for improved sensor designs, featuring both high measurement sensitivity and lower susceptibility to fabrication errors.

\subsection{Thesis Objectives and Organization}

The goal of this thesis is to examine and demonstrate MRRs and RAMZI based biochemical sensors. We will examine the effect of coupler design on sensing performance, using directional couplers and multimode interference couplers. We will also examine the sensitivity enhancement of the slot waveguide when compared to the wire waveguide. Finally we will compare all of the designs and determine the optimal design for biological and chemical sensors.

Chapter 2 is a review of past work done into the field of photonic sensors and on the silicon-on-insulator platform regarding its use as biological and chemical sensors. In Chapter 2 MRR and RAMZI designs are introduced.

Chapter 3 reviews the theory and physics of optical waveguides and the advantages and disadvantages of coupler designs that are being investigated. The theory of the MRRs and the RAMZI device design are also discussed as well as the theory of optical based sensing. The theoretical sensitivities of the MRR and RAMZI devices are presented.

Chapter 4 discusses the device designs and the geometries of the waveguide designs, coupler designs and the MRR and RAMZI devices. It examines the design choices and limitations of the fabrication services as well as the required additions to the devices to allow for the testing. The chapter finishes with an overview of the fabricated devices. 
Chapter 5 discusses the experimental setup and the experimental results obtained from testing our devices. The experimental results are separated into two sections; first we will examine the characteristics of each devices, and second, the bulk index sensing of each device. The bulk index sensing was performed using a sucrose solution of varying concentrations; the concentration of sugar linearly shifts the index of refractions. The change in the index of refraction causes a shift in the peak location which can be tracked to determine the characteristics of the devices.

Finally, Chapter 6 reviews the present work and achievements and outlines ideas for future work to increase the performance of our sensor further the pursuit into lab-ona-chip sensors.

\section{Chapter: Literature Review}

This chapter includes a literature review on biological and chemical photonic sensors, with a focus on the silicon-on-insulator micro-ring resonator, and the ring assisted Mach-Zehnder interferometer.

Descriptions of photonic bio-chemical sensors were first published in the late 1980s [34]. The early devices were primarily based on grating coupler and interferometers designs. The mechanism of operation of the devices was the variation of the index of refraction, causing a variation in the input and output coupling angles and a phase change in the guided modes for the two device designs respectively. These early devices were based on $\mathrm{SiO}_{2}-\mathrm{TiO}_{2}$ waveguides operating at $632.8 \mathrm{~nm}$ wavelength with the devices having a minimum resolution of $\Delta N=2 \times 10^{-6}$ and $\Delta N=0.8 \times 10^{-7}$ for the grating coupler and interferometer based designs respectively. These early devices 
showed the promise of the field and demonstrated the increased capability of the interferometer based devices.

In the following years sensors based on grating couplers, difference, MachZehnder, and Young interferometers [35]-[38] were proposed and experimentally demonstrated. A disadvantage of these devices was the requirement for large sampling areas. This led to investigation into micro cavity based sensors. E. Krioukov et al. [39] first demonstrated a micro cavity based bio-chemical optical sensor. They implemented a silicon nitride micro-disk with a radius of $15 \mu \mathrm{m}$, and a height of $255 \mathrm{~nm}$. With the design they were able to achieve a detection limit on the order of $10^{-4}$ RIU however they indicated that with optimization a detection limit of $10^{-9}$ RIU could potentially be achieved. This early demonstration of a micro cavity sensor indicated the potential that micro cavity based sensors could be as sensitive as interferometer and grating coupler devices while significantly reducing the sensor size.

The utility of micro cavity based designs are limited by the complex modal structure of the micro cavity. The micro ring resonator is conceptually similar but due to the ability to design a single mode ring it eases the data collection and allows for high sensitivity and a high dynamic range. A. Ksendzov and Y. Lin where the first to demonstrate a ring resonator based sensor [40]. The design implemented by Ksendzov and $\mathrm{Lin}$ used $\mathrm{Si}_{\mathrm{x}} \mathrm{N}_{\mathrm{y}} / \mathrm{SiO}_{2}$ waveguides with ring radius of $2 \mathrm{~mm}$ with directional couplers. The devices were used as a biochemical sensor to measure surface binding of the protein avidin to a biotin surface layer. Using their device they were able to sense $0.3 \mathrm{nM}$ of avidin in a saline solution and they calculated a detection limit of $0.1 \mathrm{nM}$, which they 
claim to compare favorably with the other optical protein detection techniques of the time.
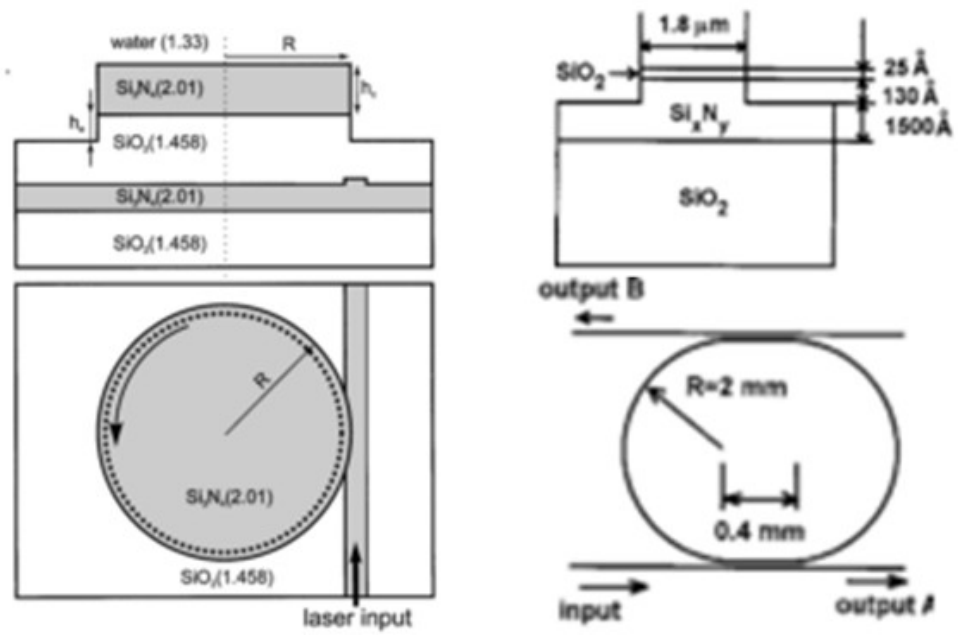

Figure 1: Right, microdisk based optical chemical sensors as demonstrated by E. Krioukov et al.

[39] Left, MRR based optical chemical sensors as demonstrated by A. Ksendzov and Y. Lin in [40]

Densmore et al. [27] demonstrated an evanescent field sensor based on the SOI platform. They theorize that the SOI platform would be ideal for chemical and biological sensing applications due to the high index contrast (HIC) between core and cladding [18], [41], [42]. The HIC allows the evanescent tails to be large and highly localized. This gives an increase in the interaction between the evanescent field and the sensing medium. The device experimentally demonstrated by Densmore et al. is a single mode SOI waveguide with a rectangular cross section of $0.26 \mathrm{um} \times 0.45 \mathrm{um}$. This waveguide is used as an exposed arm of a MZI device. This arrangement demonstrated a homogeneous sensing sensitivity of $\delta N_{e f f} / \delta N_{c}=0.31$ for a aqueous solution with a $n_{c} \sim 1.32$. The authors predicted that due to the compatibility with existing surface functionalization 
chemistries used to attach anti-bodies and DNA receptor molecules the device would be well suited for biological and chemical sensing.

The first SOI based micro ring resonator for sensing was demonstrated by K. De Vos et al. [43]. De Vos et al. implemented a micro ring with a $5 \mu \mathrm{m}$ radius and used DC couplers with a rectangular waveguide with a cross section of $500 \mathrm{~nm} \times 220 \mathrm{~nm}$. They demonstrated the device as both a bulk index sensor and a protein interaction based sensor. De Vos et al. tested their device as a bulk index sensor using a sodium chloride solution with varying concentrations. They achieved a sensitivity of $70 \mathrm{~nm} / \mathrm{RIU}$ and determined a minimal detectable refractive index shift of $10^{-5}$ RIU. They also tested the device as surface sensor and were demonstrated a detection limit of $10 \mathrm{ng} / \mathrm{ml}$ of avidin in a buffered solution binding to a biotin surface coating. This was an early demonstration of the capabilities of the SOI micro ring as a biochemical sensor.

D. Xu et al. demonstrated a similar design [31]. They created a micro ring resonator using waveguide design implemented in [27] and determined that a large radius offers the same sensitivity while reducing the dependence of the device on waveguide loss or coupling variations. In order reduce the size of the device they folded the cavity, producing a device with $1.27 \mathrm{~mm}$ of sensor interaction length to a $110 \mu \mathrm{m} \times 110 \mu \mathrm{m}$ area. With this design they were able to sense binding events of a $1.7 \mathrm{nM}$ streptavidin binding to biotin. The $\mathrm{D}$. Xu et al. device demonstrated a step forward toward lab-on-a-chip molecular sensor arrays. 

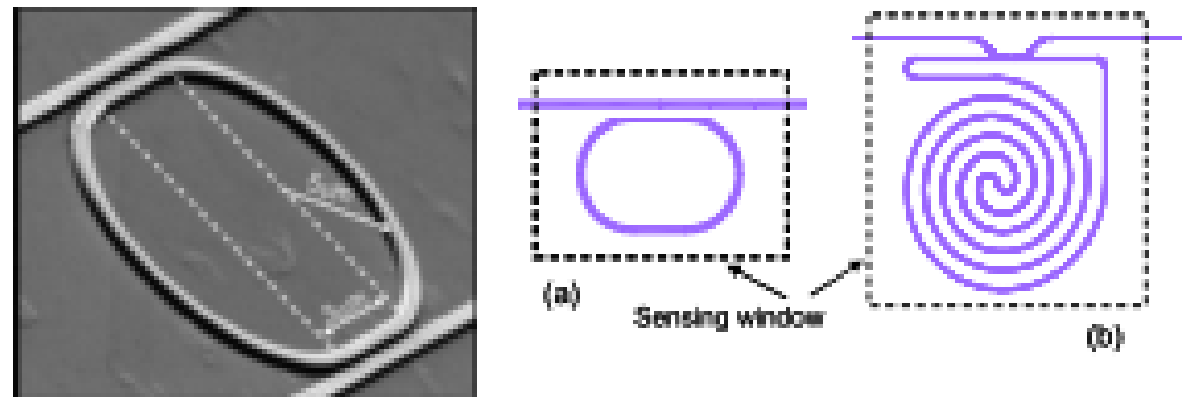

Figure 2: Right, SOI MRR optical biosensor demonstrated by Vos et al. in [43] Left, (a) regular MRR based optical sensors, (b) Folded cavity MRR optical cavity senor as demonstrated by D. Xu et al. [31]

The previously demonstrated devices used directional couplers. While directional couplers offer the ability to generate high Q-factor they are highly susceptible to fabrication errors. The MMI couplers on the other hand have a wide fabrication tolerance which is ideal for the large scale production. D. Xu et al. demonstrated a MMI coupler based micro ring resonators with a Q-factor from 6,000-7,000, bandwidths as low as $0.22 \mathrm{~nm}$, and extinction ratios of $12 \mathrm{~dB}$ to $28 \mathrm{~dB}$. The designs used by Xu et al. used rings with radii of $5 \mu \mathrm{m}$ and $50 \mu \mathrm{m}$ and MMI couplers with sizes of $3 \mu \mathrm{m} \times 9 \mu \mathrm{m}$ and $4.5 \mu \mathrm{m} \times$ $20 \mu \mathrm{m}$. The larger MMI devices offered higher Q-factors and extinction ratios than the smaller. Xu et al. indicate as well that with an uneven splitting ratio a higher $\mathrm{Q}$ factor can be achieved and further optimization to the device dimension would improve the performance of the resonators.

The detection limit of the MRR sensors is limited by the Q-factor of the device, which decreases the effect of the noise when determining the resonant wavelength. The ability to increase the Q-factor is limited by the high susceptibility of the MRR sensors to fabrication errors. Thus another method to increase the detection limit of the device is required. This can be done by changing the waveguide geometry. This was demonstrated 
by C. A. Barrios et al. [44]. C. A. Barrios et al. used a slot waveguide geometry for a ring resonator. The slot waveguide was first theorized by V. R. Almeida et al. [45] and consists of two rectangular waveguides in close proximity to each other with subwavelength gap between them. The light is then confined in the gap which allows for a large interaction between the sensor and the sensing medium. C. A. Barrios et al. produced a slot waveguide MRR on the $\mathrm{Si}_{3} \mathrm{~N}_{4}-\mathrm{SiO}_{2}$ platform. They were able to achieve a sensitivity of $212 \mathrm{~nm} / \mathrm{RIU}$ with a minimum sensitivity of $2 \times 10^{-4}$ RIU. This result was two times higher than conventional wire waveguide MRR. C. A. Barrios et al. also determined via simulations that the slot was only partially filled and with a better flowinjection system the better results could be achieved. This result is a positive indication of the potential for slot-waveguide based MRR as biochemical sensors.

T. Claes et al. demonstrated the first slot-waveguide based MRR on the SOI platform [28]. T. Claes et al. present a SOI slot-waveguide based MRR with a radius of $5 \mu \mathrm{m}$ and using DCs. The waveguides consist of two sections of silicon that are $268 \mathrm{~nm} \mathrm{x}$ $220 \mathrm{~nm}$ with a slot width of $104 \mathrm{~nm}$. They were able to achieve a bulk index sensitivity of $298 \mathrm{~nm} / \mathrm{RIU}$ with a detection limit of $4.2 \times 10^{-5}$ RIU. Like C. A. Barrios before them, they determined via simulation that the gap in the waveguide was partially filled, with simulations showing a potential sensitivity of $348 \mathrm{~nm} / \mathrm{RIU}$ with the gap fully filled. T. Claes et al. also tested the device as a protein sensor. They were able to detect avidin $100 \mu \mathrm{g} / \mathrm{ml}$ in a saline buffered solution, with a resonant shift of $2.2 \mathrm{~nm}$ which was 3.5 times that of the normal waveguide based MRR sensors demonstrated by K. De Vos et al. They also proposed that with lower waveguide losses and bending losses (by increasing 
the radius of the rings) in the device, performance would be increased greatly due to the improvement to the Q-factor.

The increase in radius of the rings will increase the Q-factor but will decrease the free spectral range of the device, thus limiting the range of measurements possible. D. Dai and S. He [32] demonstrate via simulations a ring assisted Mach-Zehnder interferometer for sensing. This was a design previous used as a thermo-optic switch and reconfigurable filter in [46], [47] respectively. With this design it is possible to control the length difference between two arms of the MZI in such a way to have one resonant wavelength with a very high extinction ratio for a large wavelength span or quasi-FSR. Using TMM simulations D. Dai and S. He find a sensitivity of $372.55 \mathrm{~nm} / \mathrm{RIU}$ with a large measurement range of up to 0.48 RIU. This design was tested experimentally by J. Wang and D. Dai [33] in which the they use wire-waveguide based MRR with a ring radius of $2 \mu \mathrm{m}$ with DC. Their device showed a high extinction ratio of $16-36 \mathrm{~dB}$ for the major resonant peaks with a quasi-FSR of over $120 \mathrm{~nm}$. The device achieved a sensitivity of $111 \mathrm{~nm} / \mathrm{RIU}$ over a refractive index range of 1.0 to $1.538 \mathrm{RIU}$.
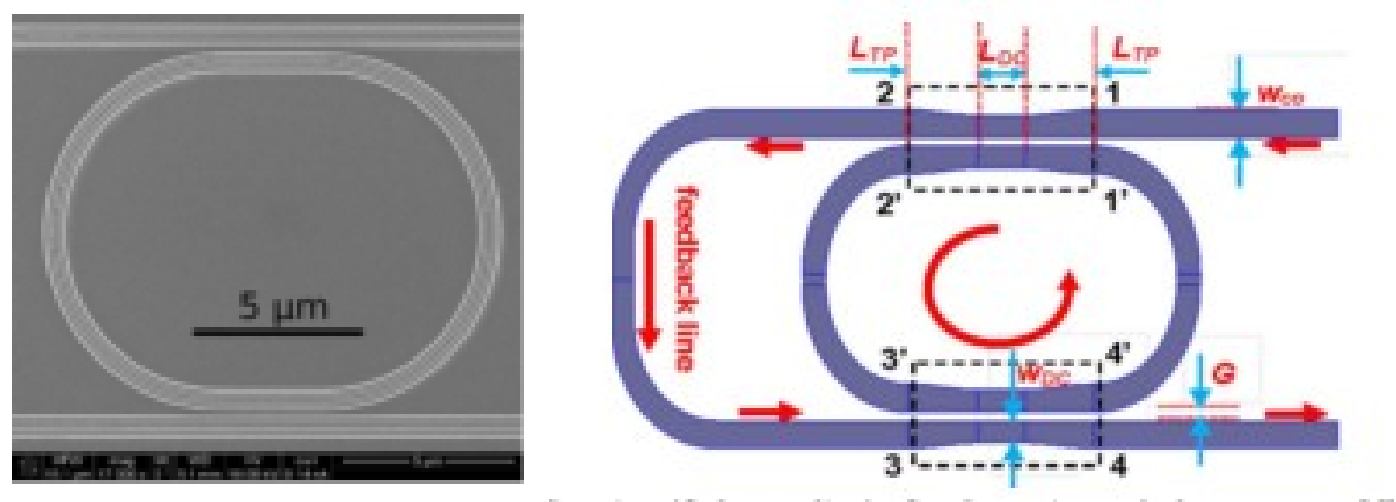

Figure 3: Left, slot based MRR chemical sensors demonstrated by T. Claes et al.[28] Right, RAMZI based chemical sensor demonstrated by D. Dai in [33] 
The literature shows that the MRR design has demonstrated excellent performance a biochemical sensor with a sensitivity $70 \mathrm{~nm} / \mathrm{RIU}$ with wire waveguide and $298 \mathrm{~nm} / \mathrm{RIU}$ when the slot waveguide was used. These devices have only used DCs which do offer superior Q-values while being susceptible to fabrication variations. On the other hand, the MMI coupler offers increased fabrication tolerance and with proper design can produce high Q-value performance. Compared to the simple MRR configuration, the more recently presented RAMZI design allows for increased quasi-FSR allowing for a large measurement range while keeping a relatively high sensitivity of $111 \mathrm{~nm} / \mathrm{RIU}$. 


\section{Chapter: Theory and Concepts}

In this chapter we discuss the operating theory of the basic photonic devices required to implement the MRR and RAMZI devices, as well the theory of the MRR and RAMZI devices. The theory of optical chemical sensors is discussed at the end of the chapter.

\subsection{Waveguides}

The waveguide is a fundamental optical device which allows light to be guided along a selected path. The most basic design for a waveguide is that of the slab waveguide. This consists of three dielectric layers stacked on top of each other. This design is a good demonstration of the theory behind guiding of waves. However it is not practical as it only confines the wave in one dimension. For practical use in photonic integrated circuits the wave must be confined in two dimensions.

For silicon photonic circuits the two main designs for waveguides are that of the ridge waveguide and wire waveguide. In recent years the slot waveguide has been designed and demonstrated. This this design allows for greater confinement of light than the conventional waveguide [45].

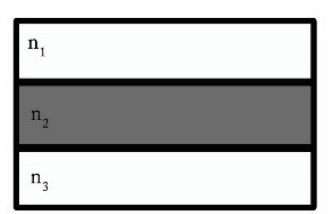

Slab Waveguide

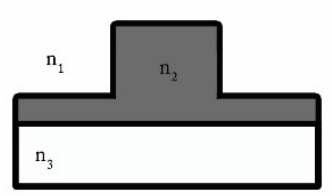

Rib Waveguide

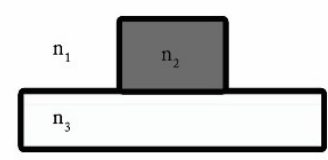

Wire Waveguide

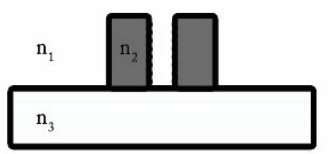

Slot Waveguide

Figure 4: Schematic drawing for the four common waveguide designs 


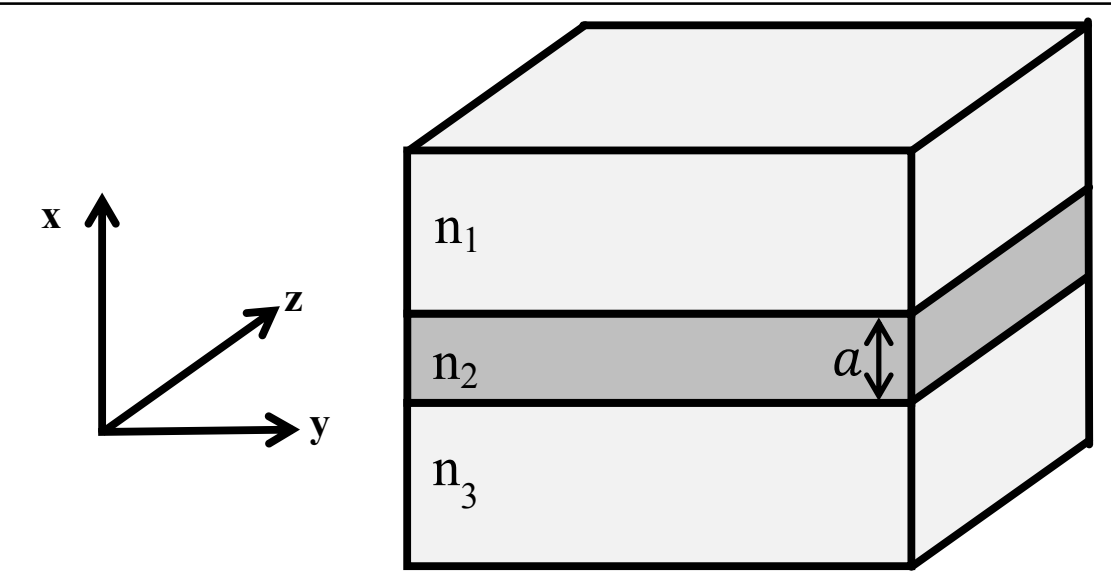

Figure 5: Schematic of a Slab waveguide

The slab illustrated in Figure 5 consists of the three dielectric layers with the top layer having an index of refraction of $n_{1}$, the middle layer having an index of refraction $n_{2}$, and the bottom layer, $n_{3}$. The Maxwell's equation for the magnetic field vector $(\boldsymbol{H})$ and electric field vector $(\boldsymbol{E})$ can be written as,

$$
\begin{aligned}
\boldsymbol{\nabla} \times \boldsymbol{H}=i \omega \varepsilon_{0} n^{2} \boldsymbol{E} \\
\boldsymbol{\nabla} \times \boldsymbol{E}=-i \omega \mu \boldsymbol{H}
\end{aligned}
$$

Where $n$ is the effective index profile described previously, $\omega$ is the angular frequency, $\varepsilon_{0}$ is the permittivity of free space, and $\mu$ the permeability of the material.

Considering a monochromatic wave propagating in the $n_{2}$ layer along the z-axis, the mode of propagation in the waveguide can be separated into two categories; the transverse electric mode (TE), and the transverse magnetic (TM) mode. In the TE mode, the electric field vector is transverse to the plane formed by the direction of propagation and axis along which the dielectric varies. Likewise in the TM mode the magnetic field vector is transverse to the plane. First looking at the TE mode we can find the general solution for the slab waveguide to be [48], 


$$
\boldsymbol{E}_{\boldsymbol{y}}(x)=\left\{\begin{array}{cc}
A e^{-q x} & x \leq 0 \\
A\left(\cos (h x)+\frac{q}{h} \sin (h x)\right) & -a \leq x \leq 0 \\
A\left(\cos (h a)+\frac{q}{h} \sin (h a)\right) e^{p(x+a)} & x \leq-a
\end{array}\right.
$$

Where $\mathrm{A}$ is the amplitude of the wave, and $q, p$, and $h$ are defined by,

$$
\begin{aligned}
& q=\sqrt{\beta^{2}-n_{1}^{2} k^{2}} \\
& p=\sqrt{\beta^{2}-n_{3}^{2} k^{2}} \\
& h=\sqrt{n_{2}^{2} k^{2}-\beta^{2}}
\end{aligned}
$$

Where $\beta$ is the propagating wavenumber in the medium, and $\mathrm{k}$ wavenumber in a vacuum. At dielectric interfaces boundary conditions must be observed; the tangential electric and magnetic field components across the dielectric interface are continuous. By applying the continuity condition to the $\boldsymbol{E}_{\boldsymbol{y}}$ and $\boldsymbol{H}_{\boldsymbol{z}}$ components we can determine the mode condition for the TE mode to propagate through the waveguide to be defined as,

$$
\tan (h a)=\frac{p+q}{h\left(1-p q / h^{2}\right)}
$$

Using the same method we can find the TM mode condition to be,

$$
\tan (h a)=\frac{h\left(\frac{n_{2}^{2}}{n_{3}^{2}} p+\frac{n_{2}^{2}}{n_{1}^{2}} q\right)}{\left(h^{2}-\frac{n_{2}^{2}}{n_{3}^{2}} \frac{n_{2}^{2}}{n_{1}^{2}} p q\right)}
$$

Both the TE and TM mode conditions can be satisfied for multiple values of $\beta$. These solutions are known as modes. Each mode has a different propagation constant. Most photonic integrated circuits are only designed to allow for the first order mode to 
propagate. At cutoff $p=0$ and $\beta=n_{3} \omega / c$ we can then find the ratio of slab thickness to wavelength, $\left(\frac{a}{\lambda}\right)$ for the $m$ th order confined mode to be,

$$
\begin{aligned}
& \left(\frac{a}{\lambda}\right)_{T E}=\frac{1}{2 \pi \sqrt{n_{2}^{2}-n_{3}^{2}}}\left[m \pi+\tan ^{-1}\left(\frac{n_{3}^{2}-n_{1}^{2}}{n_{2}^{2}-n_{3}^{2}}\right)^{\frac{1}{2}}\right] \\
& \left(\frac{a}{\lambda}\right)_{T M}=\frac{1}{2 \pi \sqrt{n_{2}^{2}-n_{3}^{2}}}\left[m \pi+\tan ^{-1} \frac{n_{2}^{2}}{n_{1}^{2}}\left(\frac{n_{3}^{2}-n_{1}^{2}}{n_{2}^{2}-n_{3}^{2}}\right)^{\frac{1}{2}}\right]
\end{aligned}
$$

This is the simplest case for a waveguide design can is easily solved analytically the $2 \mathrm{D}$ waveguide geometries however do not have simple analytical solutions and require simulations (FDTD, FEM, etc.) to determine the propagating modes within the waveguides.

The original solution only considers the index of refraction being a real number however in reality it is a complex value. Considering the general solution for a wave,

$$
E(r, t)=E_{0} e^{j(k r-\omega t)}
$$

Where $r$ is the position on the wave, $t$ is time, $k=2 \pi / \lambda=n k_{0}$ is the wavenumber in a vacuum, and $\omega$ is the angular frequency. Now if we consider the index of refraction to be complex $n=n_{r}-i n_{i}$, where $n_{r}$ is the real portion of the index and $n_{i}$ is the imaginary portion of the index of refraction, and assuming the wave to be propagating along the $\mathrm{z}$ axis we find,

$$
E=E_{0} e^{-k_{0} n_{i} z} e^{j\left(k_{0} n_{r} z-\omega t\right)}
$$

From this we can see that the equation is same as the ideal case but with the $e^{-k_{o} n_{i}}$ term. This term is referred to as the loss term, with the intensity loss coefficient begin defined as $\alpha=2 k_{0} n_{r}$. The loss coefficient is affected by a number of reasons; scattering, absorption and radiation. Scattering is the loss due to light being scattered from 
imperfections in the optical medium and roughness at the interfaces of the core and cladding of the waveguide. Absorption in silicon waveguides is mainly due to interband absorption and free carrier absorption. Interband absorption occurs when the photons have sufficient energy to excite electrons from the valance to the conduction band. This is easily avoided by operating with a wavelength of light about $1.1 \mu \mathrm{m}$. Free carrier absorption is due to the change in index of refraction due to the concentration of free

carriers in the medium. The last cause of loss is radiation which is caused by light leaking into the cladding from the core. This can be avoiding be the proper design of the waveguides [49].

\subsection{Optical Couplers}

Optical couplers are a fundamental building block of photonic integrated circuits. They allow light to be split or transferred from one waveguide to another. The two most commonly used couplers are the directional coupler (DC) and multimode interference coupler (MMI). The DC offers better performance but with lower fabrication tolerances whereas the MMI does not perform as well as the DC but is more tolerant to fabrication errors.

\subsubsection{Directional Coupler}

A DC consists of two single mode waveguides in close proximity to each other. The optical power is exchanged between waveguides due to modal interaction of the evanescent fields of the waveguide. The basic schematic for the coupler can be seen in Figure 6. 


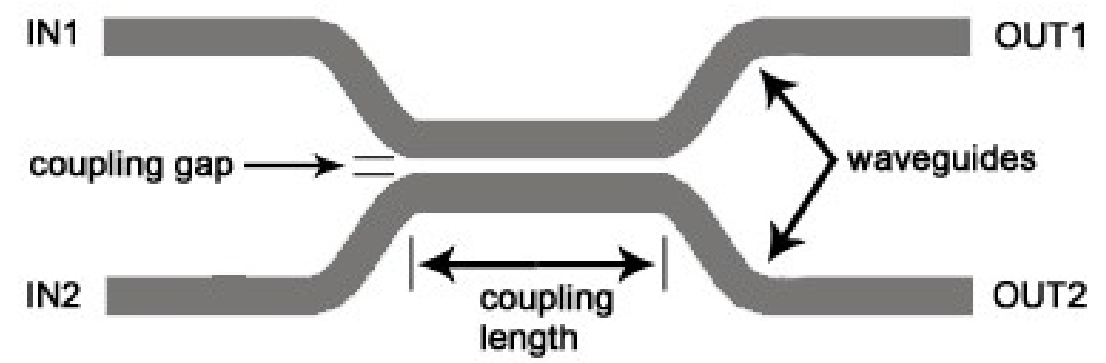

Figure 6: Schematic of the Directional Coupler

The coupling between the waveguides can described by a approximate solution of the wave equation using perturbation theory of coupled modes, [50]-[52]

$$
E(x, y, z, t)=a(z) \varepsilon_{a}(x, y) e^{i\left(\omega t-\beta_{a} z\right)}+b(z) \varepsilon_{b}(x, y) e^{i\left(\omega t-\beta_{b} z\right)}
$$

Where $\varepsilon_{a}(x, y)$ and $\varepsilon_{b}(x, y)$ are the transverse electric field profiles for the two guided modes in the waveguides, and $\beta_{a}$ and $\beta_{b}$ are the propagation constants for the two waveguides, $a(z)$ and $b(z)$ are the amplitudes of the evanescent coupling between the two waveguides along the length, $\mathrm{z}$ of the $\mathrm{DC}$, and $\omega$ is the angular frequency. From perturbation theory the amplitudes are govern by,

$$
\begin{gathered}
\frac{d R(z)}{d z}=i \delta R(z)-i \kappa S(z) \\
\frac{d S(z)}{d z}=-i \delta S(z)-i \kappa R(z)
\end{gathered}
$$

Where $R(z)=a(z) e^{-i \delta z}, S(z)=b(z) e^{i \delta z}, \delta=\left(\beta_{a}-\beta_{b}\right) / 2$, and $\kappa$ is the coupling coefficient normally defined by the overlap of the evanescent field profiles,

$$
\kappa=\frac{\omega}{4} \varepsilon_{0} \iint_{-\infty}^{\infty} \varepsilon_{a}(x, y) \Delta n^{2}(x, y) \varepsilon_{b}(x, y) d x d y
$$

Where $\Delta n^{2}(x, y)$ is the change in index of refraction due to the perturbation. Considering the case for a symmetric DC $\varepsilon_{a}(x, y)=\varepsilon_{b}(x, y)$, and $\beta_{a}=\beta_{b}$ we can reduce Eq. ( 11$)$ and solved for inputs at $\mathrm{z}=0, R_{0}$ and $S_{0}$, 


$$
\left[\begin{array}{l}
R(z) \\
S(z)
\end{array}\right]=\left(\begin{array}{cc}
\mathrm{t} & i k \\
i k & \mathrm{t}
\end{array}\right)\left(\begin{array}{l}
R_{0} \\
S_{0}
\end{array}\right)
$$

Where $t=\cos (\kappa z)$ and $k=-\sin (\kappa z)$ which are the self- and cross-coupling coefficients. From this we can see that the transfer matrix is unitary indicating the symmetric DC operates independently of the direction of incoming light, as well the two outputs have a relative shift of $\pi / 2$, and for the first order mode the coupling length being $L_{c}=\pi / 2 \kappa$

This solution however does not include the losses of the waveguides or the interaction of the fan-in and fan-out waveguides. The losses affect the performance of the device. The bending of the fan-in and fan-out waveguide causes the coupling coefficient ,which was assumed to be constant, to vary along $z$. In the coupled mode theory this can be compensating for by introducing an additional effective length, determined using empirical data. For numerical method techniques this will be accounted for automatically.

\subsubsection{Multimode Interference Coupler}

The MMI uses the principal of self-imaging within a multimode waveguide to couple light [53], [54]. The typical design for a MMI coupler supports more than 3 modes and is referred to as an $\mathrm{N} x \mathrm{M}$ MMI coupler with $\mathrm{N}$ being the number of input waveguides and $\mathrm{M}$ being the number of output waveguides.

Since the waveguide's width is much larger than the thickness we can reduce the problem to $2 \mathrm{D}$ and using modal propagation method we can find the spacing of the fundamental modes the higher order modes to be approximated to be,

$$
\beta_{0}-\beta_{m}=\frac{m(m+2) \pi}{3 L_{\pi}}
$$

Where $m$ is the number of the higher order mode, $L_{\pi}$ is the beat length of the interference pattern which can be found to be, 


$$
L_{\pi}=\frac{4 n_{r} W_{e f f}^{2}}{3 \lambda}
$$

Where $n_{r}$ the effective is refractive index of the core, and $W_{\text {eff }}$ is the effective width of the waveguide which can be defined by,

$$
W_{e f f}=W+\frac{\lambda}{\pi}\left(\frac{n_{c}}{n_{r}}\right)^{2 \sigma}\left(n_{r}^{2}-n_{c}^{2}\right)^{-\frac{1}{2}}
$$

With $\sigma=0$ for TE and $\sigma=1$ for TM polarizations, $\lambda$ is the wavelength, $W$ is the width of the MMI coupler and $n_{c}$ is the effective index of the cladding of the device.

Within photonics circuits the most common usage for the coupler is as a $3 \mathrm{~dB}$ splitter in either the $1 \times 2$ or $2 \times 2$ configuration. For the $1 \times 2 \mathrm{MMI}$ with the input waveguide centered with respect to the multimode waveguide and due to the symmetry of the waveguide only the $m=0,2,4, \ldots$ modes are excited with $\mathrm{M}$-fold image found for a length of $3 L_{\pi} / 4 M$. For our purposes the $2 \times 2 \mathrm{MMI}$ is of greater importance. In this configuration the $\mathrm{M}$-fold images are found at $p\left(3 L_{\pi}\right) / M$ where $p \geq 0$. For even values of $p$ we have self-imaging and for odd values we see mirrored self-imaging, and $p$ and $M$ have no common divisor. From this we can see the $3 \mathrm{~dB}$ coupling, the 2 -fold image will occur for a length of $3 L_{\pi} / 2$.
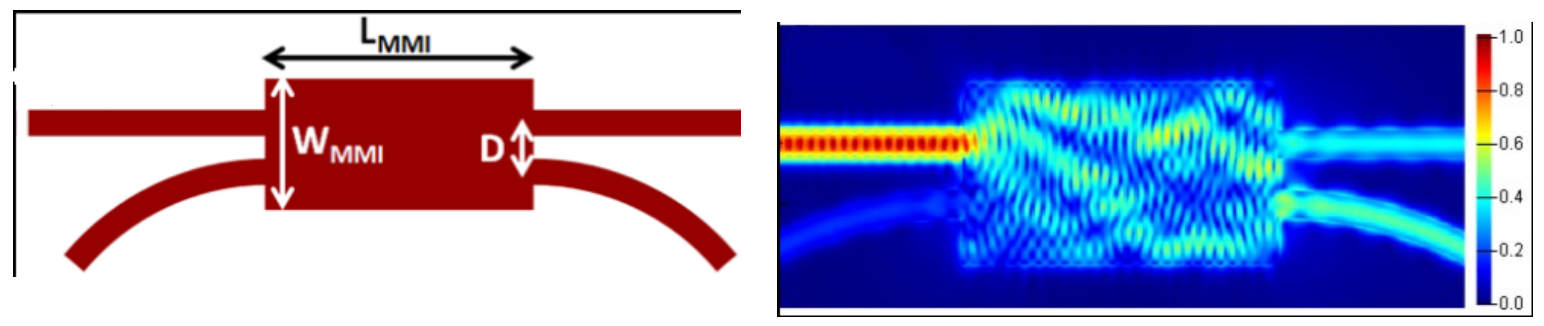

Figure 7: Left, Schematic of the MMI coupler. Right. Simulation of the MMI coupler.

\subsection{Ring Resonators}

A ring resonator consists of a looped waveguide and a coupler to allow light into the loop. When the phase shift through the ring is equal to an integer value $2 \pi$ the waves 
cause constructive interference and the cavity is at resonance. The two designs of rings of interest are the single coupler design and the double coupler design, both can be seen in Figure 8 .
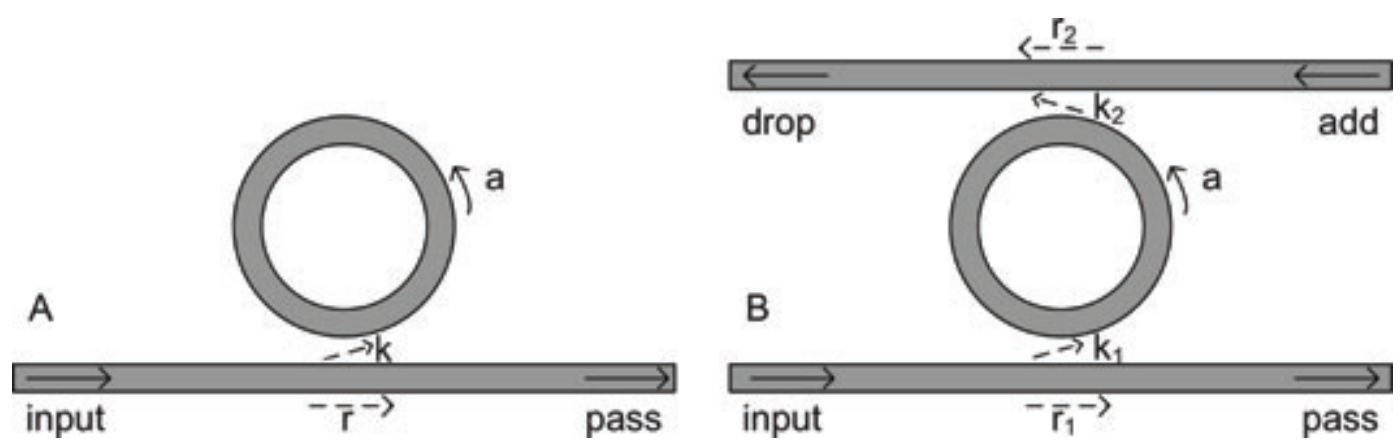

Figure 8: A) single coupler design, B) double coupler design [55]

Using the matrix transfer method for the single coupler design we can find the output, from Eq. ( 13 ) we know the general form of the coupler output.

$$
\left[\begin{array}{l}
E_{4}(\omega) \\
E_{2}(\omega)
\end{array}\right]=\left[\begin{array}{cc}
t & i k \\
i k & t
\end{array}\right]\left[\begin{array}{l}
E_{3}(\omega) \\
E_{1}(\omega)
\end{array}\right]
$$

Where $E_{1}$ is the incident, $E_{2}$ is the transmitted, $E_{3}$ and $E_{4}$ are the circulating fields and $t$ and $k$ are the self- and cross-coupling coefficients respectively. And we can relate the circulating fields by the following expression,

$$
E_{3}=e^{-\frac{a}{2} 2 \pi R} e^{i k 2 \pi R} E_{4}=a e^{-i \phi} E_{4}
$$

Where $R$ is the radius of the ring, and $a$ represnts the amplitude transmission for a single pass around the ring and $\phi$ represents the phase shift of a single pass around the ring. We can then take the ratio of transmitted and incident fields in the bus waveguide to be [55],

$$
\frac{E_{2}}{E_{1}}=e^{i(\pi+\phi)} \frac{\left(a-t e^{-i \phi}\right)}{1-t a e^{i \phi}}
$$

Taking the absolute square of this we can find the transmission trough the device to be,

$$
T_{n}=\frac{a-2 r a \cos \phi+t^{2}}{1-2 t a \cos \phi+(t a)^{2}}
$$


From this we see that the resonance occurs when the phase is a multiple of $2 \pi$, or equivalently the length of the ring is equal to an integer value of the optical length of the ring,

$$
\lambda_{r}=\frac{n_{e f f} L}{m}, \quad m=1,2,3, \ldots
$$

Where $n_{\text {eff }}$ is the effective index of the waveguide, $\mathrm{L}$ is the optical path length of the micro ring resonator, and $m$ is the order of excitement of the optical cavity.

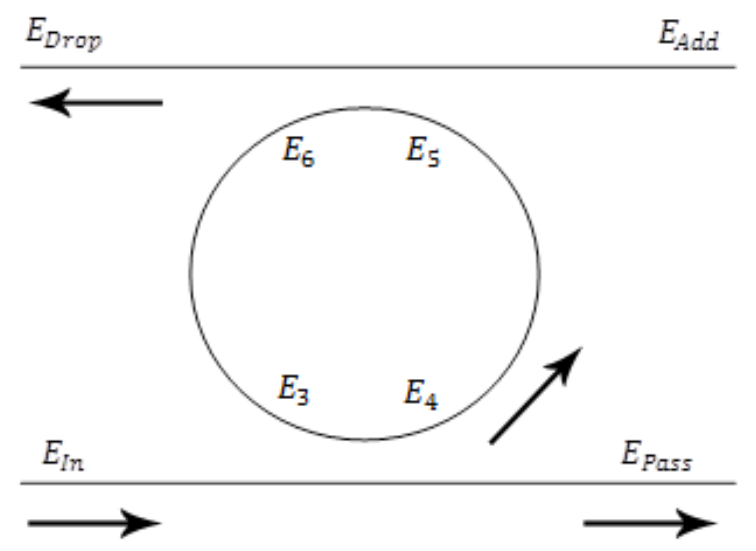

Figure 9: Schematic of the double coupler MRR

Now, looking at the double coupler design we can write the two matrices for each coupler to be,

$$
\begin{aligned}
{\left[\begin{array}{c}
E_{4}(\omega) \\
E_{\text {Pass }}(\omega)
\end{array}\right] } & =\left[\begin{array}{cc}
t_{1} & i k_{1} \\
i k_{1} & t_{1}
\end{array}\right]\left[\begin{array}{c}
E_{3}(\omega) \\
E_{\text {In }}(\omega)
\end{array}\right] \\
{\left[\begin{array}{c}
E_{6}(\omega) \\
E_{\text {Drop }}(\omega)
\end{array}\right] } & =\left[\begin{array}{cc}
t_{2} & i k_{2} \\
i k_{2} & t_{2}
\end{array}\right]\left[\begin{array}{c}
E_{5}(\omega) \\
E_{\text {Add }}(\omega)
\end{array}\right]
\end{aligned}
$$

Where $t_{1}$ and $t_{2}$ are the through coupling coefficients of the two couplers, $k_{1}$ and $k_{2}$ are the cross-coupling coefficients of of the two couplers, and the electric fields are defined in Figure 9 we can relate $E_{4}$ to $E_{5}$ and $E_{3}$ to $E_{6}$ with the same relationship as in Eq. ( 18 ). However the equation should be altered so that the phase shift is only $\frac{1}{2} \phi$ and redefine 
$a$ to be the loss through half the ring since they are only half way around the loop. The transmission for the Pass and Drop port can then be found to be,

$$
\begin{aligned}
& T_{\text {pass }}=\frac{k_{2}^{2} a^{2}-2 k_{1} k_{2} \cos \phi+k_{1}^{2}}{1-2 k_{1} k_{2} a \cos \phi+\left(k_{1} k_{2} a\right)^{2}} \\
& T_{\text {drop }}=\frac{\left(1-k_{1}^{2}\right)\left(1-k_{2}^{2}\right) a}{1-2 k_{1} k_{2} a \cos \phi+\left(k_{1} k_{2} a\right)^{2}}
\end{aligned}
$$

From this we can see again that the resonance will occur when the phase shift around the waveguide is equal to an integer multiple of $2 \pi$.

The performance of the MRR is typically defined by five values; Full Width Half Max (FWHM), Free spectral range (FSR), extinction ratio (ER), Finesse, and Quality factor (Q-factor). FWHM is the width of the resonant peak at a half height of the peak, for a single coupler MRR and double coupler MRR designs can be derived from Eq. (21),(24), and (25) and found to be,

$$
\begin{gathered}
F W H M=\frac{(1-k a) \lambda_{r}^{2}}{\pi n_{g} L \sqrt{k a}} \\
F W H M=\frac{\left(1-k_{1} k_{2} a\right) \lambda_{r}^{2}}{\pi n_{g} L \sqrt{k_{1} k_{2} a}}
\end{gathered}
$$

Here $k, k_{1}$ and $k_{2}$ are the cross-coupling coefficients for the single and double coupler ring resonators, a is the loss through the ring, $\mathrm{L}$ is the optical path length and $n_{g}$ is the group index which is takes into account the dispersion of the waveguide, and $\mathrm{L}$ is the length of the waveguide. Free spectral range is the distance between the two adjacent resonant peaks this can be found by a first order approximation of the dispersion,

$$
F S R=\frac{\lambda_{r}^{2}}{n_{g} L}
$$


Where $\lambda_{r}$ is the resonant wavelength of the first peak, $n_{g}$ is the group index of the waveguide, and $\mathrm{L}$ is the optical path length.

The extinction ratio of the MRR is the on/off transmission peak ratio of the device. For the single coupler waveguide this would be $\frac{T_{t}}{R_{\min }}$. For the double coupler there are two ER values, one for the pass port and one for the drop port. These are defined respectively as $\frac{T_{\max }}{T_{d}}$ and $\frac{T_{\max }}{R_{\min }}$.

The final two values which are used to characterize the MRR are Finesse and Qfactor. Although these values are different, they are used for the same purpose: to define the sharpness of the resonant peaks. Finesse is the sharpness of the resonances relative to the FSR and is defined as,

$$
\text { Finesse }=\frac{F S R}{F W H M}
$$

Whereas the Q-factor is a measure of sharpness of the resonant peaks relative to the resonant wavelength and is defined as,

$$
Q-\text { factor }=\frac{\lambda_{r}}{F W H M}
$$

From this it can be seen that the length of the resonator has as an inverse relation to the both FWHM and FSR and since FWHM is inversely portioned to the Finesse and Qfactor it is impossible to have MRR with both a large Q value and Large FSR.

\subsection{Mach-Zehnder Interferometer}

The Mach-Zehnder interferometer is another fundamental building block of photonic integrated circuits. It has been used as the basis for modulators, switches and filters. The basic design consists of an input waveguide which splits the light into two arms of light and an output waveguide which recombines the arms. This will cause an 
interference pattern to form based on differences in the paths the light has to take. The schematic of this can be seen in Figure 10.

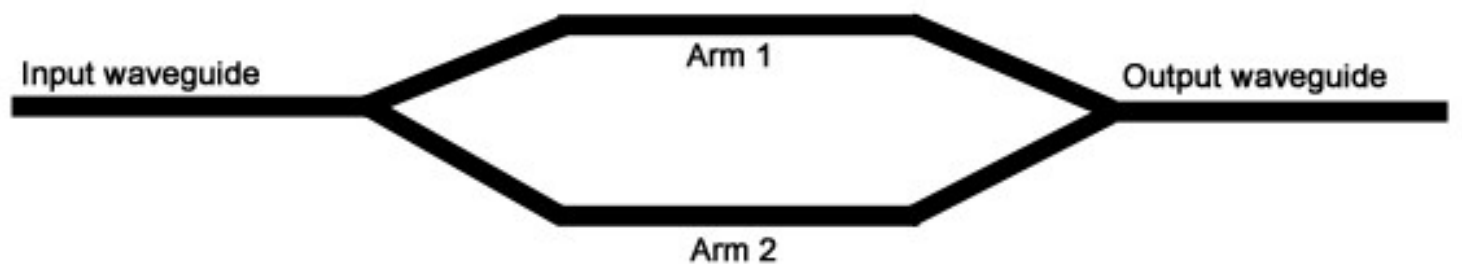

Figure 10: Schematic of the MZI device[49]

Consider an input wave with a TE polarization which is split at the first junction. Assuming the input is split evenly, we can define the electric fields $E_{1}$ and $E_{2}$ in the arms as,

$$
\begin{aligned}
& E_{1}(z, t)=E_{0} \sin \left(\omega t-\beta_{1} z\right) \\
& E_{2}(z, t)=E_{0} \sin \left(\omega t-\beta_{2} z\right)
\end{aligned}
$$

Where $\mathrm{E}_{0}$ is the amplitude of the two waves, $\omega$ is the angular frequency of light and $\beta_{1}$ and $\beta_{2}$ are propagation constants for the two waveforms. As the waves pass through the different arms of the device, the phase is changed. This causes interference when the waves are recombined. The intensity of the light at the output will be,

$$
S_{T}=\left[\left(E_{1}+E_{2}\right) \times\left(H_{1}+H_{2}\right)\right]=S_{0}\left(E_{1}+E_{2}\right)^{2}
$$

Where $H_{1}$ and $H_{2}$ are the magnetic field for the two arms for the MZI, and $S_{0}$ is the amplitude of output intensity. And if the optical path length of the arms are $L_{1}$ and $L_{2}$ we can evaluate $\left(E_{1}+E_{2}\right)^{2}$ to be,

$$
\begin{aligned}
S_{T}=S_{0}\left(\mathrm{E}_{0}^{2} \sin ^{2}\left(\omega t-\beta_{1} L_{1}\right)+\mathrm{E}_{0}^{2} \sin ^{2}\left(\omega t-\beta_{2} L_{2}\right)\right. \\
\left.+2 \mathrm{E}_{0}^{2} \sin \left(\omega t-\beta_{1} L_{1}\right) \sin \left(\omega t-\beta_{2} L_{2}\right)\right)
\end{aligned}
$$

Using trigonometric identities and reducing the equation to the time averaged result yields the following equation, 


$$
S_{T}=S_{0} E_{0}^{2}\left(1+\cos \left(\beta_{2} L_{2}-\beta_{1} L_{1}\right)\right)
$$

From this we see that if the arms have the same waveguide design the interference is dependent only on the difference in length of the two arms.

\subsection{Ring Assisted Mach-Zehnder Interferometer}

The RAMZI device is a Mach-Zehnder Interferometer in which one of the arms is a MRR. The schematic of this can be seen below in Figure 11.

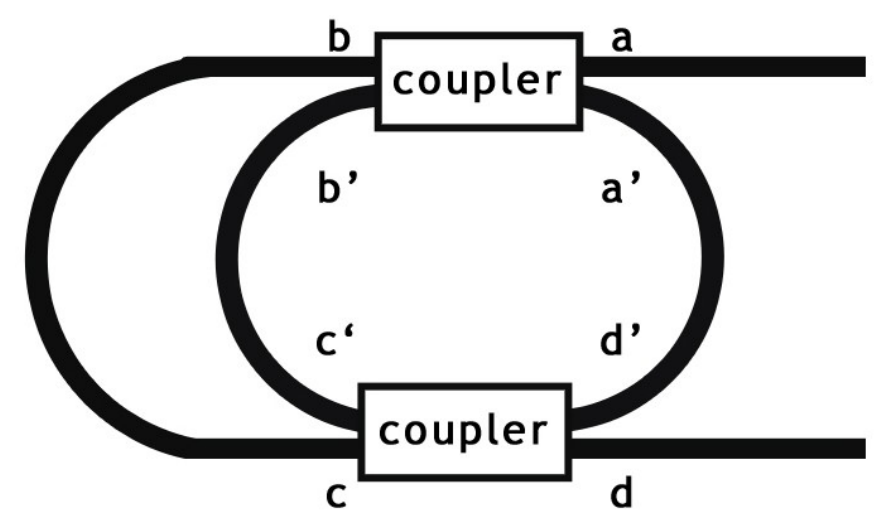

Figure 11: Schematic of ring-assisted MZI (RAMZI) racetrack design. The couplers can be directional couplers (DCs) or multimode interference (MMI) couplers.।

Using the matrix transfer method, the electric field distribution of the output of the RAMZI device can be found as,

$$
\left[\begin{array}{c}
E_{d} \\
E_{d^{\prime}}
\end{array}\right]=\left[\begin{array}{cc}
k_{c d} & k_{c^{\prime} d} \\
k_{c d^{\prime}} & k_{c^{\prime} d^{\prime}}
\end{array}\right]\left[\begin{array}{cc}
k_{b c} & 0 \\
0 & k_{b^{\prime} c^{\prime}}
\end{array}\right]\left[\begin{array}{cc}
k_{a b} & k_{a^{\prime} b} \\
k_{a b^{\prime}} & k_{a^{\prime} b^{\prime}}
\end{array}\right]\left[\begin{array}{c}
E_{a} \\
E_{a^{\prime}}
\end{array}\right]
$$

Where $E_{x}$ is the electric field distribution at the respective port of the device, and $k_{x y}$ is the transfer coefficient that relates the electric fields at the respective ports. If we assume the couplers are identical to each other, then we can define the self-coupling coefficient to be $t$ and cross-coupling coefficient to be $\kappa$, and the optical attenuation of the coupler to be $\alpha_{a b}$ and the equation can be reduced to, 


$$
\left[\begin{array}{c}
E_{d} \\
E_{d^{\prime}}
\end{array}\right]=\alpha_{a b}^{2}\left[\begin{array}{cc}
t & \kappa \\
-\kappa^{*} & t^{*}
\end{array}\right]\left[\begin{array}{cc}
k_{b c} & 0 \\
0 & k_{b^{\prime} c^{\prime}}
\end{array}\right]\left[\begin{array}{cc}
t & \kappa \\
-\kappa^{*} & t^{*}
\end{array}\right]\left[\begin{array}{c}
E_{a} \\
E_{a^{\prime}}
\end{array}\right]
$$

The transfer coefficients of $k_{b c}$ and $k_{b^{\prime} c^{\prime}}$ can be defined as by the propagation loss through the waveguides. Thus, $k_{b c}=\alpha_{b c} e^{\left(-i \beta L_{b c}\right)}$ and $k_{b^{\prime} c^{\prime}}=\alpha_{b^{\prime} c^{\prime}} e^{\left(-i \beta L_{b^{\prime} c^{\prime}}\right)}$, where $L_{b c}$ and $L_{b^{\prime} c^{\prime}}$ are the lengths of the $b c$ and $b^{\prime} c^{\prime}$ arms of the RAMZI device, and $\beta$ the propagation constant. Using these identities, the equation can be solved and the field transmission can be found as follows,

$$
\begin{gathered}
\frac{E_{d^{\prime}}}{E_{a}}=k_{a d}+\frac{k_{a^{\prime} d} k_{d^{\prime} a^{\prime}} k_{a d^{\prime}}}{1-k_{d^{\prime} a^{\prime}} k_{a^{\prime} d}} \\
k_{a d}=\alpha_{a b}^{2}\left(t^{2} \alpha_{b c} e^{\left(-i \beta L_{b c}\right)}+|\kappa|^{2} \alpha_{b^{\prime} c^{\prime}} e^{\left(-i \beta L_{b^{\prime} c^{\prime}}\right)}\right) \\
k_{a d^{\prime}}=\alpha_{a b}^{2}\left(-t \kappa^{*} \alpha_{b c} e^{\left(-i \beta L_{b c}\right)}-\kappa^{*} t^{*} \alpha_{b^{\prime} c^{\prime}} e^{\left(-i \beta L_{b^{\prime} c^{\prime}}\right)}\right) \\
k_{a^{\prime} d}=\alpha_{a b}^{2}\left(\kappa t \alpha_{b c} e^{\left(-i \beta L_{b c}\right)}+t^{*} \kappa \alpha_{b^{\prime} c^{\prime}} e^{\left(-i \beta L_{b^{\prime} c^{\prime}}\right)}\right)
\end{gathered}
$$

From this we can examine the transmission coefficient from port a to d,

$$
k_{a d}=\alpha_{a b}^{2} e^{\left(-i \beta L_{b^{\prime} c^{\prime}}\right)}\left(t^{2} \alpha_{b c} e^{(-i \beta \Delta L)}+|\kappa|^{2} \alpha_{b^{\prime} c^{\prime}}\right)
$$

Where $\Delta L$ is the length difference between the two arms of the MZI, the length of $b c$ arm to length of the $b{ }^{\prime} c$ ' arm. This shows that the arm length difference directly affects the transmission coefficient and thus the Q-factor of the RAMZI device. Therefore if a high Q-factor is desired the length difference should be chosen to maximize transmission coefficient, $k_{a d}$. And if a high extinction ratio is desired the transmission coefficient, $k_{a d}$ should mach the total roundtrip loss of the MRR, that is, the critical coupling of the MRR. Using these principles the device can be designed so that there are major resonances with large Q-factors and high extinction ratio spanning a large wavelength span with smaller resonant peaks between them in the transmission spectrum [32], [33], [56]. The wavelengths of the major resonant peaks $\left(\lambda_{r}\right)$ can be defined as, 


$$
n_{e f f} \Delta L=\left(m+\frac{1}{2}\right) \lambda_{r}
$$

Where $n_{e f f}$ is the effective index of the arms of the RAMZI, and $m$ is the interference order of the peak. From this the quasi-FSR can be approximated to be,

$$
F S R_{\text {quasi }}=\frac{\lambda_{r}^{2}}{n_{g} \Delta L}
$$

Where $n_{g}$ is the group index of the waveguide.

\subsection{Optical Waveguide sensing}

Optical sensing effects in the waveguides can be separated into two categories; bulk refractive index changes and surface absorption changes, both of which result in an effective index change in the waveguide [18], [41], [42]. The change of effective index, $\delta n_{\text {eff }}$ of the device can be defined by variational theorem of waveguides as [52]

$$
\delta n_{e f f}(x, y)=c \int \Delta \varepsilon(x, y) E_{v}(x, y) \cdot E_{v}^{*}(x, y) d x d y
$$

Where $E_{v}(x, y)$ is the normalized electric field vector for the propagating mode of the waveguide, and $\Delta \varepsilon(x, y)$ is the change the local change in the dielectric constant. From this we can see the sensors response scales squared to the amplitude of the electric field at the perturbations, therefore only the evanescent field, which is a small fraction of the total electric field affects the sensing performance of optical waveguide sensors. The strength of the evanescent field can be increased by reducing the core thickness of the waveguide to decrease the mode confinement, thus increasing the interaction with cladding material and the performance of the sensor. Sensing can also be increased by using materials with high contrast in refractive index, and substituting the TM mode for the TE mode, since the dominant field components are polarized normal to the interface. 
From Eq. ( 21 ) we can see the change in effective index will shift the resonant wavelength of the MRR. The expression for sensitivity for a simple MRR can be written as [57]

$$
\Delta \lambda=\frac{L}{m} \Delta n_{e f f}=\frac{\lambda_{r}}{n_{e f f}} \Delta n_{e f f}
$$

Where $\Delta \lambda$ and $\Delta n_{\text {eff }}$ are the peak resonance shift and effective index shift caused by the cladding medium, respectively. This expression does take dispersion into account. To do this, we use the group index. With a first order approximation for the change in effective index caused by the changes cladding medium, the group index $n_{g}$ is:

$$
n_{g}=n_{e f f}-\lambda_{r} \frac{d n_{e f f}}{d \lambda}
$$

Hence the sensitivity for a simple MRR becomes

$$
\Delta \lambda=\frac{\lambda_{r} \Delta n_{e f f}}{n_{g}}
$$

Similarly, the expression for the sensitivity of a MZI device could be derived using eq. (38),

$$
\Delta \lambda=\frac{\Delta L}{p+1 / 2} \Delta n_{e f f}=\frac{\lambda_{r}}{n_{e f f}} \Delta n_{e f f}=\frac{\lambda_{r} \Delta n_{e f f}}{n_{g}}
$$

Here we see that Eq. (44) for MZI is identical to Eq. (43) for MRR. We can also see that the sensitivity is not dependent on the waveguide length. Hence, theoretically the RAMZI device should have the same sensitivity of a simple ring; however, the key advantage for a RAMZI device is the large quasi-FSR which allows for a large range of measurements for the device. 


\subsection{Conclusion}

In this chapter we reviewed the theoretical principals of the basic photonics devices, waveguides, DC and MMI couplers, and MZIs. We also examine the theoretical performance and spectral characteristics of the MRR and RAMZI devices, the RAMZI devices offers a similar Q values but allows for the increased FSR due to the fact the quasi-FSR is only based on the difference in the lengths of the arms and not in the size of

the micro ring in the device. Finally we reviewed the principles of optical sensing for photonic devices. 


\section{Chapter: Design and Fabrication}

\subsection{Device Design}

In this chapter we discuss the design of our optical chemical sensors, the fabrication technologies which were used to implement the devices, and the simulated performance of the coupler designs. Our designs were inspired by those proposed by D. Dai and S. He in [32] and demonstrated in [33] where wire waveguides were used to offer a decent sensitivity of $110 \mathrm{~nm} / \mathrm{RIU}$ with a measurement range of $1<n<1.538$. The designs proposed in this thesis are intended to demonstrate improved sensitivity of these devices through the use of a slot waveguide, and show the effects of different coupler designs on sensing performance. The designs for this project were first proposed by Yule Xiong in 2013 [56], [58].

Two waveguide designs were implemented for testing; a wire waveguide and a slot waveguide. The wire waveguides were used as a control to provide a basis for evaluating of the performance change due to the implementation of slot waveguides. The wire waveguides have height of $220 \mathrm{~nm}$ due to the etch depth constraints of the fabrication facility. For the waveguide to be single mode, our waveguides needed to be approximately $450 \mathrm{~nm}$ wide. The gap in the slot waveguides was chosen to be $180 \mathrm{~nm}$, which is the minimum feature size of the fabrication facility in the public foundry service. The $180 \mathrm{~nm}$ gapt was sandwiched between two wire waveguides of dimensions 300nm x 220nm, as shown in Figure 12. 


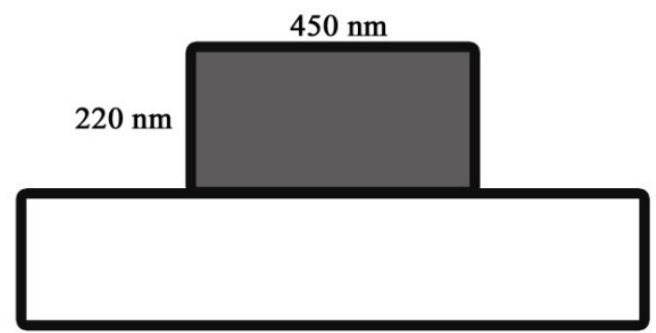

Wire Waveguide

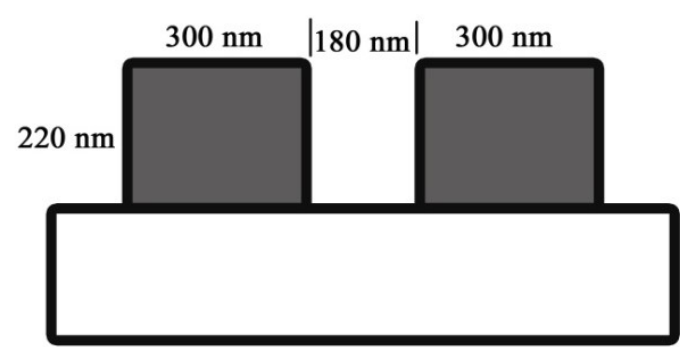

Slot Waveguide

Figure 12: Cross section of the wire waveguide and slot waveguide designs implemented.

The MRR and RAMZI sensors were implemented with DC and MMI couplers. Due to fabrication limitations, the gap in the DC was chosen to be $200 \mathrm{~nm}$ with the corresponding coupling length of $4 \mu \mathrm{m}$, while the MMI coupler design was based on the design from [59] with a rectangle dimensions $3 \mu \mathrm{m} \times 9.2 \mu \mathrm{m}$ and a center-to-center distance between the input/output ports of $1 \mu \mathrm{m}$. With these dimensions the cross power coupling ratio, $|\kappa|^{2}$ of the DC was expected to be 0.05 at $1510 \mathrm{~nm}$ and for MMI coupler the coupling ratio was expected to be 0.6 . With these cross coupling ratios the transmission coefficient of RAMZI device will match the loss in the ring near 1510nm. As shown in Figure 13, the corresponding expected power coupling losses are $0.05 \mathrm{~dB}$ and $3 \mathrm{~dB}$ for the DC and MMI coupler respectively.
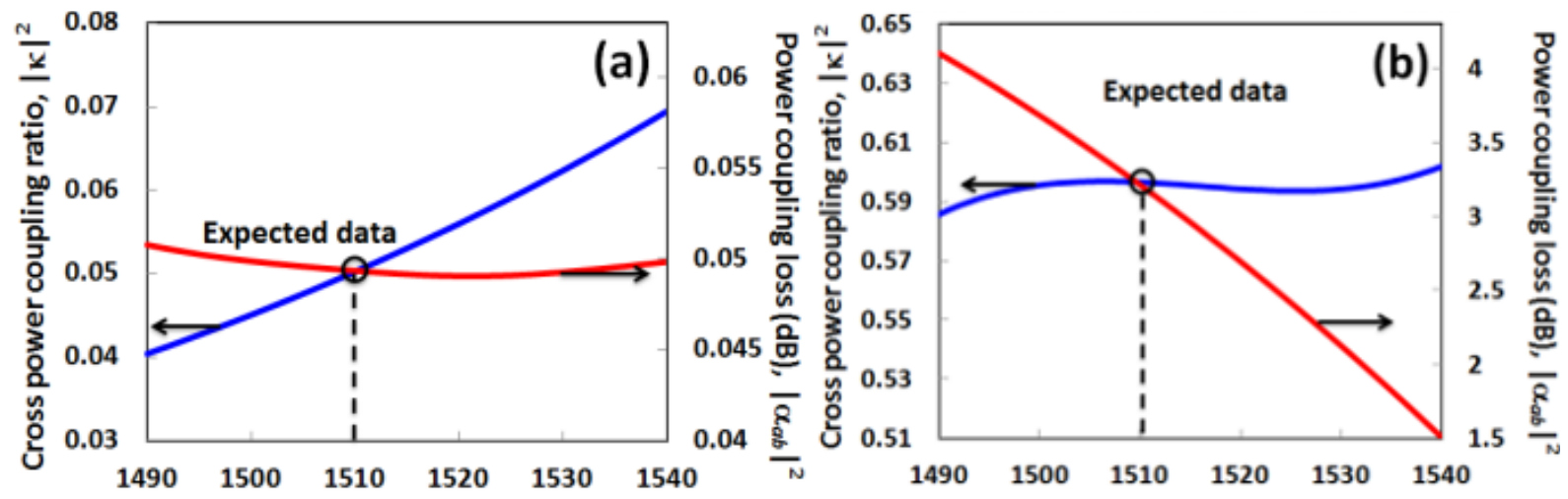

Figure 13: FDTD Simulated power cross coupling ratios (blue) and power coupling loss (red) as a function of wavelength for (a) DC coupler and (b) MMI coupler 
Two ring radiuses were required to be implemented due to the different waveguide designs. The rings ideally should be as small as possible to increase the FSR which allows for a larger measurement ranges. However, with the small radius, the bending loss increases in the devices which will result in lower Q-factors. For the wire waveguide a radius of $6 \mu \mathrm{m}$ was chosen and the slot waveguide has higher bending losses so a radius of $20 \mu \mathrm{m}$ was chosen to minimize these losses. RAMZI devices with similar $\Delta L$ (arm length difference) were chosen. For the wire waveguide devices $\Delta L=12.05 \mu \mathrm{m}$ and $\Delta L=13.14 \mu \mathrm{m}$ for DC- and MMI-coupled devices were chosen respectively. For the slot waveguide an arm length difference of $\Delta L=13.08 \mu \mathrm{m}$ and $\Delta L=13.14 \mu \mathrm{m}$ for DC and MMI-coupled devices respectively were used. The wire waveguide designs can be seen in Figure 14 and the sensors designs for the slot waveguides can be seen in Figure 15.

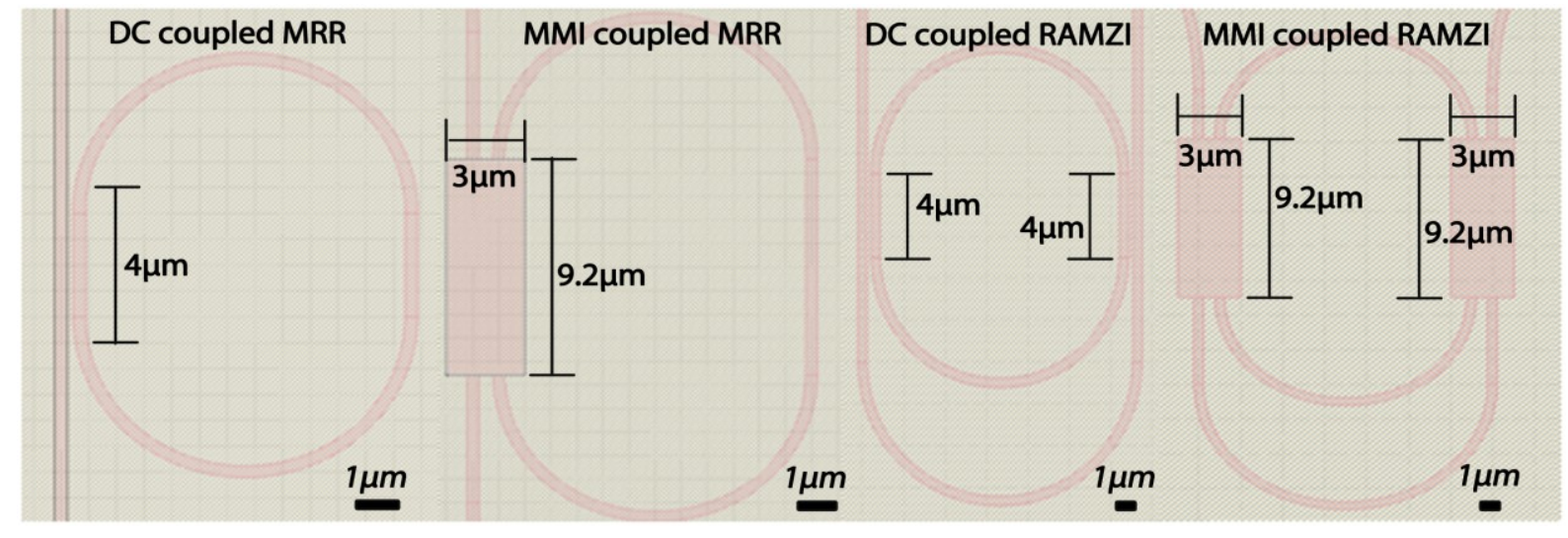

Figure 14: MRR and RAMZI wire waveguide sensor designs; all with a radius of $6 \mu m$ 


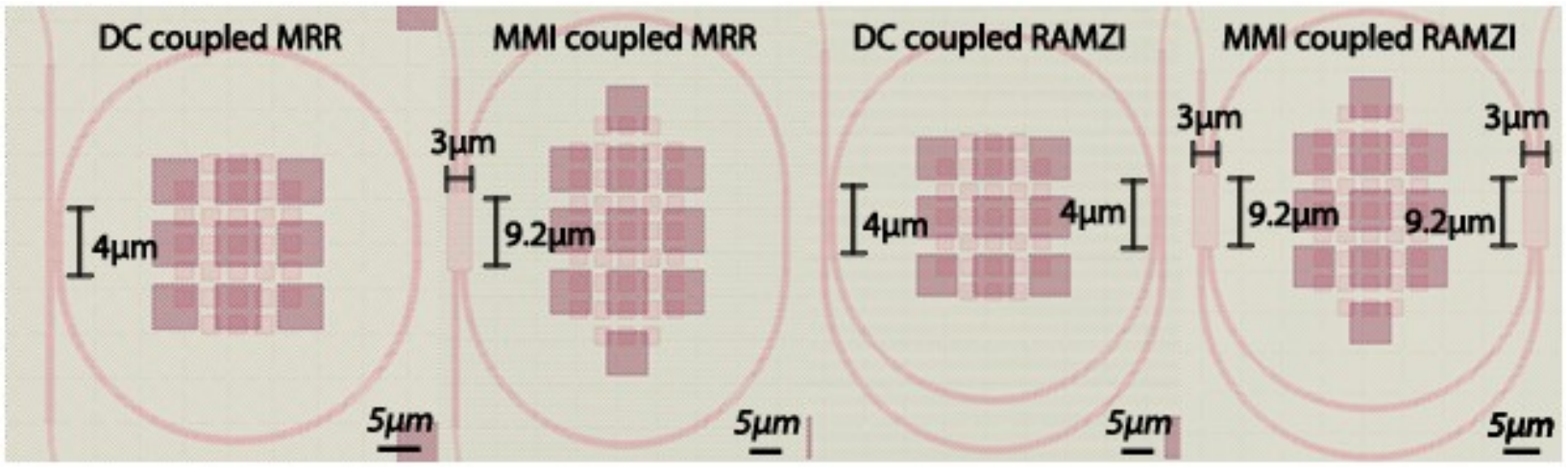

Figure 15: MRR and RAMZI slot waveguide sensor designs with a radius of $20 \mathrm{~mm}$

\subsection{Fabrication Tolerance Simulations}

The fabrication facility used has a fabrication tolerance of $\pm 20 \mathrm{~nm}$. To determine the effects of this tolerance on the coupling coefficients of the two coupler designs we will examine the couplers and coupling coefficients using Lumerical's MODE Solutions varFDTD simulator. This simulator collapses the $3 \mathrm{D}$ geometry down to a $2 \mathrm{D}$ set of effective indices to be used to solve the 2D finite-difference time-domain problem. The reduction of the waveguide into a $2 \mathrm{D}$ structure is accomplished using two different methods; a variation procedure based on Hammer and Ivanova [63] and a procedure based on the reciprocity theorem described by Snyder and Love [64]. The key assumption behind this method is that there no coupling between different slab modes in the vertical structure of the device. This is the case for our devices since our waveguide support less than two vertical modes and allows for FDTD simulations with the accuracy of a 3D FDTD simulation and the speed of the 2D FDTD simulations.[65]

For the DC two parameters were assessed; the coupling length and the coupling gap. The variation in coupler length had a negligible effect on the results for both the wire waveguide and slot waveguide. The coupling coefficients for the DC at 1550nm can be seen in Table 1. As the gap increases in size the amount light coupled into the second 
waveguide decreases. For the wire waveguide the cross coupling decreases by 0.08 for every $20 \mathrm{~nm}$ increase in gap width. And for the slot waveguide the cross coupling coefficient decreases by 0.10 forever $20 \mathrm{~nm}$ increase in gap width.

Table 1: Simulated Coupling coefficient for the DC with wire waveguides and slot waveguides

\begin{tabular}{|l|l|l|}
\hline \multicolumn{2}{|l|}{ Wire Waveguide } \\
\hline Gap Width & Through & Cross \\
\hline $180 \mathrm{~nm}$ & 0.70 & 0.30 \\
\hline $200 \mathrm{~nm}$ & 0.78 & 0.22 \\
\hline $220 \mathrm{~nm}$ & 0.83 & 0.17 \\
\hline
\end{tabular}

\begin{tabular}{|l|l|l|}
\hline \multicolumn{2}{|l|}{ Slot Waveguide } \\
\hline Gap Width & Through & Cross \\
\hline $180 \mathrm{~nm}$ & 0.26 & 0.65 \\
\hline $200 \mathrm{~nm}$ & 0.36 & 0.55 \\
\hline $220 \mathrm{~nm}$ & 0.47 & 0.45 \\
\hline
\end{tabular}

If we look at the ideal gap width of $200 \mathrm{~nm}$ the results of the coupling coefficients were unexpected. The slot waveguide which is thought to have higher confinement than the wire waveguide should have lower cross coupling. However the slot waveguide showed a cross coupling coefficient over two times that of the wire waveguide. To validate our results we can look at the mode electric profiles of both waveguides to see evanescent field for both devices. The results can be seen in Figure 16. From this, we can see that at $200 \mathrm{~nm}$ distance from the edge of the wire waveguide there is a relative electric field intensity of 0.1 and for the slot waveguide there is a relative field intensity of 0.15 . This would agree with the simulated coupling coefficients. The larger evanescent field interaction results in larger cross coupling coefficients. 

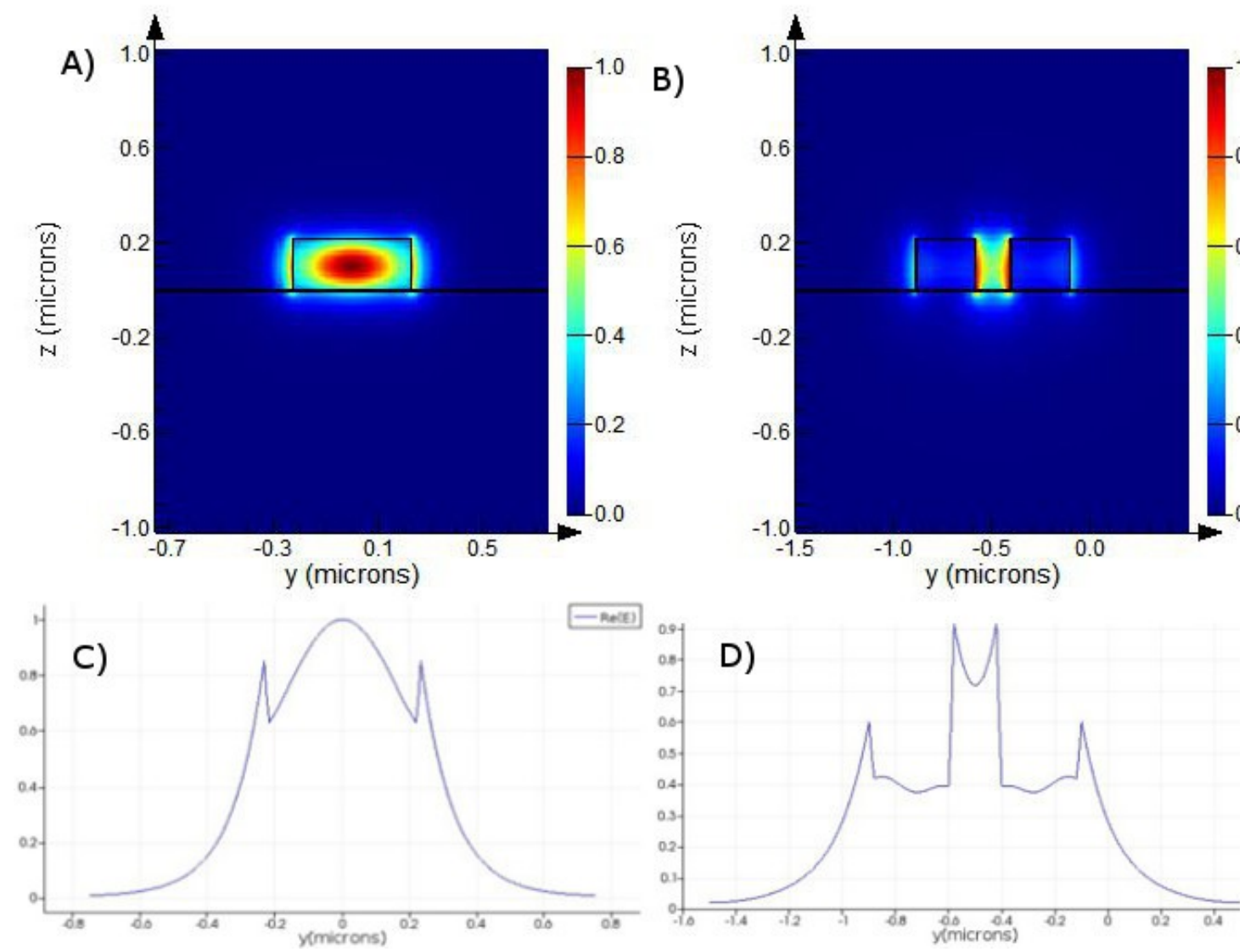

Figure 16: Simulated Electric field profiles; A) Electric field profile for the wire waveguide B)

Electric Field profile for the Slot Waveguide C) Cross section of the electric field profile for the wire waveguide along $z=0.11 \mathrm{~nm}$ D) Cross section of the electric field profile for the slot waveguide along $\mathrm{z}=0.11 \mathrm{~nm}$

For the MMI coupler there are other variables that affect the performance; the misalignment of the input and output waveguides and the width and length of the MMI coupler. The misalignment of the input and output waveguides had a negligible effect on coupling coefficients, with the through coupling and cross coupling coefficients at $1550 \mathrm{~nm}$ being 0.44 and 0.34 respectively for the wire waveguide devices and 0.30 and 0.53 for the slot waveguide devices. For the variation in coupling coefficient at $1550 \mathrm{~nm}$ of the different widths and lengths of the wire waveguide can be seen in Table 2 and for 
the slot waveguides in Table 3. From these we can observe that the variation in geometry has little affect on the through coupling coefficient; the through coupling coefficient for both wire and slot waveguide only varying by 0.03 between the two geometry extremes. The cross coupling coefficient varies more significantly with geometry; the variation of the coupler length has little impact on the cross coupling coefficient. For wire waveguide the variation results in a change of the coupling coefficient less than 0.01 and for the slot waveguide it results in a change in of only 0.02 . Whereas the width has a far more significant impact on the cross coupling coefficient, with coefficient decreasing by $0.02-$ 0.03 per $20 \mathrm{~nm}$ increase in width for both waveguides.

Table 2: Simulated coupling coefficient for the MMI coupler with sire waveguides

\begin{tabular}{|l|r|r|r|r|}
\hline \multicolumn{2}{|l|}{$\begin{array}{l}\text { Through } \\
\text { Coupling } \\
\text { Coefficient }\end{array}$} & \multicolumn{3}{l|}{ Width $(\mu m)$} \\
\cline { 3 - 5 } $\begin{array}{l}\text { Congth } \\
(\mu \mathrm{m})\end{array}$ & 9.18 & 0.42 & 0.44 & 0.44 \\
\cline { 2 - 5 } & 9.20 & 0.43 & 0.44 & 0.45 \\
\cline { 2 - 5 } & 9.22 & 0.44 & 0.45 & 0.45 \\
\hline
\end{tabular}

\begin{tabular}{|l|c|c|c|c|}
\hline \multicolumn{2}{|l|}{$\begin{array}{l}\text { Cross } \\
\text { Coupling } \\
\text { Coefficient }\end{array}$} & \multicolumn{3}{|l|}{ Width $(\mu m)$} \\
\cline { 3 - 5 } & 2.98 & 3.00 & 3.02 \\
\hline Length & 9.18 & 0.37 & 0.34 & 0.32 \\
\cline { 4 - 5 } & 9.20 & 0.37 & 0.34 & 0.32 \\
\cline { 2 - 5 } & 9.22 & 0.37 & 0.34 & 0.32 \\
\hline
\end{tabular}

Table 3: Simulated coupling coefficient for the MMI coupler with slot waveguides

\begin{tabular}{|l|r|r|r|r|}
\hline \multicolumn{2}{|l|}{$\begin{array}{l}\text { Through } \\
\text { Coupling }\end{array}$} & \multicolumn{3}{|l|}{ Width $(\mu m)$} \\
\cline { 3 - 5 } \begin{tabular}{l} 
Coefficient \\
\cline { 2 - 4 }$(\mu \mathrm{m})$
\end{tabular} & 2.98 & 3.00 & 3.02 \\
\cline { 2 - 5 } & 9.20 & 0.3 & 0.31 & 0.3 \\
\cline { 2 - 5 } & 9.22 & 0.29 & 0.3 & 0.3 \\
\hline
\end{tabular}

\begin{tabular}{|l|c|c|c|c|}
\hline \multicolumn{2}{|l|}{$\begin{array}{l}\text { Cross } \\
\text { Coupling } \\
\text { Coefficient }\end{array}$} & \multicolumn{3}{|l|}{ Width $(\mu m)$} \\
\cline { 3 - 5 } & 2.98 & 3.00 & 3.02 \\
\hline Length & 9.18 & 0.55 & 0.52 & 0.49 \\
\cline { 3 - 5 } & 9.20 & 0.56 & 0.53 & 0.49 \\
\cline { 2 - 5 } & 9.22 & 0.57 & 0.53 & 0.49 \\
\hline
\end{tabular}


From the simulations we observe that the MMI coupler is far less susceptible to fabrication errors than the DC coupler. The cross coupling coefficient of the DC coupler for the was 2.6 to 3.3 times more sensitive to coupler geometry than the MMI coupler, and the through coupling coefficient was of the DC was 8 to 10 times more sensitive to coupler geometry variations than the MMI coupler.

\subsection{Fabrication}

The devices were fabricated by OpSIS/A*STAR's IME foundry through CMC Microsystems. The platform is based on $248 \mathrm{~nm}$ deep UV lithography which allows for features sizes as small as $180 \mathrm{~nm}$. The devices were fabricated on a SOI wafer with a $220 \mathrm{~nm}$ silicon top film and a $2 \mu \mathrm{m}$ buried oxide layer, with a full $220 \mathrm{~nm}$ etch used to define the waveguides and inverse taper for coupling.

A $1.5 \mathrm{~mm} \times 2.5 \mathrm{~mm}$ space is available for the fabrication of the devices. Within this space 42 MRR and RAMZI devices were placed as well as 16 reference waveguides. The reference waveguides were of a variety of designs to determine the waveguide loss, coupling loss, bending loss, and wire to slot waveguide butt coupling losses. The MRR and RAMZI devices have three different radius $2 \mu \mathrm{m}, 6 \mu \mathrm{m}$, and $20 \mu \mathrm{m}$ with the $2 \mu \mathrm{m}$ and $6 \mu \mathrm{m}$ devices being wire waveguide based and the $20 \mu \mathrm{m}$ devices slot waveguide based. The four device designs (MRR with DC and MMI couplers, and RAMZI with DC and MMI couplers) for each radius of micro ring resonator. To accommodate for the fabrication each device design was implemented with varying coupler geometries and MZI lengths. For the sensing applications the devices require air top cladding. This was accomplished with a sensing window etched in to the silica top cladding. 


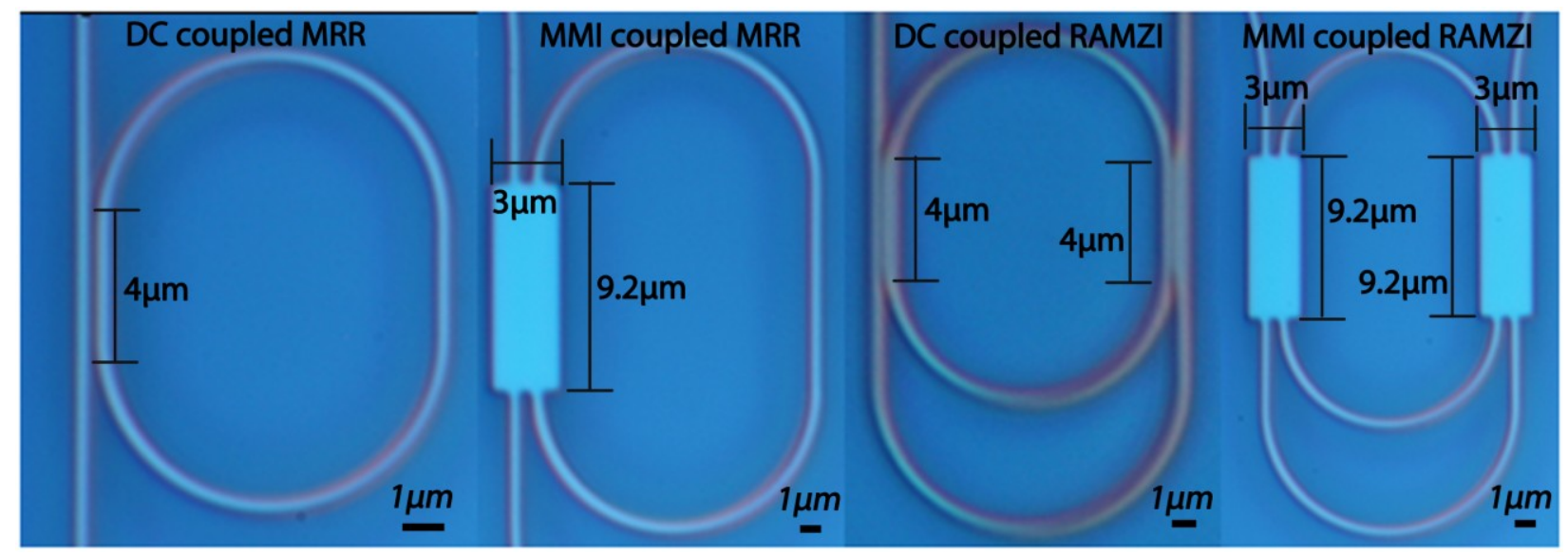

Figure 17: Fabricated MRR and RAMZI wire waveguide sensors; all with a radius of $6 \mu \mathrm{m}$

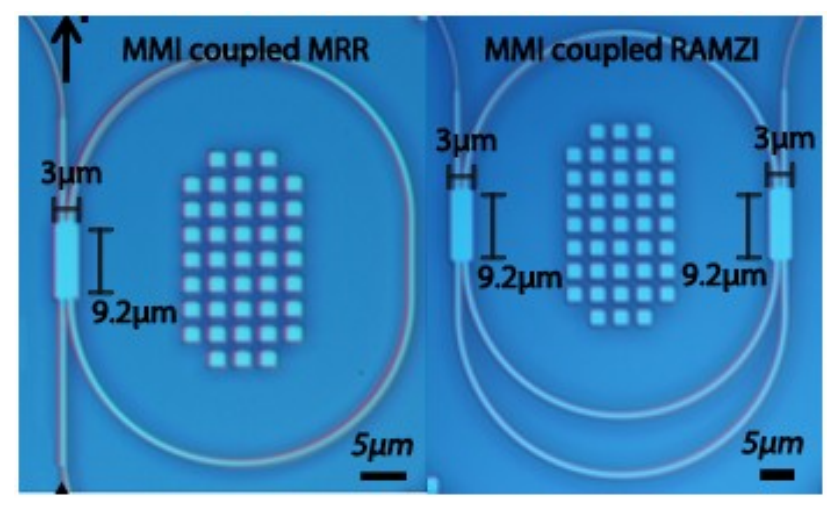

Figure 18: Fabricated MRR and RAMZI slot waveguide sensors with a radius of $20 \mu \mathrm{m}$,

Due to the size mismatch of a single mode optical fiber $(10 \mu \mathrm{m})$ and single mode silicon waveguide $(500 \mathrm{~nm} \times 220 \mathrm{~nm}$ rectangular cross section), the ability to couple light from one to the other is limited. To overcome this limitation two techniques were used, as illustrated in Figure 19; reducing the modal distribution in the optical fiber and the use of waveguide input and output couplers. The modal distribution in the optical fiber was reduced by the use of a tapered and lensed fiber. With these fibers the optical mode can be focused down to a spot size of $2-6.5 \mu \mathrm{m}$ in diameter [60]. An effective waveguide input/output coupler design is that of the inverse nanotaper, first demonstrated by $\mathrm{V}$. R. Almeida et al [61]. The device is a short taper with a nanometer sized tip at the edge of 
the chip. The small size of the tip causes the mode of the waveguide core to become delocalized which increases the mode overlap between the fiber and the waveguide. The combination of the tapered and lensed optical fiber and inverse taper coupler has been reported to achieve a coupling loss as low as $1 \mathrm{~dB}$ [62], making it an excellent choice for our design. Our tapered coupler is connected to the $450 \mathrm{~nm}$ x $220 \mathrm{~nm}$ wire waveguide and terminates with geometry of $200 \mathrm{~nm} \times 220 \mathrm{~nm}$ at the facet, with the length of the taper being approximately $0.5 \mathrm{~mm}$.

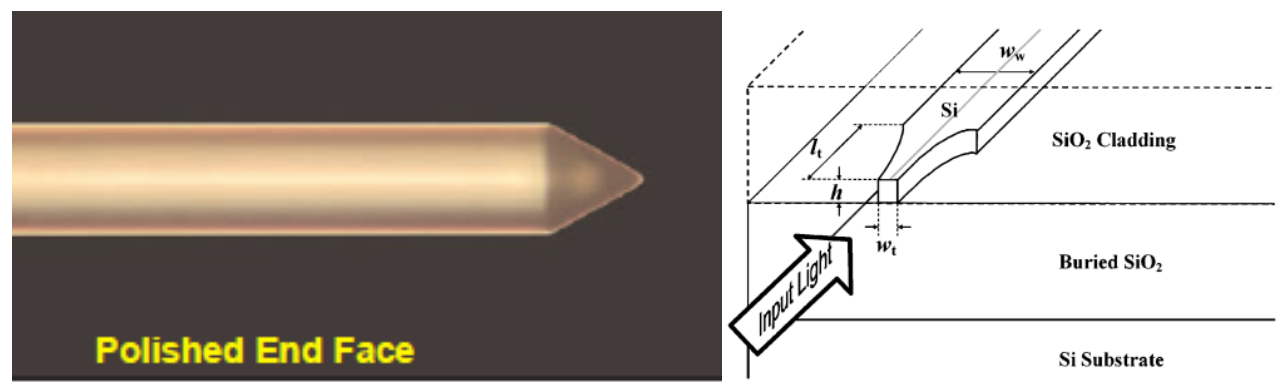

Figure 19: Left: $\mathrm{OZ}$ optics tapered and lensed optical Fiber[60], Right: Inverse nanotaper 


\section{Chapter: Device Testing}

This chapter includes a discussion on the experimental apparatus used for testing our device, the procedures of the testing, and the results of the testing. The discussion of the results of the devices are separated into discussion of the spectral characteristics of the devices and discussion of the sensing performance.

\subsection{Experimental Apparatus}

The devices were measured at Carleton University with the experimental setup consisted of three major components; a tunable laser source and detector, tapered and lensed optical fibers and a mechanical stage for alignment. For our setup a Luna Optical Vector Analyzer (OVA) model 5000 was used. This device acts as both a tunable laser source and detector in the $\mathrm{C}$ and $\mathrm{L}$ band. The OVA is capable of measuring insertion loss and polarization dependent loss with a resolution of $1.6 \mathrm{pm}$, an accuracy of $\pm 1.5 \mathrm{pm}$ and a dynamic loss range of $60 \mathrm{~dB}$ over a wavelength range of $1525 \mathrm{~nm}$ to $1610 \mathrm{~nm}$, making it an ideal source and detector for our measurements.

The tapered and lensed fibers for coupling were manufactured by $\mathrm{OZ}$ optics and were polarization maintaining. The mechanical stages used for alignment were Thorlabs Nanomax 341M 3 axis stages with stepper motor actuators and closed-loop piezoelectric actuators. Three stages were used, two for the input and output fibers and a third stage for the chip to sit on. All three were controlled by a program written in Labview which was edited for this setup. The stepper motor actuators provide $4 \mathrm{~mm}$ travel with a minimum step size of $60 \mathrm{~nm}$ and the piezoelectric actuators have a $20 \mu \mathrm{m}$ of travel with a minimum resolution of $5 \mathrm{~nm}$ plus a feedback loop to compensate for any drift. The stages offered sufficient accuracy for coupling to the silicon waveguides. 
Due to the small size of the waveguide the coupling is highly susceptible to vibrations of the test bench. The location of the experimental setup was on the fifth floor of the Minto building and we found the noise level due to vibrations to be $20-30 \mathrm{~dB}$. This required the optical table to be isolated from the vibrations of the laboratory. Newport pneumatic vibration isolators were used. With these the noise due to vibration was reduced to a value no larger than $2 \mathrm{~dB}$. This was deemed sufficient to able to easily identify the resonant peaks.

The apparatus can be seen in Figure 20 which shows the three mechanical stages with two of the stages holding the input and output lensed fiber and the third holding the chip mount for the testing. The microscope and lamp were required for alignment purposes.

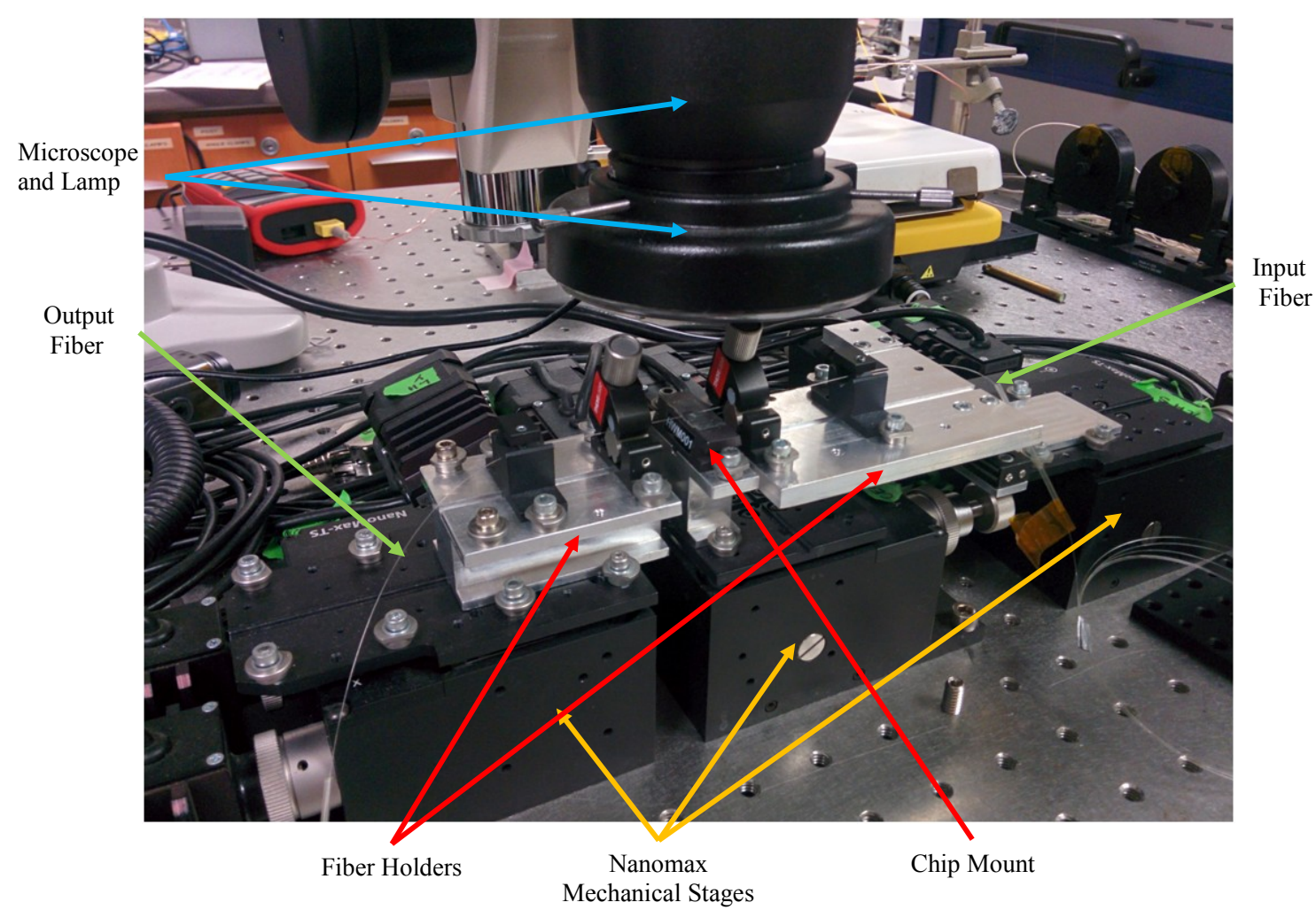

Figure 20: Experimental apparatus 


\subsection{Experimental Results}

The experimental results are broken into two sections. First we will examine the characteristics of the MRR and RAMZI devices with air cladding, and second, the bulk index sensing capabilities of the sensors using different concentration levels of sucrose.

For the characterization of the devices we examined the spectral characteristics of the devices by identifying the FSR (or quasi-FSR in case for the RAMZI devices), ER, FWHM, and Q-factor for the devices. The tests were all performed with air cladding, i.e. no liquid on the surface of the device. One exception is that of the slot waveguide with DC, where we were unable to observe resonance with air cladding so a DI water top cladding was applied. A Lorentzian curve was fitted to the data to determine the resonant peak wavelengths.

For the bulk index sensing, tests of the device sucrose solution with varying concentrations were used. The solutions were made with deionized (DI) water mixed with sucrose by mass. The concentrations were varied from $0 \%$ to $6 \%$ in $1 \%$ increments, which corresponds to a linear increase of the index of refraction from 1.3330 to 1.3418 [66]. Between tests the device chip was cleaned with DI water. Before each sensing

measurement, a reference measurement was performed to compensate for possible temperature drift that may have occurred during the testing process.

Another important factor that defines a sensor is the detection limit, the minimum detectible shift the device can measure. Detection limit is equal to the minimum detectable wavelength shift divided by the sensitivity of the device. The minimum detectable wavelength shift is dependent on the minimum spectral resolution of the detector but also on the quality factor and extinction ratio of the resonant peak and the 
measurement noise on the spectrum. These factors affect the uncertainty of the fitted Lorentzian peak. In [43] De Vos approximates the minimum measureable shift to be $1 / 15^{\text {th }}$ that of the FWHM. This method was strictly empirical. White and Fan discuss a more theoretical bases for determining the detection limit of the device in [67]. They use spectral variations, amplitude variation, and thermal variations of waveform to determine the detection limit. White and Fan propose the minimum resolution of the device to be 3 standard deviations of the noise produced by the device and errors introduced by the system of measurement. This includes the noise related to thermal drift, amplitude related noise and the spectral resolution of the system given by the following equation,

$$
3 \sigma=3 \sqrt{\sigma_{a m p}^{2}+\sigma_{T}^{2}+\sigma_{\text {spectral }}^{2}}
$$

Where $\sigma_{a m p} \approx F W H M /\left(4.5(S N R)^{0.25}\right)$, where FWHM is the full width half maximum of the device, $S N R$ is the signal to noise ratio in linear units, $\sigma_{T}$ is the error associated with thermal drift, and $\sigma_{\text {spectral }}$ is the error associated with the minimum resolution of the detector used.

From Eq. (43) the sensitivity of the devices is dependent on the wavelength of the peak. For our measurements span a range of $85 \mathrm{~nm}$, around centered on $1565 \mathrm{~nm}$ this results in a $5 \%$ difference in the sensitivities. However for most of the devices the resonant peak used for measurement was between 1530-1560nm, which would correspond to $2 \%$ difference in the sensitivities measured at $1530 \mathrm{~nm}$ versus $1560 \mathrm{~nm}$. For this reason the peak for which the shift was measured was chosen as follows; a major peak was selected for the RAMZI device then a peak for the MRR equivalent device was selected with approximately the same resonant wavelength. 


\subsubsection{Devices Characteristics - DC- and MMI-based devices using wire}

\section{waveguides}

For the MRR with a radius of $6 \mu \mathrm{m}$, the transmission spectrum of the DC-coupled

wire

waveguide

device

can

be

seen

in
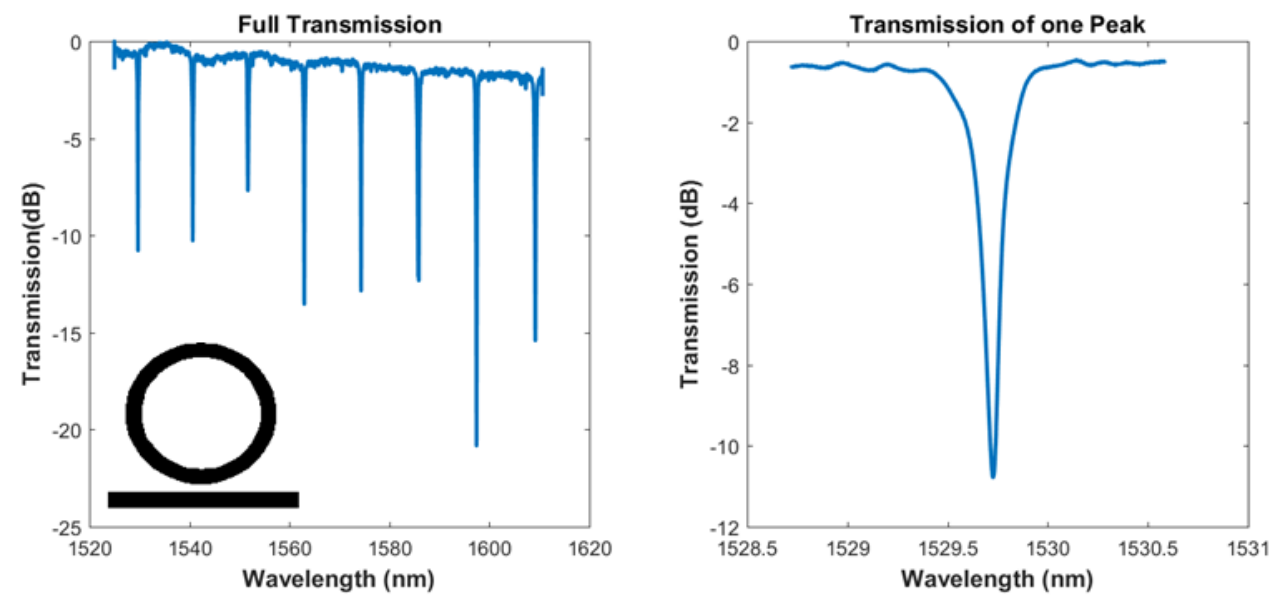

Figure 21, with a magnified transmission of a single resonant peak. At the resonant peak of $1529.7 \mathrm{~nm}$, the device had a FSR of $10.95 \mathrm{~nm}$, an ER of $10.32 \mathrm{~dB}$, FWHM of $0.1423 \mathrm{~nm}$ and Q-factor of 10750.
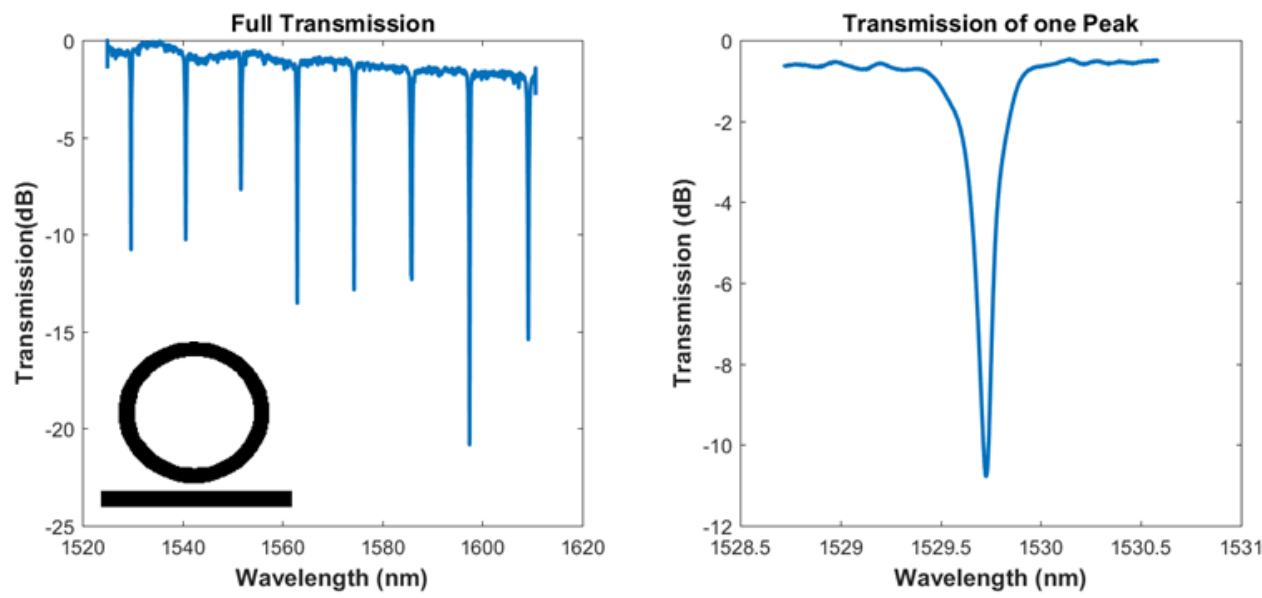

Figure 21: Transmission spectrum and transmission of a single peak for a DC-coupled MRR with wire waveguides. 
For the MRR with a radius of $6 \mu \mathrm{m}$ and MMI coupler the transmission spectrum of the device and the transmission of a single peak can be seen in Figure 22. For the resonant peak at $1532.1 \mathrm{~nm}$ the device had a FSR of $9.41 \mathrm{~nm}$, an ER of $11.32 \mathrm{~dB}$, FWHM of $1.455 \mathrm{~nm}$ and Q-factor

of 1050. Compared to
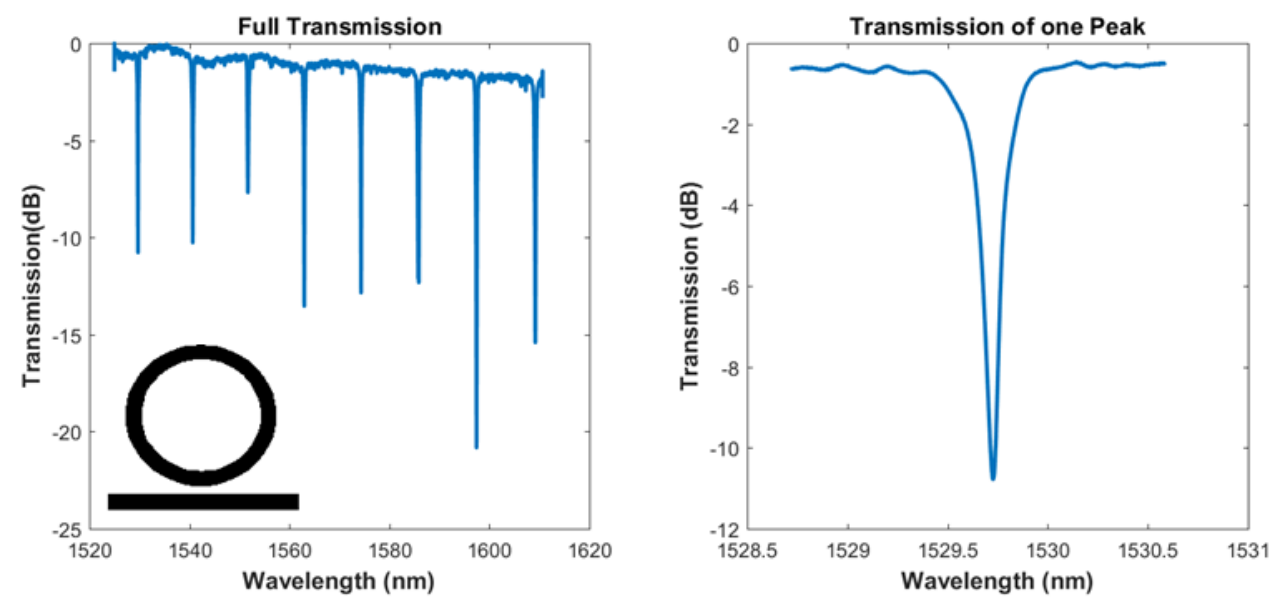

Figure 21, the MMI-coupled MRR has a FWHM and Q-factor one tenth that of the DCcoupled MRR. This is the expected result: looking at the equations for FWHM Eq. ( 26 ) and Q-factor Eq. ( 29 ) we can see an increase in loss causes an increase to the FWHM and a decrease to the Q-factor. The FSR is also reduced $1.54 \mathrm{~nm}$ this again is logical, the peak is a higher wavelength and the optical path length of the MMI-coupled MRR is larger than that of the DC-coupled MRR. 

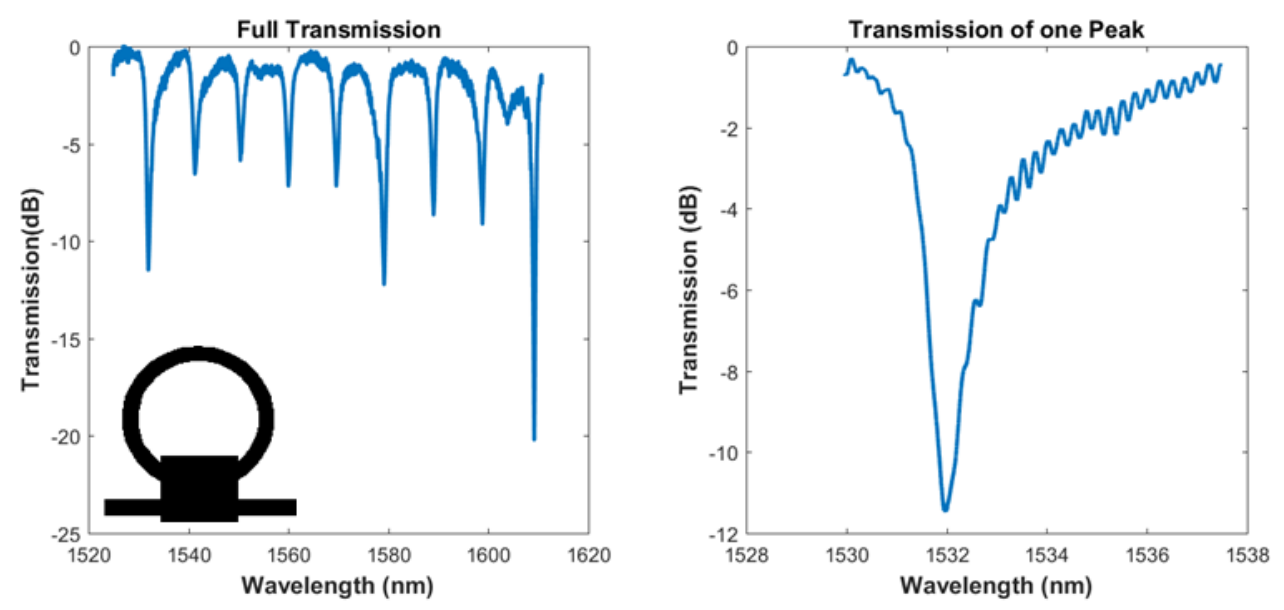

Figure 22: Transmission spectrum and transmission of a single peak for the MMI-coupled MRR with wire waveguides.

Figure 23: Transmission spectrum and transmission of a single peak for the DCcoupled RAMZI device with wire waveguides. The difference in arm lengths is $\boldsymbol{\Delta} \boldsymbol{L}=$ $12.05 \mu \mathrm{m}$. shows the transmission spectrum of the DC-coupled wired-based RAMZI with a radius of $6 \mu \mathrm{m}$, and a difference in arm length of $\Delta L=12.05 \mu \mathrm{m}$. For the resonant peak at $1534.1 \mathrm{~nm}$, the device has an ER of $7.94 \mathrm{~dB}$, FWHM of $0.2870 \mathrm{~nm}$ and Q-factor of 5346. The normal FSR is $11.26 \mathrm{~nm}$. It appears that every $4^{\text {th }}$ resonant peak has a noticeable larger ER with major peaks at $1534.1 \mathrm{~nm}, 1568.1 \mathrm{~nm}$, and $1602.9 \mathrm{~nm}$, which correspond to a quasi-FSR of $34.0 \mathrm{~nm}$. Comparing this to the results from the DC-coupled 

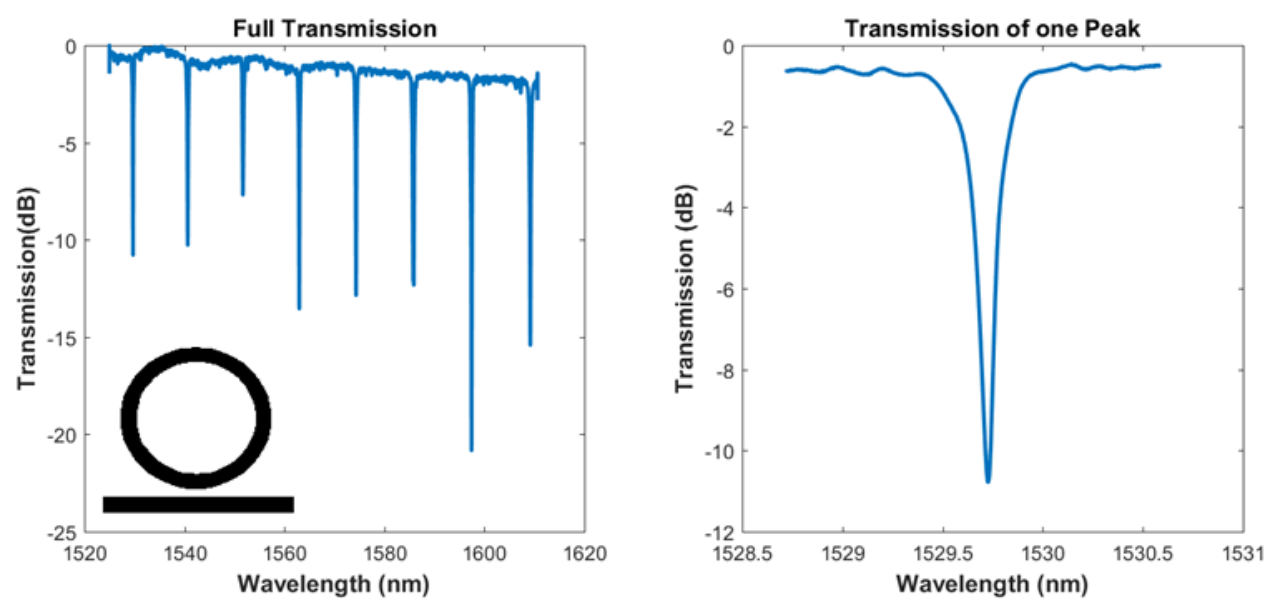

Figure 21, we see the RAMZI device increases the FSR by 3.1 times. Using Eq. ( 27 ) and the results of DC-coupler MRR to approximate a group index and then using this value in ( 39 ) we get a theoretical Quasi FSR of 34.5nm. Comparing this theoretical value to the measured value we find a $1.4 \%$ discrepancy in values, which is not signficant. Therefore the FSR increase is in agreement with the theoretical results.
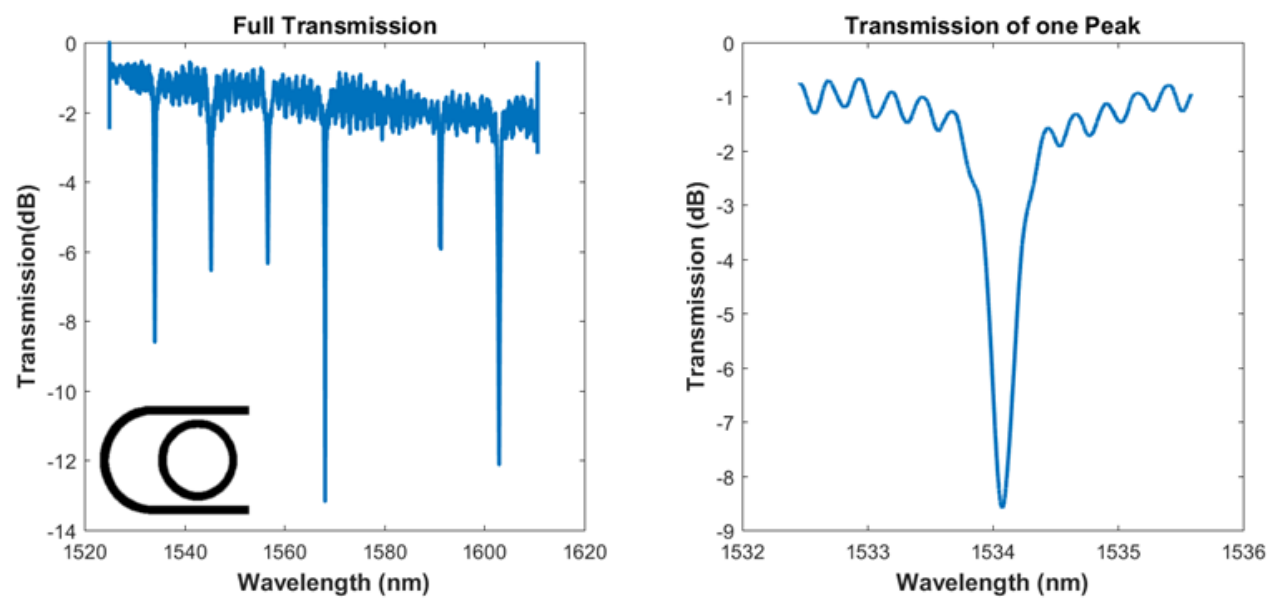

Figure 23: Transmission spectrum and transmission of a single peak for the DC-coupled RAMZI device with wire waveguides. The difference in arm lengths is $\Delta L=12.05 \mu \mathrm{m}$.

Figure 23 shows the transmission spectrum of the MMI-coupled wire-based RAMZI with a radius of $6 \mu \mathrm{m}$, and a difference in arm length of $\Delta L=13.14 \mu \mathrm{m}$. For the 
resonant peak at $1531.8 \mathrm{~nm}$ the device has an ER of $8.26 \mathrm{~dB}$, FWHM of 1.948nm and Qfactor of 786.2. The device has a normal FSR of $11.26 \mathrm{~nm}$, and a quasi-FSR of $27.03 \mathrm{~nm}$. The calculated quasi-FSR using the previously mentioned method (Eq. (27) and (39)) is $26.98 \mathrm{~nm}$, which is in agreement with our experimental results. It is noted that the quasiFSR is smaller than the $34.0 \mathrm{~nm}$ of the DC-coupled RAMZI device due to lower wavelength of the resonant peak and the difference in arm lengths is $1.09 \mathrm{~nm}$ larger. However, when we compare this to the results of the MMI-coupled MRR with a FSR of $9.41 \mathrm{~nm}$ we see an increase of 2.87 times.
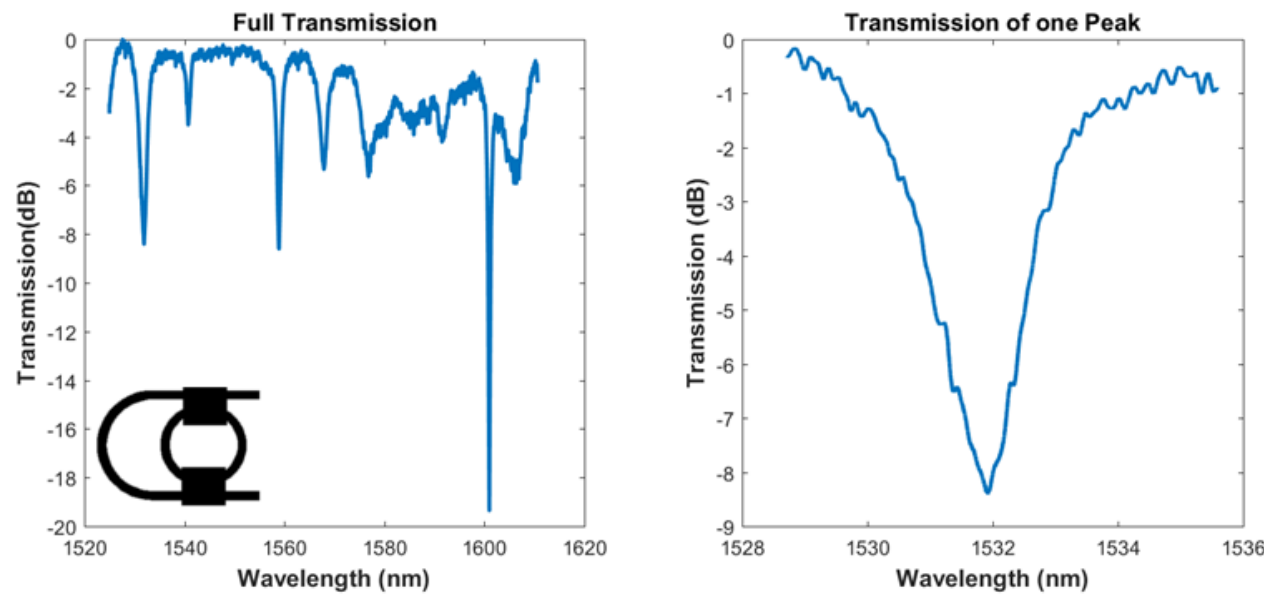

Figure 24: Transmission spectrum and transmission of a single peak for the MMI-coupled RAMZI device with wire waveguides. The difference in arm length is $\Delta L=13.14 \mu \mathrm{m}$

From Figure 23 and Figure 24, we can also observe that the change in FSR is not dependent on the coupler design, but on the ratio of the optical path length in the MRR to the difference in arm length of the RAMZI device. That is, for the DCs the FSR to quasiFSR ratio is 3.1 and the ratio of lengths is 3.1; and for the MMI couplers the FSR ratio is 2.87 and the ratio of lengths is 2.87 . This is the expected result. If we compare the FSR equations for the two devices; Eq. ( 27 ) and (( 39 ), they only vary by the length term, $L$ for the MRR and $\Delta L$ for the RAMZI device. 
Examining the effect on the $\mathrm{Q}$ factor of the RAMZI devices we can see that the $\mathrm{Q}$ factor is reduced, for the DCs the Q factor is reduced by a factor of 2.0 and for the MMI couplers the Q factor is reduced by 1.3 times. This reduction is most likely due to the increased loss through the micro ring and the variation in the coupling coefficients due to fabrication errors. We can conclude that the MMI couplers are more robust design with the Q-factor of the devices being reduced by a smaller factor than that of the DC devices.

From Figure 21 through Figure 24 we can see that the MMI coupler performs better over the full range of wavelengths when compared to the DC coupler. In the DCcoupled designs in Figure 21 and Figure 23 the maximum transmission drops by $2 \mathrm{~dB}$ from $1525 \mathrm{~nm}$ to $1610 \mathrm{~nm}$. In the MMI-coupled designs in Figure 22 and Figure 24 we see the maximum transmission remains relatively constant over the wavelength span. This result is not large however for the slot waveguide this difference is more evident.

\subsubsection{Devices Characteristics - DC- and MMI-based devices using slot waveguides}

Now we focus on devices that use slot waveguides. The transmission spectrum of the DC-coupled MRRs with a radius of $20 \mu \mathrm{m}$ is shown in Figure 25. For the resonant peak at $1531.1 \mathrm{~nm}$ the device had a FSR of $4.76 \mathrm{~nm}$, an ER of $10.94 \mathrm{~dB}$, FWHM of 1.096nm and Q-factor of 1397.0. 

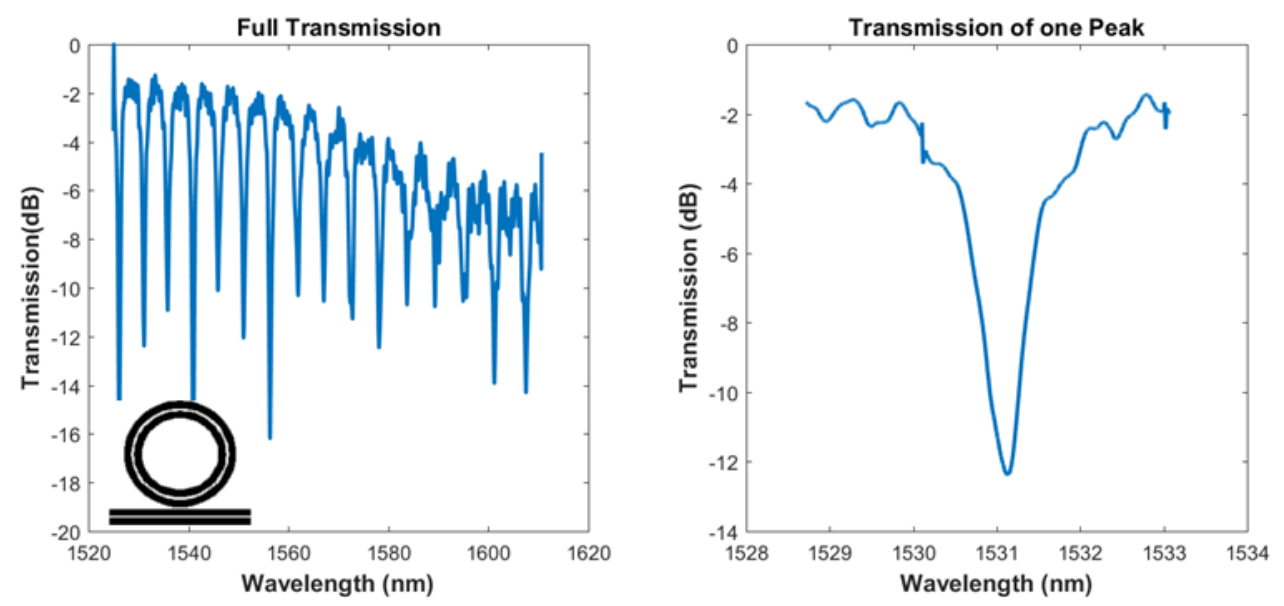

Figure 25: Transmission spectrum and transmission of a single peak for the DC-coupled MRR device with slot waveguides

The transmission spectrum of the MMI-coupled slotted MRR with a radius of $20 \mu \mathrm{m}$ is shown in Figure 26. For the resonant peak at $1532.1 \mathrm{~nm}$ the device had a FSR of $4.11 \mathrm{~nm}$, an ER of $15.92 \mathrm{~dB}, \mathrm{FWHM}$ of $0.8919 \mathrm{~nm}$ and Q-factor of 1718. Compared to DC-coupled device presented in Figure 25, the MMI-coupled device has a Q-factor 1.23 times larger.
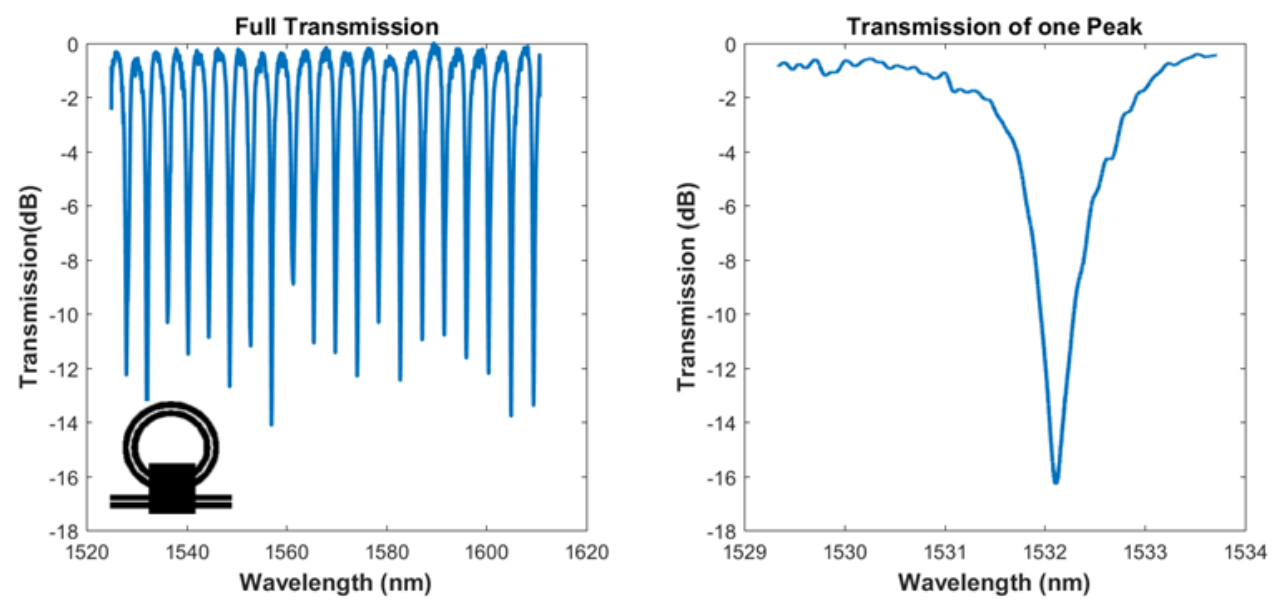

Figure 26: Transmission spectrum and transmission of a single peak for the MMI-coupled MRR device with slot waveguides. 
For the DC-coupled RAMZI with a radius of $20 \mu \mathrm{m}$ and a difference in arm length of $\Delta L=13.08 \mu \mathrm{m}$, the transmission spectrum of the device and the transmission of a single peak can be seen in Figure 27. At the resonant peak at $1537.0 \mathrm{~nm}$ the device has an ER of $34.65 \mathrm{~dB}, \mathrm{FWHM}$ of $.574 \mathrm{~nm}$ and Q-factor of 2678 . For this device the quasi-FSR of the device was clearly observed $\mathrm{n}$ to be $24.2 \mathrm{~nm}$ and with a normal FSR of $4.79 \mathrm{~nm}$. We can compare this to the FSR of the equivalent MRR of 4.76nm (in Figure 25). The quasiFSR is larger by 5.08 times. We can use the same technique from above to determine the theoretical quasi-FSR for this device configuration based on the MRR equivalent. In this way we find a theoretical quasi-FSR of $46.1 \mathrm{~nm}$. This value disagrees with the measured value. The cause of this discrepancy has not been identified.
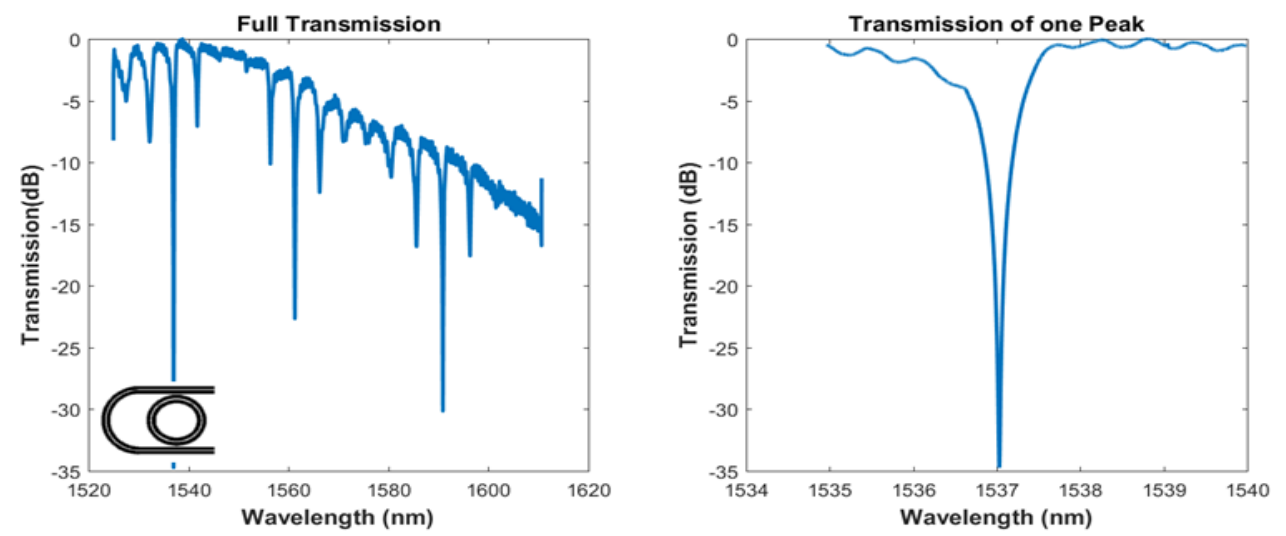

Figure 27: Transmission spectrum and transmission of a single peak for the DC-coupled RAMZI device with slot waveouide with an arm lenoth difference of $\Delta I .=13.08$ um

The transmission spectrum of the MMI-coupled RAMZI with a radius of $20 \mu \mathrm{m}$ and a difference in arm length of $\Delta L=13.14 \mu \mathrm{m}$ can be seen in Figure 28. At the resonant peak at $1557.7 \mathrm{~nm}$ the device has an ER of $14.65 \mathrm{~dB}$, FWHM of $3.046 \mathrm{~nm}$ and Q-factor of 511.4. We estimate that the normal FSR is $4.41 \mathrm{~nm}$ and a quasi-FSR is $43.7 \mathrm{~nm}$. Comparing the FSR to that of the MRR equivalent with a FSR of $4.11 \mathrm{~nm}$ we oberve the FSR increased by 10.6 times. The theoretical quasi-FSR based on the MRR results yields 
a quasi-FSR of $40.6 \mathrm{~nm}$, which agrees reasonably well with the previous FSR increase seen in the wire waveguides.
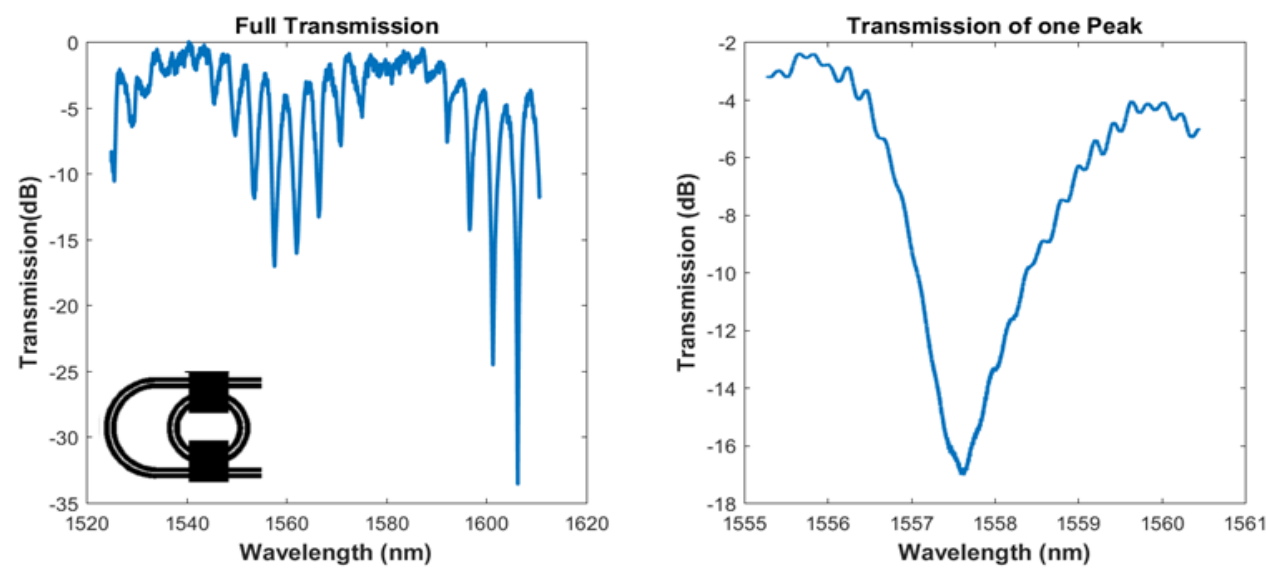

Figure 28: Transmission spectrum and transmission of a single peak for the MMI-coupled RAMZI with slot waveguides. The arm length difference of $\Delta L=13.14 \mu \mathrm{m}$

The results of testing MMI coupled designs with the slot waveguides agree with the results found previously for the wire waveguide designs. The FSR increase is in agreement with theory. As well we can see the ratio of optical path length of the MRR to the difference in arm length determines the increase in FSR. The ratio of lengths is 10.6 and ratio of FSR is 9.6.

For the MMI-coupled RAMZI design with slot waveguides we can see the Qfactor is reduced by a factor 3.35 which is a more significant loss than seen in the wire waveguides. The reduced Q-factor is most likely due to the $k_{a d}$ not meeting the critical coupling condition.

The DC-coupled RAMZI design with slot waveguides appears to be an outlier with the ratio of lengths and FSRs in disagreement with the theory; that is, the ratio of lengths is 9.6 and the FSR ratio is 5.08. In addition, the Q-factor of the device is higher than that of the MRR design by a factor of 2.13 . This discrepancy could be due to the fact 
the same coupler design was used for the wire waveguide and slot waveguide devices, Since the design is more sensitive to the geometry changes compared to the MMI coupler this result could be expected.

We can compare the FSR of slot waveguide devices to that of the wire waveguide devices using Eq. (27), and Eq. (39). For the MRR devices we find the FSR for the slot waveguide devices is 2.3 times lower than the wire waveguide equivalent devices, and the optical path length is roughly 3.3 times longer for the slot waveguide devices. Since the resonant wavelengths are approximately equal we can find the group index of the slot waveguide to be 0.69 times that of the wire waveguide. For the two MMI-coupled RAMZI devices we calculated the group index of the slot waveguide to be 0.62 times the group index of wire waveguide. This agrees with the calculation for the MRR devices. However for the DC-coupled device we calculated the group index of the slot waveguide to be 1.29 times that of the wire waveguide. This discrepancy is again most likely to be due to the DC coupler design.

Again we can see the MMI-coupled designs are less sensitive to wavelength variations and perform well over the full spectrum tested. For the DC-coupled designs in Figure 25 and Figure 27 the maximum transmission occurs between 1530nm and 1540nm and then drops $6 \mathrm{~dB}$ and $15 \mathrm{~dB}$ for the MRR and RAMZI designs respectively. In the MRR design we see the Q-factor of the devices drops past 1560nm. From Figure 26 and Figure 28 we see that for the MMI-coupled devices the performance does not vary over the wavelength span. The MRR design has a maximum transmission which is constant over the wavelength span while for the RAMZI device the maximum transmission varies over the range of measurements. The variation of the RAMZI devices is due to the MZI 
effects in the devices; the peaks in maximum transmission are roughly the same distance as that of the quasi-FRS.

The characteristic parameters of all the devices are summarized in Table 4. From this we can see the RAMZI device offers a larger FSR by a factor of 3 to 10, depending on the specific design. This however does appear to come at the cost of a lower Q-factor by a factor of 1.3-3.3 times. One exception to this is the DC-coupled MRR and RAMZI with slot waveguides, where the Q-factor is doubled. It is clearly demonstrated that in general, the RAMZI device increases the FSR and thus will increase the total sensing range of the device.

Table 4: Device Characteristic Parameters

\begin{tabular}{|c|c|c|c|c|c|c|c|}
\hline \multicolumn{3}{|l|}{ Device } & \multirow[t]{2}{*}{$\lambda_{\text {res }}(\mathrm{nm})$} & \multirow{2}{*}{$\begin{array}{l}\text { FSR } \backslash \text { Quasi- } \\
\text { FSR (nm) }\end{array}$} & \multirow{2}{*}{$\begin{array}{l}\text { ER } \\
\text { (dB) }\end{array}$} & \multirow{2}{*}{$\begin{array}{l}\text { FWHM } \\
(\mathbf{n m})\end{array}$} & \multirow{2}{*}{$\begin{array}{l}\text { Q- } \\
\text { Factor }\end{array}$} \\
\hline Waveguide & Design & & & & & & \\
\hline \multirow{4}{*}{$\begin{array}{c}\text { Wire } \\
\text { Waveguide }\end{array}$} & & MRR & 1529.7 & 10.95 & 10.32 & 0.142 & 10750 \\
\hline & & MRR & 1532.1 & 9.41 & 11.32 & 1.455 & 1050 \\
\hline & & RAMZI & 1534.1 & 34.0 & 7.94 & 0.287 & 5346 \\
\hline & & RAMZI & 1531.8 & 27.03 & 8.26 & 1.948 & 786.2 \\
\hline \multirow{3}{*}{ Waveguide } & & MRR & 1531.1 & 4.76 & 10.94 & 1.096 & 1397.0 \\
\hline & & MRR & 1532.1 & 4.11 & 15.92 & 0.892 & 1718 \\
\hline & & & 1537.0 & 24.2 & 34.65 & 0.574 & 2678 \\
\hline
\end{tabular}




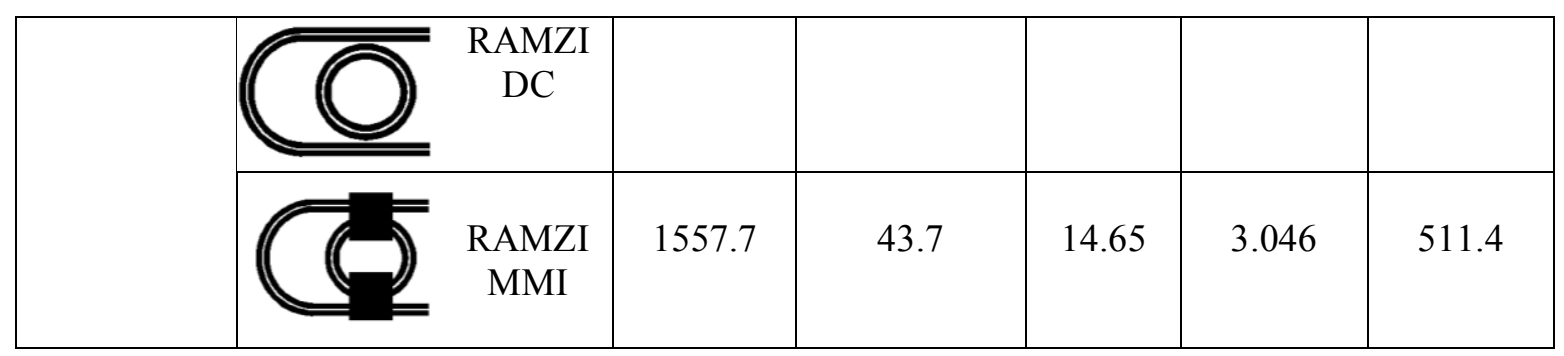

\subsubsection{Bulk Index Sensing using DC- and MMI-coupled wire waveguides}

The bulk index sensing of the MRR designs with wire waveguides with the DC and MMI couplers can be seen in Figure 29 and Figure 30 respectively. Here a liquid solution of sucrose with a linear $1 \%$ increasing concentration from $0 \%$ to $6 \%$; the refractive index increases from 1.3330 to 1.3418 . The sensitivity of the DC-coupled MRR with wire waveguides is $170.8 \pm 12.4 \mathrm{~nm} / \mathrm{RIU}$. The detection limit of the device was found to be $5.8 \times 10^{-5}$ RIU. Comparing this to the values reported in literature, De Vos et al. in [43] presented a 70nm/RIU for a wire waveguide MRR with a DC. We see an improvement in the sensitivity limit in our demonstrated devices. The sensitivity of the MMI-coupled MRR with wire waveguides is $213.6 \pm 13.1 \mathrm{~nm} / \mathrm{RIU}$, which corresponds to a detection limit in the magnitude again in the range of $4.4 \times 10^{-4}$ RIU. The sensing performance of the MMI-coupled MRR is better than that of the DC-coupled MRR sensors due to the MMI-coupled MRRs are less sensitive to the change in material than the DC-couplers. 

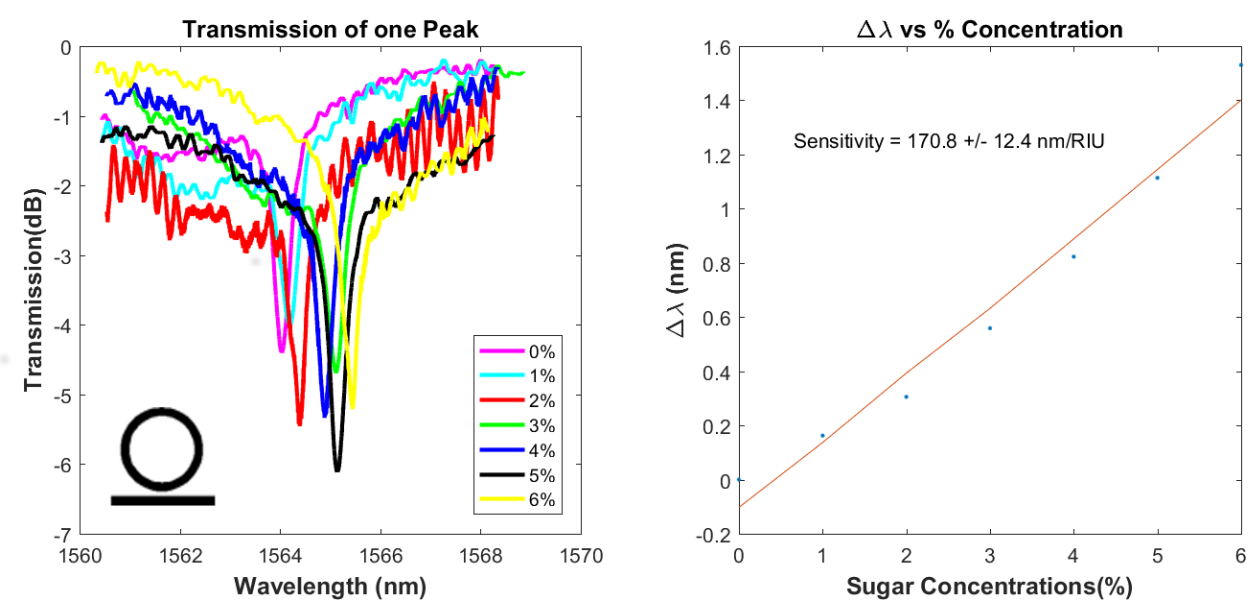

Figure 29: Transmission of One Peak for various sugar concentrations and the Change of resonant peak location vs sugar concentration for the DC-coupled MRR with wire waveguides
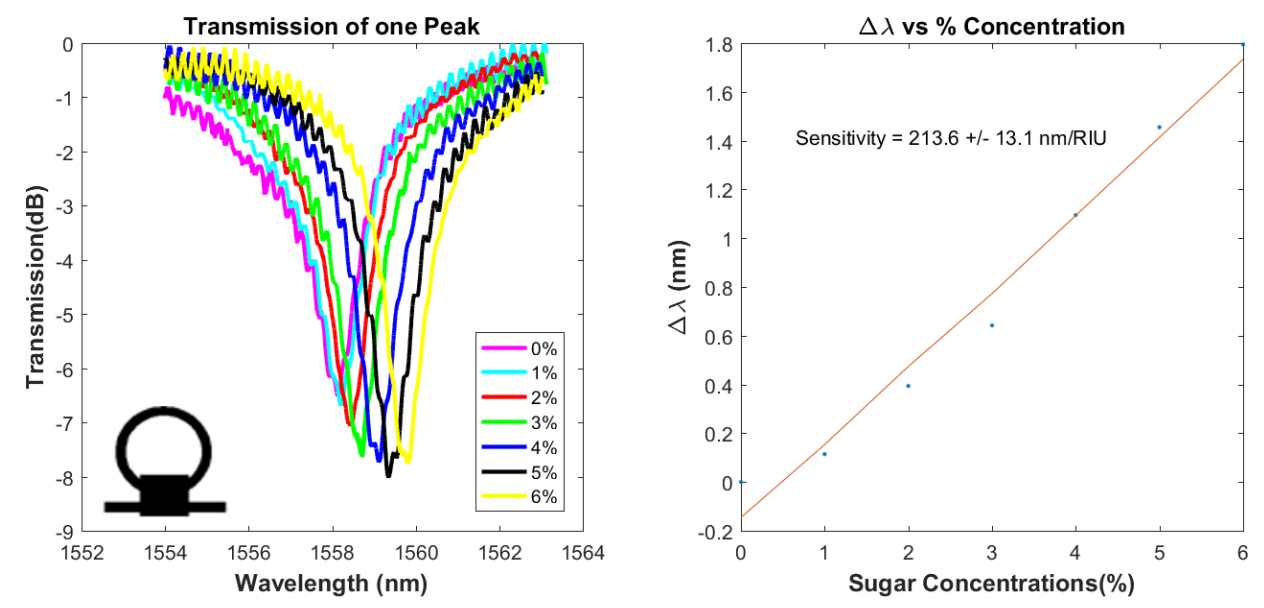

Figure 30: Transmission of One Peak for various sugar concentrations and the Change of resonant peak location vs sugar concentration for the MMI-coupled MRR with wire waveguides

The bulk index sensing of the RAMZI designs with the DC and MMI couplers can be seen in Figure 31 and Figure 32 respectively. The sensitivity of the DC-coupled RAMZI with wire waveguides is $176.0 \pm 7.9 \mathrm{~nm} / \mathrm{RIU}$ with a detection limit of $8.0 \times 10^{-5}$ RIU. The sensitivity of the MMI-coupled RAMZI with wire waveguides is $73.95 \pm 3.1 \mathrm{~nm} / \mathrm{RIU}$ with a detection limit in the magnitude again in the range of $5 \times 10^{-}$ 
${ }^{4}$ RIU. We can compare our result to that of Wang and Dai in which they reported a sensitivity for the RAMZI devices with wire waveguide and DC coupler of $111 \mathrm{~nm} / \mathrm{RIU}$. [33] We can see that our sensors have a sensitivity 1.7 times that of the Wang and Dai device. We can also see that the DC-coupled RAMZI device has an increased sensitivity than that of the MMI-coupled device. This is likely due to the DC coupler design being better optimized for the RAMZI than the MMI-coupler; the DC coupler better satisfies the phase matching conditions.
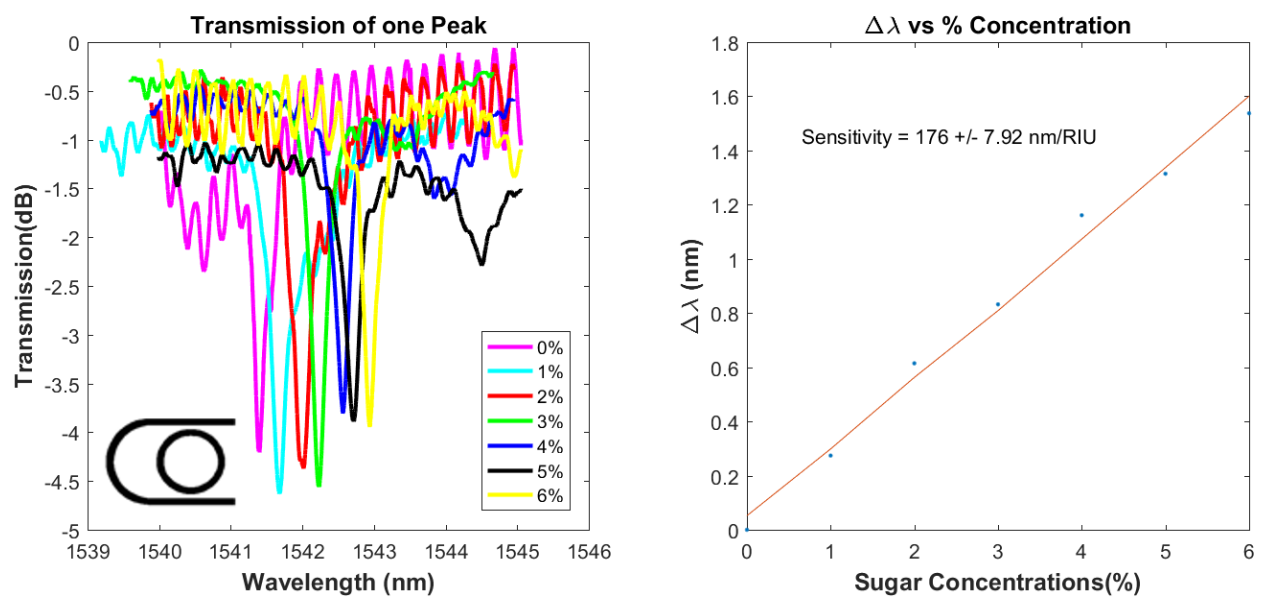

Figure 31: Transmission of One Peak for various sugar concentrations and the Change of resonant peak location vs. sugar concentration for the DC-coupled RAMZI with wire waveguides . The difference in arm length is $\Delta L=12.05 \mu \mathrm{m}$. 

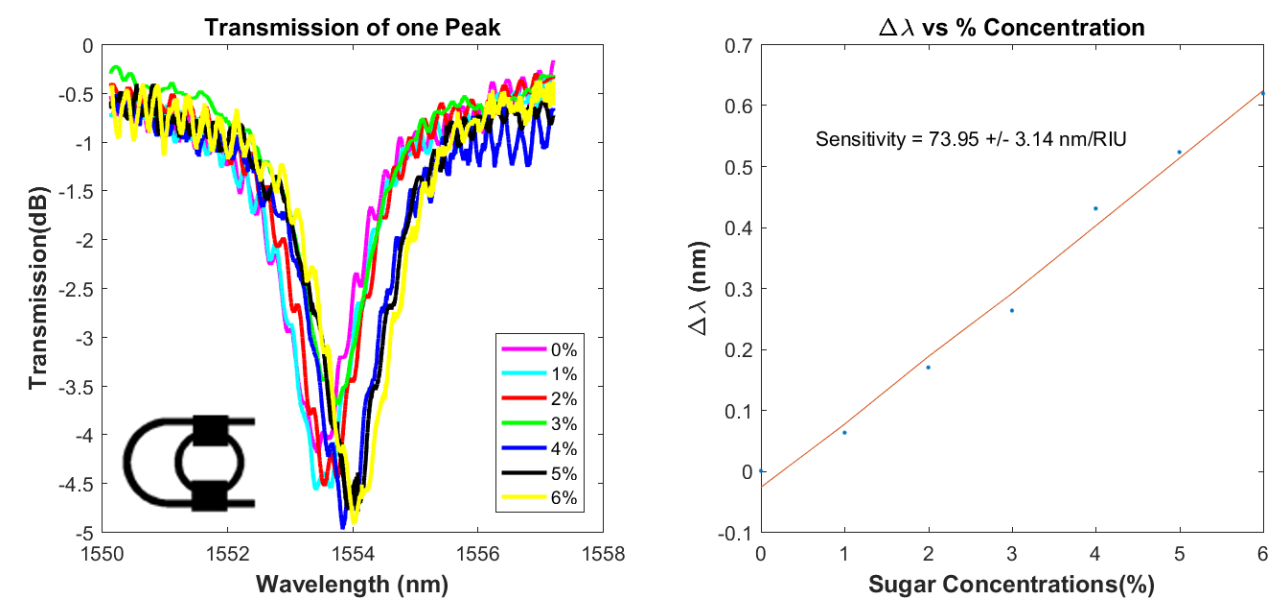

Figure 32: Transmission of One Peak for various sugar concentrations and the Change of resonant peak location vs. sugar concentration for the MMI-coupled RAMZI with wire waveguides. The difference in arm length is $\Delta L=13.14 \mu \mathrm{m}$

\subsubsection{Bulk Index Sensing using DC- and MMI-coupled slot waveguides}

The bulk index sensing of the MRR designs with slot waveguides with the DC and MMI couplers can be seen in Figure 33 and Figure 34 respectively. The sensitivity of the DC-coupled MRR with slot waveguides is $297.5 \pm 11.4 \mathrm{~nm} / \mathrm{RIU}$ with a detection limit of $1.4 \times 10^{-5} \mathrm{RIU}$. The sensitivity of the MMI-coupled MRR, on the other hand, is $66.2 \pm 3.5 \mathrm{~nm} / \mathrm{RIU}$, with a corresponding detection limit in the magnitude again in the range of $4.3 \times 10^{-4} \mathrm{RIU}$. The result of the DC device can be compared to that of the result presented in the literature. Our devices operates with a similar sensitivity (298nm/RIU) as reported in [28]. This confirms that the experimental setup employed in this thesis produces valid results although this controls are not as thorough as those described in the literature. We can see that the DC-coupled device performs significantly better than that of the MMI coupler device. This is due to the DC-coupler being a better design to operate at the concentrations examined. 

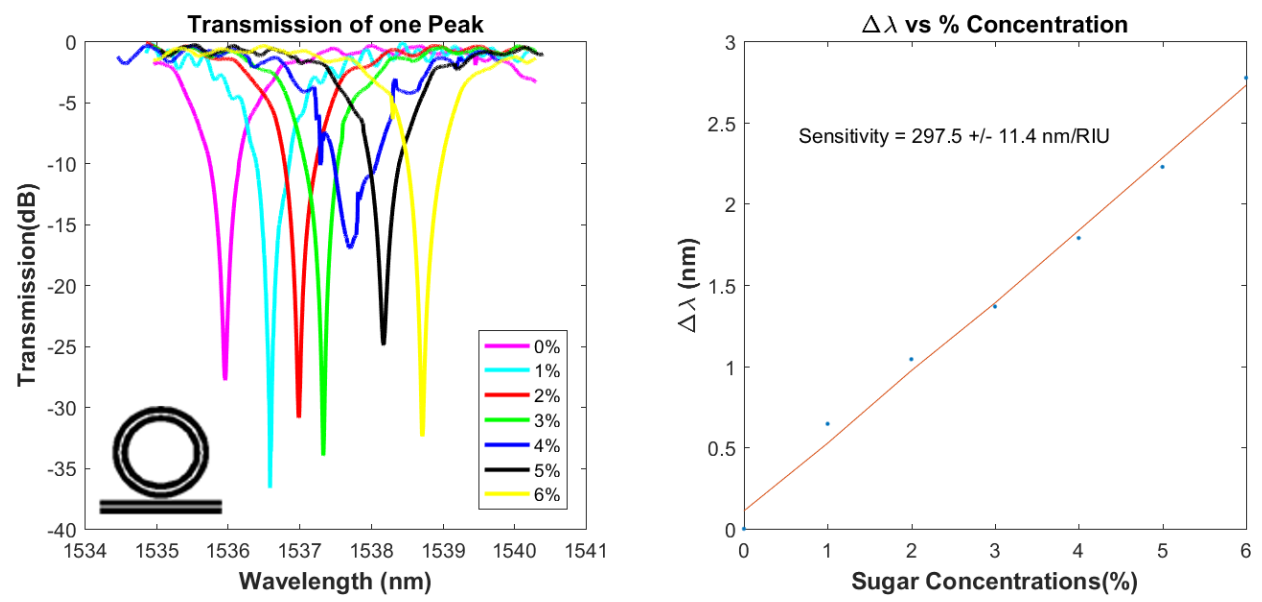

Figure 33: Transmission of One Peak for various sugar concentrations and the Change of resonant peak location vs. sugar concentration for the DC-coupled MRR with slot waveguides
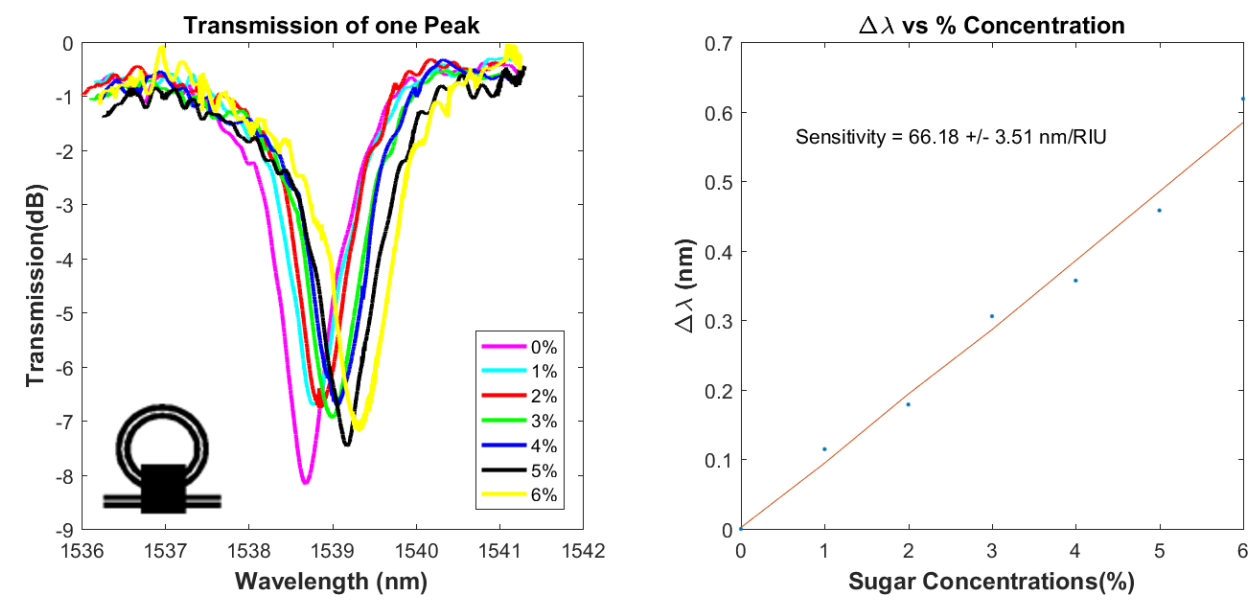

Figure 34: Transmission of One Peak for various sugar concentrations and the Change of resonant peak location vs. sugar concentration for the MMI-coupled MRR with slot waveguides

The bulk index sensing of the RAMZI designs with slot waveguides with the DC and MMI couplers can be seen in Figure 35 and Figure 36 respectively. The sensitivity of the DC-coupled RAMZI with slot waveguides is $217.6 \pm 7.4 \mathrm{~nm} / \mathrm{RIU}$ with a detection limit in the magnitude of $6.0 \times 10^{-5} \mathrm{RIU}$; while the sensitivity of the MMI-coupled RAMZI is $176.0 \pm 12.8 \mathrm{~nm} / \mathrm{RIU}$ with a detection limit in the magnitude again in the range of $5.1 \times 10^{-}$ 
${ }^{5}$ RIU. Again we see the DC-coupled device has a higher sensitivity than MMI-coupled device. This is due to the reason previously stated for the MRR devices.

The inconsistencies in the sensitivity enhancement from the RAMZI devices could be attributed to the coupling regimes for each device. The MRR based sensor operating in the overcoupled regime will have a reduced sensitivity and for maximum sensitivity the undercoupled regime is ideal [68]. The a ring-coupled MZI device achieves higher sensitivity enhancement when operating in the overcoupled regime [69]. Although our RAMZI device design is not the same as the device in [69], the theory presented in that reference agrees with our findings. The DC-coupled devices would be operating in the undercoupled regime which would result in the single MRR operating with optimal sensitivity and the MZI/RAMZI having reduced sensitivity. And MMIcoupled devices would operate in the overcoupled regime, which would cause an MRR device to have low sensitivity and the RAMZI devices to see a sensitivity enhancement. If we consider the results presented in section 4.2 we actually see that the DC with the slot waveguide has a coupling coefficient similar to that of the MMI couplers. The coupling coefficient would not be the cause of the inconsistencies. However I do not think the presented simulations reflect the coupler that was fabricated. As previous stated, during testing no resonance was seen for the device with air cladding. This indicates that no coupling was occurring whereas in simulation we see the strong coupling occurring. The inconsistencies in the sensitivity enhancement the RAMZI device is could be the subject for further investigation. 

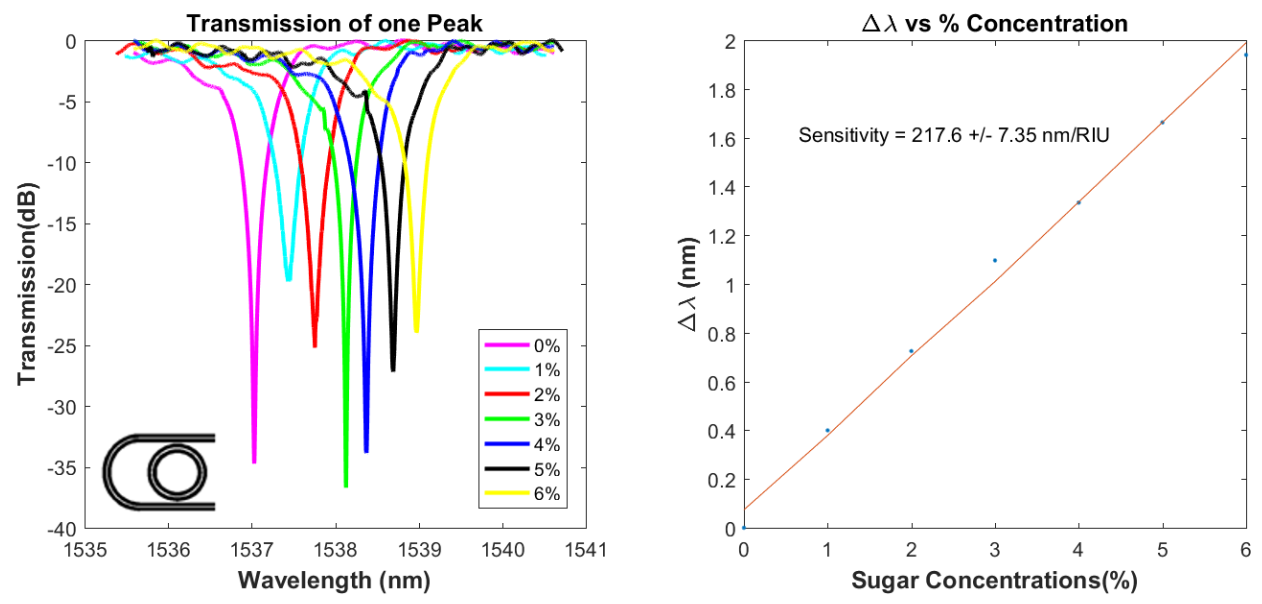

Figure 35: Transmission of One Peak for various sugar concentrations and the Change of resonant peak location vs sugar concentration for the DC-coupled RAMZI with slot waveguides. The difference in arm length is $\Delta L=13.08 \mu m$
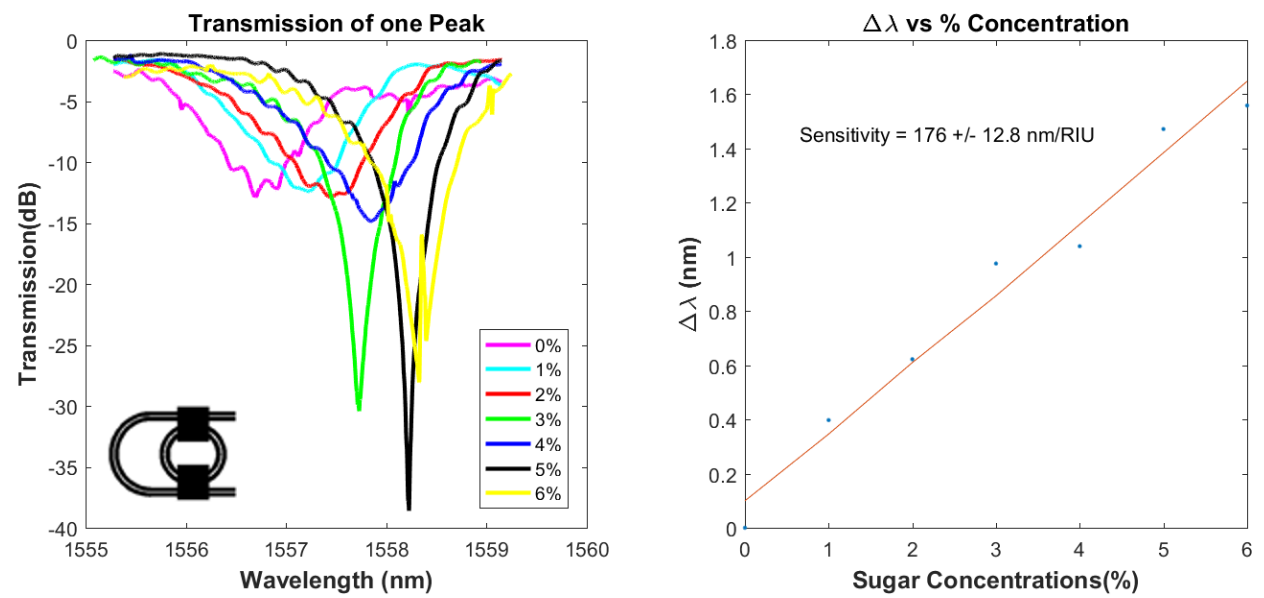

Figure 36: Transmission of One Peak for various sugar concentrations and the Change of resonant peak location vs sugar concentration for the MMI-coupled RAMZI with slot waveguides. The difference in arm length is $\Delta L=13.14 \mu \mathrm{m}$

The summarized results on all the sensors tested are listed in Table 5. In our bulk index sensing experiments, the slot waveguides offer an increased sensitivity by a factor of between 1.07 and 2.2, with an exception of one outlier (the MMI-coupled MRR). We 
also find the detection limit increases on average by an order of magnitude. The bulk index sensing ranges for all the configurations are also summarized in Table 5. The MRR with slot waveguides has the high sensitivity and small FSR; which results in a maximum change refractive index of $0.016 \mathrm{RIU}$ between two measurements for them to remain distinguishable. The RAMZI device with wire waveguides however demonstrated the largest sensing range; due its lower sensitivity and high FSR. It gives a maximum refractive index shift of 0.366RIU for two measurements to remain distinguishable. Both the RAMZI slot waveguides show good performance in both sensitivity and measurement range; with the DC based device having a sensing range of 0.119 RIU and $0.248 \mathrm{RIU}$ for the MMI based device.

Table 5: Device Sensitivities and Detection Limits

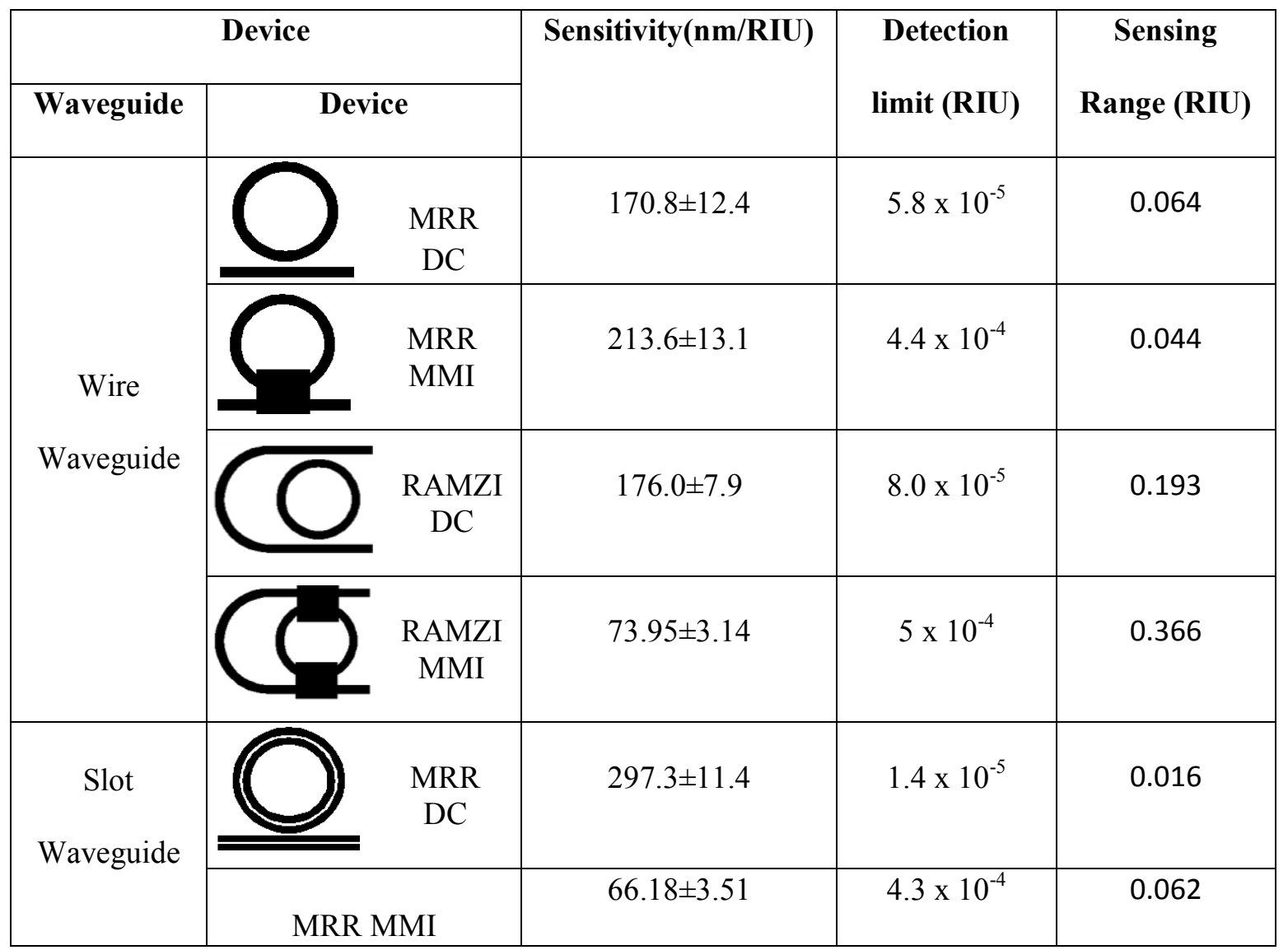




\begin{tabular}{|l|l|l|l|l|}
\hline & & & \\
\hline \\
\hline
\end{tabular}




\section{Chapter: Conclusion}

This thesis covers the design and testing of SOI micro ring resonators (MRRs) and ring assisted Mach-Zehnder interferometer (RAMZI) designs for the use as biological and chemical sensors. The effects of coupler design on sensing performance were also observed comparing the directional coupler (DC) and multimode interference (MMI) coupler. Finally the sensitivity enhancement of the slot waveguide when compared to the wire waveguide was observed. Both the implementation of RAMZI device with slot waveguide and the effects of coupler design on sensing performance have not previously been demonstrated.

The RAMZI performed as expected by increasing the FSR by a factor of 3 to 10 times depending on the coupler and waveguides used. However this was at the cost of a reduction of the Q-factor, with the RAMZI device reducing the Q-factors by 1.3 to 3.3 times. Further investigation into the design parameters in the couplers could find a method to mitigate the reduction in Q-factor. An increase in Q-factor was witnessed in the DC-coupled slot waveguide design, indicating the potential of the RAMZI devices. It was also seen that the DC-coupled devices achieve a higher Q-factors but are more sensitive to wavelength variations6 than the MMI-coupled devices. Because of this the DC-coupled devices would be ideal for application where high sensitivity is required and MMI-coupled devices would be ideal for applications where a large range of measurement are required.

The bulk index sensing capabilities of the MRR and RAMZI devices were measured using a sucrose solution. The concentration of the solution was varied from $0 \%$ to $6 \%$, resulting in a linear index of refraction change from 1.3330 to 1.3418 . The DC- 
coupled devices were found to have superior sensitivity and the MMI-coupled devices were found to be less susceptible to fabrication variations, and less wavelength dependent than the DC-coupled devices. The RAMZI implementation increased the index of refraction detection range, with comparable sensitivities. The slot waveguides were found to increase the sensitivity by 1.07 to 2.28 times that of the wire waveguide designs. The optimal configuration for sensing was found to be DC-coupled MRR with slot waveguide; with a sensitivity of $297.3 \mathrm{~nm} / \mathrm{RIU}$ and a detection limit of $1.5 \times 10^{-5} \mathrm{RIU}$.

\subsection{Future Work}

In this thesis we demonstrate the advantages of the RAMZI over the conventional MRR design, however there is further work that could be done to improve the performance of the MRR and RAMZI devices. The length and gap between the waveguides of the DC couplers used for the wire waveguides and slot waveguides were identical. The coupling strengths for the two waveguide geometries were different. The difference caused the absence of the resonant peaks in the output from the slot devices with air cladding. While the devices with wire waveguides all performed well with air cladding, when liquid was introduced to the surface, the resultant resonant peaks had a significantly lower Q-factor. Further investigation in the optimization of the couplers designs for both waveguide geometries would improve the device performance for both slot and wire waveguides.

Moreover, the consistency of the measurements was difficult to achieve with the current setup. Improvements to the testing setup could result in significant improvement with accuracy as well as a reduction in the time required to make the measurements. For example, currently a reference measurement is performed each time before measurements 
to account for the thermal drift of the MRR and RAMZI device, If a thermoelectric heater/cooler were to be used the effect of thermal drift could be significantly reduced and the reference measurement would no longer be needed. Another improvement to the experimental setup would be to have a flow cell placed onto the top of the sensor chip such as done in [28]. The authors of [28] use $7 \mathrm{~mm}^{2}$ closed channel to introduce the liquid to the surface. Currently the liquid is placed onto the surface of the device and the measurement was taken as quickly as possible to avoid the effects of evaporation on the solution concentration. A flow cell would allow for better control of the concentration of the sensing liquid and allow for an easier switching between different solutions for measurements. A flow cell would also allow for the devices to be exposed to the liquid being sensed for a greater period of time which would increase the probability of the slot in the slot waveguides being filled.

Finally, the thesis project can be extended to provide the sensing of surface binding of proteins. The most commonly used protein used to test surface sensing is that of the avidin binding to biotin receptor molecules, such as demonstrated by [28]. Surface morphology study will be necessary to confirm the receptor attachment to the device surface. 


\section{References}

[1] K. Vlachos, C. Raffaelli, S. Aleksic, N. Andriolli, D. Apostolopoulos, H. Avramopoulos, D. Erasme, D. Klonidis, M. N. Petersen, M. Scaffardi, K. Schulze, M. Spiropoulou, S. Sygletos, I. Tomkos, C. Vazquez, O. Zouraraki, and F. Neri, "Photonics in switching: enabling technologies and subsystem design," J. Opt. Netw., vol. 8, no. 5, pp. 404-428, May 2009.

[2] J. Xie, "Dual Beam Laser Welding," Weld. Res., vol. 81, no. 10, pp. 223-230, 2002.

[3] G. (George) Xiao and W. J. Bock, Photonic Sensing, 1st ed. Hoboken, N.J: Wiley, John Wiley \& Sons Inc, John Wiley \& Sons, Wiley-Blackwell, 2012.

[4] BCC Research, "Optical Sensing: Technologies and Global Markets."

[5] S. E. Miller, "Integrated optics: An Introduction,” bell Syst. Tech. J., pp. 2059$2069,1969$.

[6] R. A. Soref and B. R. Bennett, "Electrooptical effects in silicon," IEEE J. Quantum Electron., vol. 23, no. 1, pp. 123-129, 1987.

[7] R. A. Soref and J. P. Lorenzo, "Single-crystal silicon: a new material for 1.3 and $1.6 \mu \mathrm{m}$ integrated-optical components," Electron. Lett., vol. 21, no. 21, pp. 953954, 1985.

[8] G. T. Reed, W. R. Headley, and C. E. J. Png, "Silicon photonics: the early years," vol. 5730, p. 1, 2005.

[9] Y. Vlasov and S. McNab, "Losses in single-mode silicon-on-insulator strip waveguides and bends.," Opt. Express, vol. 12, no. 8, pp. 1622-1631, 2004.

[10] P. Dong, N.-N. Feng, D. Feng, W. Qian, H. Liang, D. C. Lee, B. J. Luff, T. 
Banwell, a Agarwal, P. Toliver, R. Menendez, T. K. Woodward, and M. Asghari, "GHz-bandwidth optical filters based on high-order silicon ring resonators.," Opt. Express, vol. 18, no. 23, pp. 23784-23789, 2010.

[11] S. Xiao, M. H. Khan, H. Shen, and M. Qi, "Multiple-channel silicon microresonator based filters for WDM applications: erratum.," Opt. Express, vol. 15, no. 15, p. $9386,2007$.

[12] P. Dong, S. Liao, D. Feng, H. Liang, D. Zheng, R. Shafiiha, C.-C. Kung, W. Qian, G. Li, X. Zheng, A. V Krishnamoorthy, and M. Asghari, "Low Vpp, ultralowenergy, compact, high-speed silicon electro-optic modulator," Opt. Express, vol. 17, no. 25, pp. 22484-22490, Dec. 2009.

[13] P. Dong, R. Shafiiha, S. Liao, H. Liang, C. C. Kung, W. Qian, D. Feng, G. Li, X. Zheng, A. V. Krishnamoorthy, and M. Asghari, "Broadly tunable high speed silicon micro-ring modulator," 2010 IEEE Photonics Soc. Summer Top. Meet. Ser. PHOSST 2010, vol. 18, no. 11, pp. 197-198, 2010.

[14] P. Burger, M. Keters, O. Evrard, and L. Van Buul, "Industrial silicon detectors, advancements in planar technology," Nucl. Instruments Methods Phys. Res. Sect. A Accel. Spectrometers, Detect. Assoc. Equip., vol. 591, no. 1, pp. 1-5, 2008.

[15] S. Member, D. R. Franklin, M. L. F. Lerch, M. Petasecca, G. U. Pignatel, M. Reinhard, G. D. Betta, S. Member, N. Zorzi, A. B. Rosenfeld, and S. Member, "Evaluation of Silicon Detectors With Integrated JFET for Biomedical Applications," vol. 56, no. 3, pp. 1051-1055, 2009.

[16] H. Rong, S. Xu, Y.-H. Kuo, V. Sih, O. Cohen, O. Raday, and M. Paniccia, "Lowthreshold continuous-wave Raman silicon laser," Nat. Photonics, vol. 1, no. 4, pp. 
232-237, 2007.

[17] V. Raghunathan, D. Borlaug, R. R. Rice, and B. Jalali, "Demonstration of a Midinfrared silicon Raman amplifier.," Opt. Express, vol. 15, no. 22, pp. 14355-62, 2007.

[18] K. Tiefenthaler and W. Lukosz, "Sensitivity of grating couplers as integratedoptical chemical sensors," vol. 6, no. 2, pp. 209-220, 1989.

[19] A. Manz, H. M. Widmers, and N. Graber, "Miniaturized total chemical analysis systems: A novel concept for chemical sensing," Sensors Actuators B Chem., vol. 1, no. 1-6, pp. 244-248, 1990.

[20] P. Neuži, S. Giselbrecht, K. Länge, T. J. Huang, and A. Manz, "Revisiting lab-ona-chip technology for drug discovery.," Nat. Rev. Drug Discov., vol. 11, no. 8, pp. 620-32, 2012.

[21] N. Fabricius, G. Gauglitz, and J. Ingenhoff, "A gas sensor based on an integrated optical Mach-Zehnder interferometer," vol. 7, pp. 672-676, 1992.

[22] T. Koster and P. Lambeck, "An integrated optical platform for absorptive sensing of chemical concentrations using chemo-optical monolayers," Meas. Sci. Technol., vol. 13, no. 8, pp. 1230-1238, 2002.

[23] G. Gauglitz and J. Ingenhoff, "Hydrocarbons,” vol. 11, pp. 207-212, 1993.

[24] C. Y. Chao and L. J. Guo, "Biochemical sensors based on polymer microrings with sharp asymmetrical resonance," Appl. Phys. Lett., vol. 83, no. 8, pp. 1527-1529, 2003.

[25] T. M. Butler, E. Igata, S. J. Sheard, and N. Blackie, "Integrated optical Bragggrating-based chemical sensor on a curved input edge waveguide structure.," Opt. 
Lett., vol. 24, no. 8, pp. 525-7, 1999.

[26] W. Lukosz and K. Tiefenthaler, "Sensitivity of integrated couplers as (bio)chemical," Sensors (Peterborough, NH), vol. 15, pp. 273-284, 1988.

[27] A. Densmore, D. X. Xu, P. Waldron, S. Janz, P. Cheben, J. Lapointe, a. Delâge, B. Lamontagne, J. H. Schmid, and E. Post, “A silicon-on-insulator photonic wire based evanescent field sensor," IEEE Photonics Technol. Lett., vol. 18, no. 23, pp. $2520-2522,2006$.

[28] T. Claes, J. G. Molera, K. De Vos, E. Schacht, R. Baets, and P. Bienstman, "Labelfree biosensing with a slot-waveguide-based ring resonator in silicon on insulator," IEEE Photonics J., vol. 1, no. 3, pp. 197-204, 2009.

[29] V. M. N. Passaro, F. Dell'Olio, C. Ciminelli, and M. N. Armenise, "Efficient chemical sensing by coupled slot SOI waveguides," Sensors, vol. 9, pp. 10121032, 2009.

[30] T. Kamalakis and T. Sphicopoulos, "Numerical study of the fabrication tolerance of conventional and MMI-flattened AWGs," IEEE Photonics Technology Letters, vol. 16, no. 8. pp. 1876-1878, 2004.

[31] D. X. Xu, a Densmore, a Delâge, P. Waldron, R. McKinnon, S. Janz, J. Lapointe, G. Lopinski, T. Mischki, E. Post, P. Cheben, and J. H. Schmid, "Folded cavity SOI microring sensors for high sensitivity and real time measurement of biomolecular binding.," Opt. Express, vol. 16, no. 19, pp. 15137-15148, 2008.

[32] D. Dai and S. He, "Mach - Zehnder interferometer-coupled microring," vol. 26, no. 3, pp. 511-516, 2009.

[33] J. Wang and D. Dai, "Highly sensitive Si nanowire-based optical sensor using a 
Mach-Zehnder interferometer coupled microring.," Opt. Lett., vol. 35, no. 24, pp. 4229-31, 2010.

[34] W. Lukosz, "Principles and sensitivities of integrated optical and surface plasmon sensors for direct affinity sensing and immunosensing," Biosens. Bioelectron., vol. 6, no. 3, pp. 215-225, 1991.

[35] D. Clerc and W. Lukosz, "Integrated optical output grating coupler as refractometer and ( bio- ) chemical sensor," vol. 19, pp. 461-465, 1993.

[36] C. Stamm and W. Lukosz, "Integrated optical difference interferometer as immunosensor," Sensors Actuators, B Chem., vol. 31, no. 3, pp. 203-207, 1996.

[37] P. V. Lambeck, "Remote opto-chemical sensing with extreme sensitivity: Design, fabrication and performance of a pigtailed integrated optical phase-modulated Mach-Zehnder interferometer system," Sensors Actuators, B Chem., vol. 61, no. 1, pp. 100-127, 1999.

[38] A. Brandenburg, "Differential refractometry by an integrated-optical Young interferometer," Sensors Actuators B Chem., vol. 39, no. 1-3, pp. 266-271, 1997.

[39] E. Krioukov, D. J. W. Klunder, a Driessen, J. Greve, and C. Otto, "Sensor based on an integrated optical microcavity.," Opt. Lett., vol. 27, no. 7, pp. 512-514, 2002.

[40] a Ksendzov and Y. Lin, "Integrated optics ring-resonator sensors for protein detection.," Opt. Lett., vol. 30, no. 24, pp. 3344-3346, 2005.

[41] G. J. Veldhuis, O. Parriaux, H. J. W. M. Hoekstra, and P. V. Lambeck, "Sensitivity Enhancement in Evanescent Optical Waveguide Sensors," J. Light. Technol., vol. 18, no. 5, pp. 677-682, 2000. 
[42] O. Parriaux and G. J. Veldhuis, "Normalized analysis for the sensitivity optimization of integrated optical evanescent-wave sensors," J. Light. Technol., vol. 16, no. 4, pp. 573-582, 1998.

[43] K. De Vos, I. Bartolozzi, E. Schacht, P. Bienstman, and R. Baets, "Silicon-onInsulator microring resonator for sensitive and label-free biosensing.," Opt. Express, vol. 15, no. 12, pp. 7610-7615, 2007.

[44] C. A. Barrios, K. B. Gylfason, B. Sánchez, A. Griol, H. Sohlström, M. Holgado, and R. Casquel, "Bar07-Ol,” vol. 32, no. 21, pp. 3080-3082, 2007.

[45] V. R. Almeida, Q. Xu, C. A. Barrios, and M. Lipson, "Guiding and confining light in void nanostructure," Opt. Lett., vol. 29, no. 11, pp. 1209-1211, 2004.

[46] W. Green, R. Lee, G. Derose, A. Scherer, and A. Yariv, "Hybrid InGaAsP-InP Mach-Zehnder Racetrack Resonator for Thermooptic Switching and Coupling Control.," Opt. Express, vol. 13, no. 5, pp. 1651-1659, 2005.

[47] L. Zhou and A. W. Poon, "Electrically reconfigurable silicon microring resonatorbased filter with waveguide-coupled feedback.," Opt. Express, vol. 15, no. 15, pp. 9194-9204, 2007.

[48] A. Yariv and P. Yeh, Photonics: Optical Electronics in Modern Communications. Oxford University Press, 2007.

[49] A. P. K. Graham T. Reed, Silicon Photonics: An Introduction. London: Wiley, 2004.

[50] A. Yariv, "Coupled-Mode theory for guided-wave optics," IEEE J. Quantum Electron., vol. 9, no. 9, pp. 919-933, 1973.

[51] W. Streifer, M. Osinski, and A. Hardy, "Reformulation of the coupled-mode 
theory of multiwaveguide systems," J. Light. Technol., vol. 5, pp. 1-4, 1987.

[52] H. Kogelnik, "Theory of dielectric waveguides," Integr. Opt., pp. 13-81, 1975.

[53] O. Bryngdahl, "Image formation using self-imaging techniques," J. Opt. Soc. Am., vol. 63 , no. 4, p. 416, 1973.

[54] R. Ulrich, “Nc .;,, ,":,..4;. : \% : ..,” vol. 13, no. 3, pp. 259-264, 1975.

[55] W. Bogaerts, P. de Heyn, T. van Vaerenbergh, K. de Vos, S. Kumar Selvaraja, T. Claes, P. Dumon, P. Bienstman, D. van Thourhout, and R. Baets, "Silicon microring resonators," Laser Photonics Rev., vol. 6, no. 1, pp. 47-73, 2012.

[56] Y. Xiong and W. N. Ye, "Slotted silicon microring resonators with multimode interferometer couplers," IEEE Int. Conf. Gr. IV Photonics GFP, vol. 7, pp. 118$119,2013$.

[57] H. Kogelnik, "2 . Theory of Optical Waveguides," in Guided Wave OptoElectronics T. Tamir, 2nd ed., Berlin, Germany: Springer-Verlag, 1988, pp. 7-87.

[58] Y. Xiong and W. N. Ye, "Silicon Mach-Zehnder interferometer racetrack microring for sensing,” vol. 8990, p. 89901H, Mar. 2014.

[59] D.-X. Xu, A. Densmore, P. Waldron, J. Lapointe, E. Post, A. Delâge, S. Janz, P. Cheben, J. H. Schmid, and B. Lamontagne, "High bandwidth SOI photonic wire ring resonators using MMI couplers.," Opt. Express, vol. 15, no. 6, pp. 3149-3155, 2007.

[60] L. Shaped, E. Face, P. E. Face, and P. M. Dispersion, “Applications : Product Description :," pp. 1-7.

[61] V. R. Almeida, R. R. Panepucci, and M. Lipson, "Nanotaper for compact mode conversion.," Opt. Lett., vol. 28, no. 15, pp. 1302-1304, 2003. 
[62] G. Ren, S. Chen, Y. Cheng, and Y. Zhai, "Study on inverse taper based mode transformer for low loss coupling between silicon wire waveguide and lensed fiber," Opt. Commun., vol. 284, no. 19, pp. 4782-4788, 2011.

[63] M. Hammer and O. V. Ivanova, "Effective index approximations of photonic crystal slabs: A 2-to-1-D assessment," Opt. Quantum Electron., vol. 41, no. 4, pp. 267-283, 2009.

[64] A. W. Snyder and J. D. (John D. . Love, Optical waveguide theory. London; New York : Chapman and Hall, 1983.

[65] Lumerical, "Lumerical's 2.5D FDTD Propagation Method." [Online]. Available: https://www.lumerical.com/support/whitepaper/2.5d_fdtd_propagation_method.ht $\mathrm{ml}$.

[66] W. M. Haynes, "No Title," in CRC Handbook of Chemistry and Physics, 2013, pp. $145-146$.

[67] I. M. White and X. Fan, "On the performance quantification of resonant refractive index sensors.," Opt. Express, vol. 16, no. 2, pp. 1020-1028, Jan. 2008.

[68] M. Sumetsky, "Optimization of optical ring resonator devices for sensing applications.," Opt. Lett., vol. 32, no. 17, pp. 2577-9, 2007.

[69] M. Terrel, M. J. F. Digonnet, and S. Fan, "Ring-coupled Mach-Zehnder interferometer optimized for sensing.," Appl. Opt., vol. 48, pp. 4874-4879, 2009. 